Ann. Scient. Éc. Norm. Sup.,

$4^{\mathrm{e}}$ série, t. 40, 2007, p. 209 à 249.

\title{
ACTIONS OF LARGE SEMIGROUPS AND RANDOM WALKS ON ISOMETRIC EXTENSIONS OF BOUNDARIES
}

\author{
By Yves GUIVARC'H AND Albert RAUGi
}

ABSTRACT. - Let $G$ be a real algebraic semi-simple group, $X$ an isometric extension of the flag space of $G$ by a compact group $C$. We assume that $G$ is topologically transitive on $X$. We consider a closed sub-semigroup $T$ of $G$ and a probability measure $\mu$ on $T$ such that $T$ is Zariski-dense in $G$ and the support of $\mu$ generates $T$. We show that there is a finite number of $T$-invariant minimal subsets in $X$ and these minimal subsets are the supports of the extremal $\mu$-stationary measures on $X$. We describe the structure of these measures, we show the conditional equidistribution on $C$ of the $\mu$-random walk and we calculate the algebraic hull of the corresponding cocycle. A certain subgroup generated by the "spectrum" of $T$ can be calculated and plays an essential role in the proofs.

(c) 2007 Published by Elsevier Masson SAS

RÉSUMÉ. - Soient $G$ un groupe semi-simple algébrique réel, $X$ une extension isométrique de l'espace des drapeaux de $G$ par un groupe compact. Nous supposons que $G$ est topologiquement transitif sur $X$. Nous considérons un sous-semi-groupe fermé $T$ de $G$, Zariski-dense, et une mesure de probabilité $\mu$ sur $T$ adaptée à $T$. Nous montrons que l'ensemble des fermés $T$-invariants minimaux de $X$ est fini et que ces fermés minimaux sont les supports des mesures de probabilités $\mu$-stationnaires extrémales. Nous décrivons la structure de ces probabilités, nous montrons l'équidistribution conditionnelle de la marche aléatoire de loi $\mu$ et nous déterminons l'enveloppe algébrique du cocycle associé. Un certain sous-groupe engendré par le «spectre» de $T$ est calculé et joue un rôle essentiel dans les démonstrations.

(C) 2007 Published by Elsevier Masson SAS

\section{Introduction}

Let $\mathbf{G}$ be a real algebraic semi-simple group, $G=\mathbf{G}(\mathbb{R})$ the group of its real points. We assume $\mathbf{G}$ to be Zariski-connected and we consider an Iwasawa decomposition $G=K A N^{+}$ where $K$ (resp. $A, N^{+}$) is a maximal compact (resp. a polar, an unipotent) subgroup. We denote by $M$ the centralizer of $A$ in $K$, so that $F=G / M A N^{+}$is the maximal flag space of $G$, and we call $\pi$ the natural map from $G$ onto $F$. Let $A^{+} \subset A$ be the open Weyl chamber in $A$ defined by $N^{+}$. We say that $g \in G$ is flag-proximal ( $F$-proximal) if $g$ is conjugate to an element $\tilde{g} \in M A^{+}$; in this case $g$ is semi-simple and the action of $g$ on $F$ has a unique attractive fixed point $g^{-}$ in $F$. We observe that $\tilde{g}$ is uniquely defined modulo conjugacy by $M$. For a subset $E$ of $G$, we denote by $E^{\text {prox }}$ the subset of its proximal elements. Then, for $g \in G^{\text {prox }}$ we can define $\lambda(g) \in M A /[M, M]$ to be the projection of $\tilde{g}$ on $M A /[M, M]$.

We consider a Zariski-dense sub-semigroup $T$ of $G$ and we denote by $\langle\operatorname{Spec} T\rangle$ the closed subgroup of $M A /[M, M]$ generated by $\left\{\lambda(t): t \in T^{\text {prox }}\right\}$. It is well-known (see [6] Appendix or [26]) that if $T$ is Zariski-dense then $T^{\text {prox }} \neq \emptyset$. Then one of our main results is the following theorem. 
THEOREM 1. - Consider a Zariski-dense sub-semigroup $T$ of $G$. Then the closed subgroup $\langle\operatorname{Spec} T\rangle$ has finite index in $M A /[M, M]$.

As a consequence, we recover a previous result of [4]: the projection of $\langle\operatorname{Spec} T\rangle$ in $A$ is equal to $A$. For example, if we take $G=\operatorname{SL}(d, \mathbb{C})$, then $A=\left(\mathbb{R}_{+}^{*}\right)^{d-1}, M=(\mathbb{T})^{d-1}$ and $M A=\left(\mathbb{C}^{*}\right)^{d-1}$. In this case we obtain $\langle\operatorname{Spec} T\rangle=M A$.

We recall (see [16]) that the limit set $L_{T} \subset F$ of $T$ is defined as $L_{T}=\operatorname{closure}\left\{t^{-}: t \in T^{\text {prox }}\right\}$ and $L_{T}$ is the unique $T$-invariant minimal closed subset of $F$. Let $C$ be a compact metric group, $\rho$ a continuous homomorphism of $M A$ into $C$ such that $\rho(M A)$ is dense in $C$. We consider the skew product $X^{\rho}$ of $F$ by $C$, associated with the equivalence relation

$$
\forall g \in G, \forall z \in M A, \forall u \in N^{+}, \quad(g z u, c) \sim(g, \rho(z) c) .
$$

We observe that $X^{\rho}$ is a compact $G$-space which is a trivial $C$-bundle over $F$ with typical fiber $C$. In particular, the homogeneous space $X^{\rho}=G / D N^{+}$, where $D$ is a cocompact normal subgroup of $A M$, is an example of such skew product with $C=A M / D$ and $\rho$ the projection of $A M$ onto $A M / D$. We denote by $L_{T}^{\rho}$ the inverse image of $L_{T}$ in $X^{\rho}$ and we remark that $L_{T}^{\rho}$ is a $T$-invariant closed subset of $X^{\rho}$. Let $H_{T}$ be the inverse image of $\langle\operatorname{Spec} T\rangle$ in $M A$, we observe that, from Theorem 1 we can write $H_{T}=A M_{T}$ where $M_{T}$ is a subgroup of finite index in $M$. It follows that $\overline{\rho\left(A M_{T}\right)}=C_{T}$ is a normal subgroup of finite index in $C$ and $C / C_{T}=\rho(M) / \rho\left(M_{T}\right)$ is abelian. Then the structure of $T$-orbit closures in $X^{\rho}$ is given by the following theorem.

THEOREM 2. - The $G$-space $X^{\rho}$ has a finite number of $T$-invariant minimal closed subsets $X_{c}^{\rho}, c \in C / C_{T}$. If we choose a point $x_{\rho} \in L_{T}^{\rho}$, then each of them is $C_{T}$-right invariant and can be written as $X_{c}^{\rho}=\overline{T \cdot x_{\rho}} c$ with $c \in C / C_{T}$. Furthermore $L_{T}^{\rho}$ is the disjoint union of the $X_{c}^{\rho}$, $c \in C / C_{T}$.

For $G=\operatorname{SL}(d, \mathbb{C})$, we have always $C_{T}=C$ and consequently, for any $x \in X^{\rho}, \overline{T \cdot x} \supset L_{T}^{\rho}$.

The $T$-orbit closures on $X^{\rho}$ are closely related to stationary measures for random walks on $X^{\rho}$. Let $\mu$ be a probability measure on $G$ such that the closed sub-semigroup generated by its support is equal to $T$. We consider on $X^{\rho}$ the Markov operator $P_{\mu}$ defined by, for any bounded Borel function $f$ on $X^{\rho}$,

$$
\forall x \in X^{\rho}, \quad P_{\mu} f(x)=\int_{X^{\rho}} f(g \cdot x) \mu(d g) .
$$

A probability $\sigma$ on the $G$-space $X^{\rho}$ is said to be $\mu$-stationary (or $\mu$-invariant, or $P_{\mu}$-invariant) if $\mu * \sigma=\sigma$ (or, for any bounded Borel function $f, \sigma\left(P_{\mu} f\right)=\sigma(f)$ ).

THEOREM 3.-For any continuous function $f$ on $X^{\rho}$, the family $\left\{P_{\mu}^{n} f: n \in \mathbb{N}\right\}$ is equicontinuous. The operator $P_{\mu}$ has a finite number of extremal invariant probabilities $\sigma_{c}$, $c \in C / C_{T}$. Each of them is $C_{T}$-right invariant and $\sigma_{c}=\sigma_{e} * \delta_{c}, c \in C / C_{T}$, has support equal to $X_{c}^{\rho}$. Furthermore, the peripheral spectrum of $P_{\mu}$ is either $\{1\}$ or $\{-1,1\}$. In the first (resp. last) case, for any continuous function $f$, the sequence $P_{\mu}^{n} f$ (resp. $P_{\mu}^{2 n} f$ ) converges uniformly on $X^{\rho}$. When $C$ is connected, we are in the first case.

We consider the product space $\Omega=G^{\mathbb{Z}}$ endowed with the shift $\theta$ and the product probability $\mathbb{P}=\mu \otimes \mathbb{Z}$. We denote by $Y_{k}, k \in \mathbb{Z}$, the coordinate maps of $\Omega$. Setting

$$
S_{0}=e ; \quad \forall n \in \mathbb{N}^{*}, \quad S_{n}=Y_{0} \cdots Y_{n-1} \quad \text { and } \quad S_{-n}=Y_{-1}^{-1} \cdots Y_{-n}^{-1},
$$

$4^{\text {e }}$ SÉRIE - TOME $40-2007-\mathrm{N}^{\circ} 2$ 
we obtain a $G$-valued $\mathbb{Z}$-cocycle (i.e. $\forall n, p \in \mathbb{Z}, S_{n+p}=S_{n} S_{p} \circ \theta^{n}$ ). Then we obtain the following result.

THEOREM 4. - The cocycle defined by the random walk $S_{n}, n \in \mathbb{Z}$, is cohomologous to an $A M_{T}$-valued cocycle. The subgroup $A M_{T}$ is the smallest closed subgroup of $G$ which has such a property (up to conjugacy). As a consequence, the algebraic hull (see [35]) of the cocycle $S_{n}$ is equal to $A M$.

In Sections 1 and 2 we describe the main results and notations. The proofs are given in Sections 3 and 4 . The study of the group $\langle\operatorname{Spec} T\rangle$ is motivated by various problems, some of them are considered in Section 5 below. Among them we mention:

- The asymptotic behavior of products of random matrices (for example complex matrices) when the Lyapunov spectrum is not simple (see $[2,10,18]$ ).

- The classification of convex divisible cones (see [5]).

- The dynamics of sub-semigroups of $M(n, \mathbb{Z})$ acting on the torus $\mathbb{T}^{n}$ (see [20,23]).

- The mixing properties of geodesic frame flows in constant negative curvature (see [22]).

- The renewal theorem for products of random matrices (see [19]).

Finally we consider examples of classical groups which show the geometric implications of the various possibilities for the groups $M_{T}, M, M A, M A /[M, M], M / M_{0}$.

In complement by S. Cantat (Section 6), some implications for the local structure of the limit sets of $T$ are considered when $G=\operatorname{SL}(n, \mathbb{C})$. In complement by G. Prasad (Section 7), Diophantine properties of elements of $T$ are discussed.

\section{Spectrum and limit sets of a sub-semigroup of a semi-simple Lie group}

\subsection{Decompositions of semi-simple real Lie group}

We consider a Zariski-connected semi-simple $\mathbb{R}$-algebraic group $\mathbf{G} \subset \mathrm{GL}(d, \mathbb{C})$, i.e. a subgroup given as the set of zeros of a family of polynomials with real coefficients and we denote by $G=\mathbf{G}(\mathbb{R})$ the real points of $\mathbf{G}$. Such a group $G$ has always a finite number of connected components for the usual topology and a finite center. Any connected semi-simple subgroup of $\mathrm{GL}(n, \mathbb{R})$ is the connected component of a group of the form $G=\mathbf{G}(\mathbb{R})$ where $\mathbf{G}$ is Zariski connected (see [25]).

By abuse of language, we will say also that $\mathbf{G}(\mathbb{R})$ is an algebraic $\mathbb{R}$-group. We will make use of the Zariski topology on $G$; in particular for $Y \subset G$, we note $\mathcal{Z}(Y)$ the Zariski closure of $Y$ in $G$.

We call $e$ the unit element of $G,(\mathfrak{g},[]$,$) the Lie algebra of G$ and ad the adjoint representation of $\mathfrak{g}$ (i.e. for any $(X, Y) \in \mathcal{G}^{2}$, ad $\left.X(Y)=[X, Y]\right)$. Let $\mathfrak{g}=\mathfrak{k} \oplus \mathfrak{p}$ be a Cartan decomposition of $\mathfrak{g}$ and $\mathfrak{a}$ be a maximal abelian subalgebra of $\mathfrak{p}$. We call $\Delta$ the set of roots of ad $\mathfrak{a}$ and for any $\alpha \in \Delta$, we call $\mathfrak{g}_{\alpha}$ the root space associated with $\alpha$,

$$
\mathfrak{g}_{\alpha}=\{X \in \mathfrak{g}: \forall H \in \mathfrak{a}, \operatorname{ad} H(X)=\alpha(H) X\} .
$$

We have $\mathfrak{g}=\bigoplus_{\alpha \in \Delta} \mathfrak{g}_{\alpha}$ and $\mathfrak{g}_{0}=\mathfrak{a} \oplus \mathfrak{m}$, where $\mathfrak{m}=\{X \in \mathfrak{k}:[X, \mathfrak{a}]=0\}$. We choose an open Weyl chamber $\mathfrak{a}^{+}$in $\mathfrak{a}$ and we call $\Delta_{+}$[resp. $\Delta_{-}$] the set of positive [resp. negative] roots on $\mathfrak{a}^{+}$. Setting

$$
\mathfrak{n}^{+}=\bigoplus_{\alpha \in \Delta_{+}} \mathfrak{g}_{\alpha} \quad \text { and } \quad \mathfrak{n}^{-}=\bigoplus_{\alpha \in \Delta_{-}} \mathfrak{g}_{\alpha}
$$


we obtain two nilpotent subalgebras of $\mathfrak{g}$ normalized by $\mathfrak{a}$ and the following decompositions for $\mathfrak{g}$ : the Iwasawa decompositions

$$
\mathfrak{g}=\mathfrak{n}^{-} \oplus \mathfrak{a} \oplus \mathfrak{k} \quad \text { and } \quad \mathfrak{g}=\mathfrak{k} \oplus \mathfrak{a} \oplus \mathfrak{n}^{+}
$$

and the Bruhat decomposition

$$
\mathfrak{g}=\mathfrak{n}^{-} \oplus \mathfrak{a} \oplus \mathfrak{m} \oplus \mathfrak{n}^{+} .
$$

Let us call $N^{+}, N^{-}$and $A$ the connected Lie subgroups of $G$ having respectively $\mathfrak{n}^{+}, \mathfrak{n}^{-}$and $\mathfrak{a}$ for Lie algebras. We denote by $K$ the maximal compact subgroup of $G$ whose Lie algebra is $\mathfrak{k}$ and by $M$ the centralizer of $A$ in $K$. Then the centralizer of $A$ in $G$ is $M A, M \cap A=\{e\}$, and the subgroup $P^{+}=M A N^{+}$is a so-called minimal standard parabolic subgroup of $G$. We set $A^{+}=\operatorname{expa}^{+}$. Then we obtain for $G$ the following decompositions.

Iwasawa decompositions: $G=N^{-} A K$ [resp. $G=K A N^{+}$]; the map $(n, a, k) \rightarrow n a k$ is an analytic isomorphism from $N^{-} \times A \times K$ onto $G$. For any $g \in G$, we write $g=\mathbf{u}(g) \mathbf{b}(g) \mathbf{k}(g)$ [resp. $g=\mathbf{k}^{\prime}(g) \mathbf{b}^{\prime}(g) \mathbf{u}^{\prime}(g)$ ] the Iwasawa decomposition of $g$.

Polar decomposition: $G=K \overline{A^{+}} K$; any $g \in G$ is written $g=\eta \mathbf{a}(g) \kappa$ where $\eta, \kappa \in K$ and $\mathbf{a}(g) \in \overline{A^{+}}$; the component $\mathbf{a}(g)$ is unique, the pair $(\eta, \kappa)$ can be replaced by $\left(\eta m, m^{-1} \kappa\right)$ for any $m \in K$ commuting with $\mathbf{a}(g)$.

Bruhat decomposition: Let $M^{\prime}$ be the normalizer of $A$ in $K$. We have $G=N^{-} M^{\prime} A N^{+}$. Denoting by $W$ a complete set of coset representatives of the quotient group $M^{\prime} / M$, we obtain $G=N^{-} W M A N^{+}$. The map $\left(v, a, m, v^{\prime}\right) \rightarrow v a m v^{\prime}$ is an algebraic isomorphism from the product space $B=N^{-} \times A \times M \times N^{+}$onto the Zariski-dense open set $N^{-} A M N^{+}$in $G$. For $g \in N^{-} A M N^{+}$we call $\zeta(g)$ and $\eta(g)$ the components of $g$ on $N^{-}$and $N^{+}$. We note $[M, M]$ the commutator subgroup of $M$.

We denote by Ad the adjoint representation of $G$ on $\mathfrak{g}$. For any $\alpha \in \Delta$, we call $\varphi_{\alpha}$ the homomorphism of $A$ in $\left(\mathbb{R}_{+}^{*}, \times\right)$ defined by: $\forall H \in \mathfrak{a}, \varphi_{\alpha}(\exp H)=e^{\alpha(H)}$.

We call $\pi$ [resp. $\pi^{\prime}$ ] the natural map from $G$ onto the boundary $F=G / M A N^{+}$[resp. $F^{\prime}=N^{-} M A \backslash G$ ] of $G$; we write $g \cdot x$ [resp. $x^{\prime} \cdot g$ ] the action of $g \in G$ on $x \in F$ [resp. on $x^{\prime} \in F^{\prime}$. For any element $x \in \pi\left(N^{-}\right)$, we denote by $\hat{x}$ the unique element of $N^{-}$such that $\pi(\hat{x})=x$.

Remark. - The results of this paper remain valid, up to some modifications, if $G$ is a semisimple real Lie group with a finite center and a finite number of connected components. In this more general situation, the maximal compact subgroup $K$ meets each connected component of $G$ and the subgroup $M$ has to be replaced by the normalizer $M^{1}$ in $K$ of $A$ and $N^{+}$ (see [31], Proposition (1.7)). Then the intersection of $M^{1}$ with the unit component $G_{0}$ of $G$ is the centralizer of $A$ in $K \cap G_{0}$. Furthermore, $F=G / M^{1} A N^{+}$can be shown to be the largest compact $G$-space on which the action of $G$ is minimal and proximal, hence is a $G$-boundary in the sense of Furstenberg [14]. When $M_{1}=M$, the cell $N^{-} M A N$ is dense (usual topology) in $G$.

1.1. Hypothesis $(\mathrm{H})$. - We say that a sub-semigroup $T$ of $G$ satisfies hypothesis $(\mathrm{H})$ if it satisfies the two following assumptions:

(i) there does not exist a finite number of elements of $G, g_{0}, \ldots, g_{r}, r \in \mathbb{N}^{*}$, such that

$$
T g_{0} \subset \bigcup_{1 \leqslant k \leqslant r} g_{i}\left(N^{-} M A N^{+}\right)^{c} ;
$$

(ii) there exists an element $g$ in $T$ which is conjugate to an element of $M A^{+}$. Under condition (i) we can replace (ii) by the condition (see [16]):

$4^{\text {e }}$ SÉRIE - TOME $40-2007-\mathrm{N}^{\circ} 2$ 
(ii') for any $\alpha \in \Delta_{+}$, there exists a $T$-valued sequence $\left(g_{n}\right)_{n \geqslant 0}$ such that $\varphi_{\alpha}\left(a\left(g_{n}\right)\right) \rightarrow+\infty$, when $n \rightarrow+\infty$.

When $G$ is a Zariski connected algebraic real semi-simple group and the Zariski closure of $T$ is equal to $G$, the hypothesis (H) is satisfied ([26], [6] Appendix).

By extension, we say that a probability measure $\mu$ on the Borel $\sigma$-algebra $\mathcal{B}(G)$ of $G$ satisfies hypothesis $(\mathrm{H})$ if the closed sub-semigroup $T_{\mu}$ of $G$ generated by the $\operatorname{support} \operatorname{Supp}(\mu)$ of $\mu$ satisfies hypothesis $(\mathrm{H})$.

1.2. LEMMA (See $[14,17]$.$) - - For any probability measure \mu$ on $G$ satisfying hypothesis $(\mathrm{H})$, there exists a unique $\mu$-invariant probability measure $\nu$ on $F\left[\right.$ resp. $\nu^{\prime}$ on $\left.F^{\prime}\right]$. Furthermore $\nu$ [resp. $\left.\nu^{\prime}\right]$ is irreducible, i.e. $\forall g \in G, \nu\left(g \cdot \pi\left(N^{-}\right)\right)=1\left(\right.$ resp. $\left.\nu^{\prime}\left(\pi^{\prime}\left(N^{+}\right) \cdot g\right)=1\right)$.

\subsection{Spectrum and limit sets of a sub-semigroup of $G$}

An element $g \in G$ is said to be F-proximal if it is conjugate to an element of $\mathrm{MA}^{+}$; then we know that this element of $M A^{+}$is unique modulo $[M, M]$ and we denote by $\lambda(g)$ (resp. $\lambda^{u}(g)$ ) its projection on $M A /[M, M]$ (resp. $M /[M, M]$ ). We observe that, for any $y \in G$, we have $\lambda\left(y g y^{-1}\right)=\lambda(g)$.

For a sub-semigroup $T$ of $G$, we denote by $T^{\text {prox }}$ the set of $F$-proximal elements in $T$. The set $\operatorname{Spec} T=\left\{\lambda(t): t \in T^{\text {prox }}\right\}$ (resp. $\operatorname{Arg} T=\left\{\lambda^{u}(t): t \in T^{\text {prox }}\right\}$ ) is called the spectrum (resp. unit spectrum) of $T$. We denote by $\langle\operatorname{Spec} T\rangle(\operatorname{resp} .\langle\operatorname{Arg} T\rangle)$ the closed subgroup of $M A /[M, M]$ (resp. $M /[M, M]$ ), generated by $\operatorname{Spec} T$ (resp. $\operatorname{Arg} T$ ).

Let $g \in G^{\text {prox }}$, we can write $g=y_{a m y}{ }^{-1}$ with $y \in G$ and $a m \in M A^{+}$. For any $x \in y \cdot \pi\left(N^{-}\right)$, we have $\lim _{n} g^{n} \cdot x=g^{-}=\pi(y)$ and $g^{-}$is the unique attractive point of $g$. In addition, if $g \in G$ and $h \in G^{\text {prox }}$, for $n$ large enough $g h^{n} \in G^{\text {prox }}$ and $g \cdot h^{-}$is its unique attractive point (see [1]). The set $L_{T}=\operatorname{closure}\left\{t^{-}: t \in T^{\text {prox }}\right\}$ is called the limit set of $T$ (see [16]). It follows that $L_{T}$ is $T$-invariant; i.e. for any $(t, x) \in T \times L_{T}, t \cdot x$ belongs to $L_{T}$.

We observe that the word "proximal" is used here in a more general sense than the usual one, which is restricted to projective spaces and projective maps.

In the same way, replacing the left action of $G$ on $F$ by the right action of $G$ on $F^{\prime}$ we define the dual limit set $L_{T}^{\prime}=$ closure $\left\{t^{+}: t \in T^{\text {prox }}\right\}$. For any $g \in G^{\text {prox }}$, we can write $g=y a m y^{-1}$ with $y \in G$ and $a m \in M A^{+}$; then we have $g^{+}=\pi^{\prime}\left(y^{-1}\right)$. We can always assume that $\pi(e) \in L_{T}$ and $\pi^{\prime}(e) \in L_{T}^{\prime}$, by replacing $T$ by its conjugate $y^{-1} T y$.

Proposition 1.5 below gives us a characterization of the limit set and the dual limit set for a sub-semigroup $T$ which satisfies hypothesis $(\mathrm{H})$.

1.4. Definition. - Let $\mu$ be a probability measure on the Borel $\sigma$-algebra $\mathcal{B}(G)$ of $G$. Let us consider the product probability space $\Omega=G^{\mathbb{N}^{*}}$ endowed with the product measure $\mathbb{P}=\mu \bigotimes_{\mathbb{N}^{*}}$. We denote by $Y_{k}(\omega), k \in \mathbb{N}^{*}$, the coordinates of $\omega \in \Omega$ and by $\theta$ the shift transformation on $\Omega$, so that $Y_{k+1}(\omega)=Y_{1} \circ \theta^{k}(\omega)$. The shift $\theta$ preserves the probability measure $\mathbb{P}$ on $\Omega$ and $\mathbb{P}$ is $\theta$-ergodic. Furthermore, the $G$-valued random variables $Y_{k}, k \geqslant 1$ are independent and identically distributed with common law $\mu$. We set $R_{0}=L_{0}=e$ and for any $n \in \mathbb{N}^{*}$, we denote by $R_{n}$ the random product $Y_{1} \cdots Y_{n}$ and by $L_{n}$ the random product $Y_{n} \cdots Y_{1}$. The stochastic process $\left(L_{n}\right)_{n \geqslant 0}$ [resp. $\left(R_{n}\right)_{n \geqslant 0}$ ] is called the left [resp. right] random walk on $G$. A point $x \in F^{\prime}$ (resp. $x \in F)$ is said to be $\mu$-recurrent if, for any $r>0$,

$$
\sum_{n \in \mathbb{N}} 1_{B(x, r)}\left(x \cdot R_{n}\right)=+\infty \quad\left(\text { resp. } \sum_{n \in \mathbb{N}} 1_{B(x, r)}\left(L_{n} \cdot x\right)=+\infty\right), \quad \mathbb{P} \text {-a.e. }
$$


1.5. Proposition. - Let $T$ be a sub-semigroup of $G$ which satisfies hypothesis $(\mathrm{H})$. Then the limit set $L_{T}$ (resp. the dual limit set $L_{T}^{\prime}$ ) of $T$ is the unique minimal $T$-invariant subset of $F$ (resp. of $\left.F^{\prime}\right)$. For any probability measure $\mu$ on $G$ such that $T_{\mu}=T, L_{T}$ (resp. $\left.L_{T}^{\prime}\right)$ is the support of the unique $\mu$-invariant probability measure $\nu$ on $F$ (resp. $\nu^{\prime}$ on $\left.F^{\prime}\right)$. Moreover, $L_{T}$ (resp. $L_{T}^{\prime}$ ) is the set of $\mu$-recurrent points in $F$ (resp. in $F^{\prime}$ ). If $\pi(e) \in L_{T}$, and $P$ is any standard parabolic subgroup of $G$, there exists a sequence $\left(X_{n}\right)$ of elements of $\mathfrak{n}^{-}$such that

$$
\exp \left(X_{n}\right) \notin P, \quad \pi\left(\exp X_{n}\right) \in L_{T} \quad \text { and } \quad \lim _{n \rightarrow+\infty} X_{n}=0 .
$$

\section{6. $M A$-valued cocycles}

Let $g \in G$ and $x \in F$ be such that $x$ and $g \cdot x$ belong to $\pi\left(N^{-}\right)$. We define $\gamma(g, x)$ to be the $M A$-component of $g \hat{x}$ in the Bruhat decomposition:

$$
g \hat{x}=\widehat{g \cdot x} \gamma(g, x) n^{+}(g, x) \quad \text { with } n^{+}(g, x) \in N^{+} .
$$

For any $h \in G$, we define also: $\gamma_{h}(g, x)=\gamma\left(h g h^{-1}, h \cdot x\right)$, for any $(g, x) \in G \times F$ such that $h \cdot x$ and $h g \cdot x$ belong to $\pi\left(N^{-}\right)$; i.e. such that $x$ and $g \cdot x$ belong to $h^{-1} \cdot \pi\left(N^{-}\right)$.

We observe that, for any $h \in G, \gamma_{h}$ satisfies the cocycle relation:

$$
\gamma_{h}\left(g g^{\prime}, x\right)=\gamma_{h}\left(g, g^{\prime} \cdot x\right) \gamma_{h}\left(g^{\prime}, x\right)
$$

for any $\left(g, g^{\prime}, x\right) \in G \times G \times F$ such that $x, g^{\prime} \cdot x$ and $g g^{\prime} \cdot x$ belong to $h^{-1} \cdot \pi\left(N^{-}\right)$. When $h=n^{-} m a$ belongs to $N^{-} M A$, we have $\gamma(h, x)=m a$, for any $x \in \pi\left(N^{-}\right)$and $\gamma_{h}(g, x)=$ $m a \gamma(g, x)(m a)^{-1}=m \gamma(g, x) m^{-1}$, for any $(g, x) \in G \times F$ such that $x$ and $g \cdot x$ belong to $\pi\left(N^{-}\right)$.

We consider also the projection map pr of $M A$ onto the abelian group $M A /[M, M]$. It follows from the above that $\operatorname{pr}\left(\gamma_{h}\right)$ depends only on $\pi^{\prime}(h) \in F^{\prime}$.

1.7. Definition. - For any sub-semigroup $T$ of $G$, for any $g \in G$ and for $x \in L_{T} \cap g^{-1}$. $\pi\left(N^{-}\right)$, we denote by $\Gamma_{g, x}(T)$ the closed subgroup of $M A$ generated by the elements $\gamma_{g}(t, x)$, $t \in T$ such that $t \cdot x$ belong to $g^{-1} \cdot \pi\left(N^{-}\right)$. From the above, $\operatorname{pr}\left(\Gamma_{g, x}(T)\right)$ depends only on $\pi^{\prime}(g)$ and $x$. We call $\Gamma_{g}(T)$ the closed subgroup of $M A$ generated by $\Gamma_{g, x}(T)$ when $x$ varies in $L_{T} \cap g^{-1} \cdot \pi\left(N^{-}\right)$.

1.8. Proposition. - Let $T$ be a sub-semigroup of $G$ which satisfies hypothesis $(\mathrm{H})$. For any $g \in G$ and $x \in L_{T} \cap g^{-1} \cdot \pi\left(N^{-}\right)$, we have:

$$
\Gamma_{g, x}(T)=\Gamma_{g}(T), \quad \Gamma_{g}(T)=\Gamma_{e}\left(g T g^{-1}\right) \quad \text { and } \quad\langle\operatorname{Spec} T\rangle \subset \operatorname{pr}\left(\Gamma_{g}(T)\right) .
$$

For $g \in\left(\pi^{\prime}\right)^{-1}\left(L_{T}^{\prime}\right), \operatorname{pr}\left(\Gamma_{g}(T)\right)$ is independent of $g$ and $\langle\operatorname{Spec} T\rangle=\operatorname{pr}\left(\Gamma_{g}(T)\right)$.

1.9. THEOREM. - Let $G$ be an algebraic Zariski connected real semi-simple group and $T$ be a Zariski dense sub-semigroup of $G$. Then, for any $g \in G$, the group $\Gamma_{g}(T)$ is Zariski dense in $M A$ and contains the commutator subgroup $[M, M]$ of $M$.

For any $g \in\left(\pi^{\prime}\right)^{-1}\left(L_{T}^{\prime}\right)$, we have:

$$
\Gamma_{g}(T)=\operatorname{pr}^{-1}(\langle\operatorname{Spec} T\rangle)=A M_{T},
$$

where $M_{T}$ is a closed normal subgroup of $M$, containing the identity component $M_{0}$ of $M$. The quotient group $M_{T} / M_{0}=\operatorname{Sgn} T$ is a finite abelian group, isomorphic to $(\mathbb{Z} / 2 \mathbb{Z})^{p}$,

$4^{\text {e }}$ SÉRIE - TOME $40-2007-\mathrm{N}^{\circ} 2$ 
$0 \leqslant p \leqslant \operatorname{dim} A$. Moreover, for any $y \in G, M_{y T y^{-1}}=M_{T}$ and $M_{T^{-1}}=w M_{T} w^{-1}$, where $w$ is an element of the normalizer of $A$ in $K$ such that $\operatorname{Ad}(w)\left(\mathfrak{a}^{+}\right)=\mathfrak{a}^{-}$.

1.10. Corollary. $-\langle\operatorname{Arg} T\rangle$ is a subgroup of finite index in $M /[M, M]$ and $\langle\operatorname{Spec} T\rangle=$ $A\langle\operatorname{Arg} T\rangle$. The subgroup $M_{T}$ is the inverse image in $M$ of $\langle\operatorname{Arg} T\rangle$ and $\operatorname{Sgn} T=M_{T} / M_{0}=$ $\langle\operatorname{Arg} T\rangle /\langle\operatorname{Arg} T\rangle_{0}$.

\section{Random walks on isometric extensions of the boundary}

\section{- Skew product of the boundary with a compact group}

2.1. Let $\rho$ be a group homomorphism from $P^{+}=M A N^{+}$in a compact group $C$. We denote by $G \times{ }_{\rho} C$ the quotient of the product space $G \times C$ by the equivalence relation

$$
(g, c) \sim\left(g^{\prime}, c^{\prime}\right) \Longleftrightarrow \exists p \in P^{+}, \quad g=g^{\prime} p \quad \text { and } \quad c=\rho\left(p^{-1}\right) c^{\prime} .
$$

The group $G$ acts on $G \times{ }_{\rho} C$ in the following way:

$$
\forall h \in G, \quad h \cdot \overline{(g, c)}=\overline{(h g, c)} .
$$

For any $w \in W$, we denote by $N_{w}^{-}$the subgroup of $N^{-}$defined by

$$
N_{w}^{-}=N^{-} \cap\left(w^{-1} N^{-} w\right)=\exp \bigoplus_{\left\{\alpha \in \Delta_{-}: \alpha \circ A d w^{-1} \in \Delta_{-}\right\}} \mathfrak{g}_{\alpha}
$$

If we write $g=n^{-} w p^{+}$, with $w \in W, n^{-} \in N_{w}^{-}$and $p^{+} \in P^{+}$, we have $(g, c) \sim$ $\left(n^{-} w, \rho\left(p^{+}\right) c\right)$. Identifying $\bigcup_{w \in W} N_{w}^{-} w$ to $F=G / P^{+}, G \times{ }_{\rho} C$ can be abusively written $F \times{ }_{\rho} C$ and, for any $(h, g) \in G^{2}$ such that $g$ and $h g$ belong to $B$, we have

$$
\forall c \in C, \quad h \cdot(\pi(g), c)=(\pi(h g), \rho(\gamma(h, \pi(g))) c) .
$$

It follows that the map $(g, c) \longrightarrow \pi(g)$ defines a $G$-equivariant map from $G \times{ }_{\rho} C$ to $F$. We denote this map by $\pi_{\rho}$.

We denote by $C\left(F \times{ }_{\rho} C\right)$ the space of continuous functions on the skew product $F \times{ }_{\rho} C$. For any probability measure $\mu$ on $G$, we consider the Markov operator $P_{\mu}$ on $C\left(F \times{ }_{\rho} C\right)$ defined by:

$$
\forall(x, c) \in F \times{ }_{\rho} C, \quad P_{\mu} f(x, c)=\int_{G} f(g \cdot(x, c)) \mu(d g) .
$$

For any $u \in N^{+}$and $a \in A^{+}$, we have $a^{-n} u a_{n \rightarrow+\infty}^{n}$. Consequently, $\rho\left(N^{+}\right)=\{\rho(e)\}$. Even if it means to replace $C$ by a smaller closed subgroup, in what follows we assume that the closure $\overline{\rho(A M)}$ of $\rho(A M)$ is equal to $C$. Then every $G$-orbit in $X^{\rho}$ is dense. The inverse image of $N^{-} \cdot \pi(e)$ in $X^{\rho}$ is an open dense subset. For the measures, which occur here, we can identify $X^{\rho}$ with $\pi\left(N^{-}\right) \times C$. We will also write $\pi_{\rho}^{-1}\left(N^{-} \cdot \pi(e)\right)=\pi\left(N^{-}\right) \times C$.

\section{- Decomposition of $X^{\rho}=F \times{ }_{\rho} C$ and $\mu$-stationary measures}

2.2. LemMA. - We consider a decomposition of $C$ in $C_{T}$-classes: $C=\bigcup_{i=1}^{s} C_{T} c_{i}, c_{i} \in C$, $c_{1}=e$. We assume $\pi(e) \in L_{T}$ and we choose $e_{\rho} \in L_{T}^{\rho}$ with $\pi_{\rho}\left(e_{\rho}\right)=\pi(e)$. We denote by $\overline{X_{1}^{\rho}}$ the 
closure of $X_{1}^{\rho}=L_{T}^{\rho} \cap\left(N^{-} \cdot e_{\rho}\right) C_{T}$. Then $L_{T}^{\rho}$ is the disjoint union of the closed T-invariant sets $\overline{X_{i}^{\rho}}=\overline{X_{1}^{\rho}} c_{i}, 1 \leqslant i \leqslant s$.

Proof. - Clearly $\left(N^{-} \cdot e_{\rho}\right) C$ is a disjoint union of the open sets $\left(N^{-} \cdot e_{\rho}\right) C_{T} c_{i}, 1 \leqslant i \leqslant s$. It follows that $\overline{X_{1}^{\rho}} \backslash X_{1}^{\rho}$ is contained in $\pi_{\rho}^{-1}\left(F \backslash \pi\left(N^{-}\right)\right)$, hence $\overline{X_{i}^{\rho}} \cap \overline{X_{j}^{\rho}}$ is contained in $\pi_{\rho}^{-1}\left(F \backslash \pi\left(N^{-}\right)\right)$, if $i \neq j$. On the other hand, we see that $\overline{X_{1}^{\rho}}$ is $T$-invariant as follows. Assume that $x_{n}=\left(y_{n} \cdot e_{\rho}\right) c_{n} \in X_{1}^{\rho}, y_{n} \in N^{-}, c_{n} \in C_{T}$ converges to $x \in \overline{X_{1}^{\rho}}$. Let $t$ be a fixed element of $T$. Using Proposition 1.5, we can assume that $t y_{n} \in N^{-} M A N^{+}$. Then the definition of $M_{T}$ and $C_{T}$ shows that $t \cdot x_{n} \in X_{1}^{\rho}$, hence $t \cdot x \in \overline{X_{1}^{\rho}}$. It follows that $\pi_{\rho}\left(\overline{X_{i}^{\rho}} \cap \overline{X_{j}^{\rho}}\right)$ is a closed $T$-invariant subset of $F \backslash \pi\left(N^{-}\right)$. The hypothesis (H) for $T$ implies $\overline{X_{i}^{\rho}} \cap \overline{X_{j}^{\rho}}=\emptyset$, if $i \neq j$. Furthermore, the relation $\pi_{\rho}^{-1}\left(L_{T} \cap \pi\left(N^{-}\right)\right)=\bigcup_{i=1}^{s} X_{i}^{\rho}$ implies $L_{T}^{\rho}=\bigcup_{i=1}^{s} \overline{X_{i}^{\rho}}$. Hence the result.

We will show below (Corollary 2.5 ) that the sets $\overline{X_{i}^{\rho}}, 1 \leqslant i \leqslant s$, are the unique $T$-invariant minimal closed subsets of $X^{\rho}$. We denote by $\lambda$ the normalized Haar measure of $C_{T}$. We denote by $\hat{\nu}$ the image of the stationary measure $\nu$ by the map $x \rightarrow \hat{x}$ from $\pi\left(N^{-}\right)$to $N^{-}$. Then we see that the measures $\hat{\nu} \otimes \lambda * \delta_{c_{i}}$ on $X^{\rho}$ are $\mu$-stationary. Clearly their supports are the $\overline{X_{i}^{\rho}}, 1 \leqslant i \leqslant s$.

2.3. THEOREM. - Let $\mu$ be a probability measure on $G$ which satisfies hypothesis $(\mathrm{H})$. Then the Markov operator $P_{\mu}$ is equicontinuous, i.e. for any $f \in C\left(F \times{ }_{\rho} C\right)$, the family of functions $\left\{P_{\mu}^{n} f: n \in \mathbb{N}\right\}$ is equicontinuous.

By using the language of the general theory of the equicontinuous Markov operators (see [29, 32]), we show that there is a finite number of "minimal absorbing compact subsets" having all either one or two "cyclic class". When $X^{\rho}=F$, we know from [17] that the sequence of functions $\left(P^{n} f\right)_{n \geqslant 0}$ converges uniformly on $F$ to $\nu(f)$, where $\nu$ is the unique $\mu$-invariant probability on $F$ (see Lemma 1.2). In this case, there exists a unique minimal absorbing subset (the support of $\nu$ ) without cyclic classes.

In our general case, we have, more precisely, the following results.

2.4. THEOREM ( $\mu$-invariant measures, $\mu$-invariant functions). - Let $G$ be an algebraic Zariski connected real semi-simple group and $\mu$ be a probability measure on $G$. We assume that the Zariski closure of the closed sub-semigroup $T_{\mu}$ generated by the support of $\mu$ is equal to $G$. We denote by $\mathcal{E}$ the set of extremal $\mu$-invariant measures on $X^{\rho}=F \times{ }_{\rho} C$ and we assume $\pi^{\prime}(e) \in L_{T}^{\prime}$. Then, with the above notations:

(i) $\mathcal{E}$ consists of the measures $\sigma_{i}=\hat{\nu} \otimes\left(\lambda * \delta_{c_{i}}\right), 1 \leqslant i \leqslant s$.

(ii) The support $\operatorname{Supp}\left(\sigma_{i}\right)$ of $\sigma_{i}$ is the T-invariant minimal subset $\overline{X_{i}^{\rho}}$.

(iii) For each $\sigma \in \mathcal{E}$ the indicator function $1_{\operatorname{Supp}(\sigma)}$, defined on $\bigcup_{\rho \in \mathcal{E}} \operatorname{Supp}(\rho)$, extends uniquely to $F \times{ }_{\rho} C$ as a continuous $P_{\mu}$-invariant function $h_{\sigma}$. The functions $\left\{h_{\sigma}: \sigma \in \mathcal{E}\right\}$ form a basis of the vector space of $P_{\mu}$-invariant continuous functions on $F \times{ }_{\rho} C$.

2.5. COROLLARY. - The minimal closed subsets of the action of $T$ on $X^{\rho}$ are the sets $\overline{X_{i}^{\rho}}$, $1 \leqslant i \leqslant s$. They are open and closed in $L_{T} \times{ }_{\rho} C$.

2.6. THEOREM (Peripheral spectrum of $P_{\mu}$ ). - Under the hypothesis of Theorem 2.4, we have:

(i) The peripheral spectrum of the operator $P_{\mu}$ is either $\{1\}$ or $\{-1,1\}$.

(ii) If the peripheral spectrum is $\{1\}$, for any $f \in C\left(F \times{ }_{\rho} C\right)$, the sequence of continuous functions $\left(P_{\mu}^{n} f\right)_{n \geqslant 0}$ converges uniformly to $\sum_{\sigma \in \mathcal{E}} \sigma(f) h_{\sigma}$.

(iii) When the peripheral spectrum is $\{-1,1\}$, each T-minimal invariant subset $\operatorname{Supp}(\sigma)$ splits into two "cyclic classes", $C_{\sigma, 1}$ and $C_{\sigma, 2}$, such that $\forall i \in\{0,1\}, \forall x \in C_{\sigma, i}$, $P_{\mu}\left(x, C_{\sigma, i+1 \bmod 2}\right)=1$. For each $\sigma \in \mathcal{E}$ the function $1_{C_{\sigma, 0}}-1_{C_{\sigma, 1}}$, defined on $\bigcup_{\rho \in \mathcal{E}} S_{\rho}$,

$4^{e}$ SÉRIE - TOME $40-2007-\mathrm{N}^{\circ} 2$ 
extends uniquely to $F \times{ }_{\rho} C$ as a continuous function $\tilde{h}_{\sigma}$ such that $P_{\mu} \tilde{h}_{\sigma}=-\tilde{h}_{\sigma}$. The measure $\tilde{\sigma}=\tilde{h}_{\sigma} \sigma$ satisfies $\mu \star \tilde{\sigma}=-\tilde{\sigma}$. For any $f \in C\left(F \times_{\rho} C\right)$, the sequence of continuous functions $\left(P_{\mu}^{2 n} f\right)_{n \geqslant 0}$ converges uniformly to $\sum_{\sigma \in \mathcal{E}}\left(\sigma\left(f h_{\sigma}\right) h_{\sigma}+\sigma\left(f \tilde{h}_{\sigma}\right) \tilde{h}_{\sigma}\right)$.

\section{Proofs of the results}

\section{- Asymptotic behavior of the random walks on a semi-simple Lie group}

3.0. Let $\mu$ be a probability measure on the Borel $\sigma$-algebra $\mathcal{B}(G)$ of $G$ and call $T_{\mu}$ the closed sub-semigroup of $G$ generated by the support $\operatorname{Supp}(\mu)$ of $\mu$ (i.e. $T_{\mu}=\overline{\bigcup_{k \in \mathbb{N}} \operatorname{Supp}\left(\mu^{k}\right)}$ ). We take again the notations of Definition 1.4.

If $G$ acts continuously on a topological space $E$, the convolution $\rho * \nu$ of two probability measures $\rho$ and $\nu$, respectively on $G$ and $E$, is defined by

$$
\int_{E} f(x) \rho * \nu(d x)=\int_{G} \int_{E} f(g \cdot x) \rho(d g) \nu(d x) .
$$

A measure $\nu$ on $E$ is said to be $\rho$-invariant if $\rho * \nu=\nu$. When $E$ is compact, such a measure always exists. For any $u \in E$, we call $\delta_{u}$ the Dirac measure at $u$.

Let $g \in G$ and let $\nu$ be a measure on $E$; we denote by $g \cdot \nu$ the convolution $\delta_{g} * \nu$. When $E=G$ this convolution is simply denoted by $g \nu$.

We recall some results of [17].

3.1. TheOREM. - Suppose that $\mu$ satisfies the hypothesis $(\mathrm{H})$. Then there exists a unique $\mu$-invariant probability measure $\nu$ on $F$ which satisfies for any $g \in G, g \cdot \nu\left(\pi\left(N^{-}\right)\right)=1$.

More precisely, there exists a $\theta$-invariant Borel subset $\Omega_{0}$ of $\Omega$ such that $\mathbb{P}\left[\Omega_{0}\right]=1$ and, for any $\omega \in \Omega_{0}$, the sequence of probability measures $\left(R_{n}(\omega) \cdot \nu\right)_{n \geqslant 1}$ converges towards a Dirac measure $\delta_{Z(\omega)}$ which satisfies $Y_{1}(\omega) \cdot Z(\theta(\omega))=Z(\omega)$. The probability $\nu$ is the law of the random variable $Z$.

From this result we obtain the following theorems (see [17]).

3.2. THEOREM (Behavior in polar decomposition). - Suppose that $\mu$ satisfies the hypothesis $(\mathbf{H})$. For any $\omega \in \Omega$, we write $R_{n}(\omega)=\eta_{n}(\omega) a_{n}(\omega) \kappa_{n}(\omega)$ with $\eta_{n}(\omega), \kappa_{n}(\omega) \in K$ and $a_{n}(\omega) \in A^{+}$. Then there exists a $\theta$-invariant Borel subset $\Omega_{0}$ of $\Omega$ such that $\mathbb{P}\left[\Omega_{0}\right]=1$ and for any $\omega \in \Omega_{0}$ :

(i) $\forall \alpha \in \Delta_{+}, \lim _{n \rightarrow+\infty} \varphi_{\alpha}\left(a_{n}(\omega)\right)=+\infty$,

(ii) $\lim _{n \rightarrow+\infty} \pi\left(\eta_{n}(\omega)\right)=Z(\omega)$.

Using the transformation $g \rightarrow g^{-1}$ we obtain the following result.

3.3. COROLlARY. - For any $\omega \in \Omega$, we write $L_{n}(\omega)=\eta_{n}^{\prime}(\omega) a_{n}^{\prime}(\omega) \kappa_{n}^{\prime}(\omega)$ with $\eta_{n}^{\prime}(\omega)$, $\kappa_{n}^{\prime}(\omega) \in K$ and $a_{n}^{\prime}(\omega) \in A^{-}$. Then there exist a $F^{\prime}$-valued random variable $Z^{\prime}$ and a $\theta$-invariant Borel subset $\Omega_{0}^{\prime}$ of $\Omega$ such that $\mathbb{P}\left[\Omega_{0}^{\prime}\right]=1$ and for any $\omega \in \Omega_{0}^{\prime}$ :

(i) $\forall \alpha \in \Delta_{-}, \lim _{n \rightarrow+\infty} \varphi_{\alpha}\left(a_{n}^{\prime}(\omega)\right)=0$,

(ii) $\lim _{n \rightarrow+\infty} \pi^{\prime}\left(\kappa_{n}^{\prime}(\omega)\right)=Z^{\prime}(\omega)$.

With the notations of Theorem 3.2, the sequence of random variables $\pi^{\prime}\left(\kappa_{n}\right)$ converges in law to $Z^{\prime}$, the law of $Z^{\prime}$ being the unique $\mu$-invariant probability measure $\nu^{\prime}$ on $F^{\prime}$. As for $\nu$ we have $\forall g \in G, \nu^{\prime} \cdot g\left(\pi^{\prime}\left(N^{+}\right)\right)=1$. 
3.4. TheOREM (Behavior in Iwasawa decomposition). - Suppose that $\mu$ satisfies the hypothesis $(\mathrm{H})$. Then, for any $g \in G$ :

(i) the sequences of $N^{-}$-valued random variables $\left(\mathbf{u}\left(g R_{n}\right)\right)_{n \geqslant 1}$ converge, $\mathbb{P}$-a.e., to a $N^{-}$-valued random variable $\hat{Z}(g, \cdot)$ such that $\pi(\hat{Z}(g, \cdot))=g \cdot Z$; as a consequence $\hat{Z}(g, \cdot)=\zeta(g \hat{Z}(e, \cdot)), \mathbb{P}$-a.e.;

(ii) $\forall \alpha \in \Delta_{+}, \lim _{n \rightarrow+\infty} \varphi_{\alpha}\left(\mathbf{b}\left(g R_{n}\right)\right)=+\infty, \mathbb{P}$ a.e.;

(iii) the component $\pi^{\prime}\left(\mathbf{k}\left(g R_{n}\right)\right)$ converges in law to $Z^{\prime}$.

In the same way, we have:

(i) the sequences of $N^{+}$-valued random variables $\left(\mathbf{u}^{\prime}\left(L_{n} g\right)\right)_{n \geqslant 1}$ converge, $\mathbb{P}$-a.e., to a $N^{+}$. valued random variable $\hat{Z}^{\prime}(g, \cdot)$ such that $\pi^{\prime}\left(\hat{Z}^{\prime}(g, \cdot)\right)=Z^{\prime} \cdot g$;

(ii) $\forall \alpha \in \Delta_{+}, \lim _{n \rightarrow+\infty} \varphi_{\alpha}\left(\mathbf{b}^{\prime}\left(L_{n} g\right)\right)=+\infty, \mathbb{P}$ a.e.;

(iii) the component $\pi^{\prime}\left(\mathbf{k}^{\prime}\left(L_{n} g\right)\right)$ converges in law to $Z$.

In the sequel, we note $\hat{Z}(\cdot)$ and $\hat{Z}^{\prime}(\cdot)$ instead of $\hat{Z}(e, \cdot)$ and $\hat{Z}^{\prime}(e, \cdot)$

\section{- Proof of Theorem 2.3}

We denote by $X^{\rho}$ the skew product $X^{\rho}=F \times{ }_{\rho} C$. We endow $X^{\rho}$ with a distance $\delta$ which is $K$-left-invariant and $C$-right-invariant, i.e.

$$
\forall(x, c),(y, d) \in X^{\rho}, \forall(k, f) \in K \times C, \quad \delta(k \cdot(x, c f), k \cdot(y, d f))=\delta((x, c),(y, d)) .
$$

We call $\delta_{C}$ the induced distance on $C$ :

$$
\delta_{C}\left(c, c^{\prime}\right)=\delta\left((\pi(e), c),\left(\pi(e), c^{\prime}\right)\right) .
$$

For $(x, c) \in X^{\rho}$ and $r>0$ we denote by $B((x, c), r)$ the closed ball centered in $(x, c)$ with radius $r$.

The theorem is an immediate consequence of the following result.

3.5. Proposition. - We suppose that $\mu$ satisfies the hypothesis $(\mathrm{H})$. Then:

(i) for any sequences of elements of $K \times C,\left(k_{n}, c_{n}\right)_{n \geqslant 1}$ and $\left(k_{n}^{\prime}, c_{n}^{\prime}\right)_{n \geqslant 1}$, converging respectively to $(k, c)$ and $\left(k^{\prime}, c^{\prime}\right)$,

$$
\begin{aligned}
& \lim _{n \rightarrow+\infty} \delta\left(L_{n} \cdot \overline{\left(k_{n}, c_{n}\right)}, L_{n} \cdot \overline{\left(k_{n}^{\prime}, c_{n}^{\prime}\right)}\right) \\
& \quad=\delta_{C}\left(\rho \circ \gamma\left(\hat{Z}^{\prime} k, \pi(e)\right) c, \rho \circ \gamma\left(\hat{Z}^{\prime} k^{\prime}, \pi(e)\right) c^{\prime}\right), \quad \mathbb{P} \text {-a.e. }
\end{aligned}
$$

(ii) for any $(x, c) \in X^{\rho}$,

$$
\lim _{r \downarrow 0} \limsup _{n \rightarrow+\infty} \sup _{(y, d) \in B((x, c), r)} \mathbb{E}\left[\delta\left(L_{n} \cdot(x, c), L_{n} \cdot(y, d)\right)\right]=0, \quad \mathbb{P} \text {-a.e. }
$$

Consequently, for any $f \in C\left(X^{\rho}\right)$, the family of continuous functions $\left\{P_{\mu}^{n} f: n \geqslant 1\right\}$ is equicontinuous.

Proof. - (i) We write $L_{n}=\mathbf{k}^{\prime}\left(L_{n}\right) \mathbf{b}^{\prime}\left(L_{n}\right) \mathbf{u}^{\prime}\left(L_{n}\right)$ in the Iwasawa decomposition $G=K A N^{+}$ of $G$. From Theorem 3.4, we know that there exists a Borel subset $\Omega_{k}$ of $\Omega$ with $\mathbb{P}\left[\Omega_{k}\right]=1$ such that, for any $\omega \in \Omega_{k}, \mathbf{u}^{\prime}\left(L_{n}(\omega)\right) k_{n}$ converges towards $\hat{Z}^{\prime}(\omega) k \in B$. We can write $\hat{Z}^{\prime}(\omega) k=$ $u_{k}(\omega) a_{k}(\omega) m_{k}(\omega) z_{k}(\omega)$ with $u_{k}(\omega) \in N^{-}, m_{k}(\omega) \in M, a_{k}(\omega) \in A$ and $z_{k}(\omega) \in N^{+}$. Furthermore, for $n$ large enough, $\mathbf{u}^{\prime}\left(L_{n}(\omega)\right) k_{n}$ belongs to the open set $B$ and we can write 
$\mathbf{u}^{\prime}\left(L_{n}(\omega)\right) k_{n}=u_{n, k_{n}}(\omega) m_{n, k_{n}}(\omega) a_{n, k_{n}}(\omega) z_{n, k_{n}}(\omega)$ with $u_{n, k_{n}}(\omega) \in N^{-}, m_{n, k_{n}}(\omega) \in M$, $a_{n, k_{n}}(\omega) \in A$ and $z_{n, k_{n}}(\omega) \in N^{+}$, converging respectively to $u_{k}(\omega), a_{k}(\omega), m_{k}(\omega)$ and $z_{k}(\omega)$.

Hence, we have:

$$
\begin{aligned}
& \left(\mathbf{k}^{\prime}\left(L_{n}\right)\right)^{-1} L_{n}(\omega) \cdot\left(\overline{k_{n}, c_{n}}\right)=\left(\overline{\mathbf{b}^{\prime}\left(L_{n}(\omega)\right) \mathbf{u}^{\prime}\left(L_{n}(\omega)\right) k_{n}, c_{n}}\right) \\
& \quad=\left(\overline{\mathbf{b}^{\prime}\left(L_{n}(\omega)\right) u_{n, x_{n}} \mathbf{b}^{\prime-1}\left(L_{n}(\omega)\right), \rho\left(\mathbf{b}^{\prime}\left(L_{n}(\omega)\right) m_{n, k_{n}}(\omega) a_{n, k_{n}}(\omega)\right) c}\right) .
\end{aligned}
$$

As the sequence $y_{n}(\omega)=\mathbf{b}^{\prime}\left(L_{n}(\omega)\right) u_{n, x_{n}}(\omega) \mathbf{b}^{\prime-1}\left(L_{n}(\omega)\right)$ converges towards $e$, for $\omega \in \Omega_{k} \cap$ $\Omega_{y}$ and for $n$ large enough, $\delta\left(L_{n}(\omega) \cdot\left(\overline{k_{n}, c_{n}}\right), L_{n}(\omega) \cdot\left(\overline{k_{n}^{\prime}, c_{n}^{\prime}}\right)\right)$ converges towards

$$
\begin{aligned}
& \delta\left(\left(\pi(e), \rho\left(m_{k}(\omega) a_{k}(\omega)\right) c\right),\left(\pi(e), \rho\left(m_{k^{\prime}}(\omega) a_{k^{\prime}}(\omega)\right) c^{\prime}\right)\right) \\
& \quad=\delta\left(\left(\pi(e), \rho\left(\gamma\left(\hat{Z}^{\prime}(\omega) k, \pi(e)\right)\right) c\right),\left(\pi(e), \rho\left(\gamma\left(\hat{Z}^{\prime}(\omega) k^{\prime}, \pi(e)\right)\right) c^{\prime}\right)\right) .
\end{aligned}
$$

(ii) For any $(k, c) \in K \times C, r>0$ and $n \geqslant 1$, we have:

$$
\sup _{\left(\overline{k^{\prime}, c^{\prime}}\right) \in B((\overline{k, c}), r)} \mathbb{E}\left[\delta\left(L_{n} \cdot(\overline{k, c}), L_{n} \cdot\left(\overline{k^{\prime}, c^{\prime}}\right)\right)\right]=\mathbb{E}\left[\delta\left(L_{n} \cdot(\overline{k, c}), L_{n} \cdot\left(\overline{k_{n}, c_{n}}\right)\right)\right]
$$

for some $\left(k_{n}, c_{n}\right) \in K \times C$ such that $\delta\left((\overline{k, c}),\left(\overline{k_{n}, c_{n}}\right)\right) \leqslant r$. According to the first assertion, any closure values of the sequence on the right hand of the equality is given by $\mathbb{E}\left[\delta_{C}\left(\rho\left(\gamma\left(\hat{Z}^{\prime} k, \pi(e)\right)\right) c, \rho\left(\gamma\left(\hat{Z}^{\prime} k^{\prime \prime}, \pi(e)\right) c^{\prime \prime}\right)\right]\right.$ where $\left(k^{\prime \prime}, c^{\prime \prime}\right)$ is a closure value of the sequence $\left(k_{n}, c_{n}\right)$. Consequently,

$$
\begin{aligned}
& \limsup _{n \rightarrow+\infty} \sup _{\left(\overline{k^{\prime}, c^{\prime}}\right) \in B(\overline{(\overline{k, c}), r})} \mathbb{E}\left[\delta\left(L_{n} \cdot(\overline{k, c}), L_{n} \cdot\left(\overline{k^{\prime}, c^{\prime}}\right)\right)\right] \\
& \leqslant \sup _{\left(\overline{k^{\prime}, c^{\prime}}\right) \in B(\overline{k, c}, r)} \mathbb{E}\left[\delta_{C}\left(\rho\left(\gamma\left(\hat{Z}^{\prime} k, \pi(e)\right)\right) c, \rho\left(\gamma\left(\hat{Z}^{\prime} k^{\prime}, \pi(e)\right)\right) c^{\prime}\right)\right] .
\end{aligned}
$$

Now we prove that

$$
\lim _{r \rightarrow 0} \sup _{\left.\left(\overline{k^{\prime}, c^{\prime}}\right) \in B(\overline{(k, c}), r\right)} \mathbb{E}\left[\delta_{C}\left(\rho\left(\gamma\left(\hat{Z}^{\prime} k, \pi(e)\right)\right) c, \rho\left(\gamma\left(\hat{Z}^{\prime} k^{\prime}, \pi(e)\right)\right) c^{\prime}\right)\right]=0 .
$$

Let $\left(r_{n}\right)_{n \geqslant 1}$ be a sequence of real numbers decreasing to 0 . For any $n \geqslant 1$, we set

$$
\Omega_{n}=\left\{\omega \in \Omega_{k}: \forall\left(\overline{k^{\prime}, c^{\prime}}\right) \in B\left((\overline{k, c}), r_{n}\right), \hat{Z}(\omega) k^{\prime} \in B\right\} .
$$

When $n \uparrow+\infty, \Omega_{n}$ increases to $\Omega_{k}$ and for any integers $p, n$ verifying $n \geqslant p$, we have:

$$
\begin{aligned}
& \quad \sup _{\left(\overline{k^{\prime}, c^{\prime}}\right) \in B\left(\overline{\left.(\overline{k, c}), r_{n}\right)}\right.} \mathbb{E}\left[\delta_{C}\left(\rho\left(\gamma\left(\hat{Z}^{\prime} k, \pi(e)\right)\right) c, \rho\left(\gamma\left(\hat{Z}^{\prime} k^{\prime}, \pi(e)\right)\right) c^{\prime}\right)\right] \\
& \quad \leqslant \sup _{\left(\overline{k^{\prime}, c^{\prime}}\right) \in B\left(\overline{(k, c)}, r_{n}\right)} \mathbb{E}\left[\delta_{C}\left(\rho\left(\gamma\left(\hat{Z}^{\prime} k, \pi(e)\right)\right) c, \rho\left(\gamma\left(\hat{Z}^{\prime} k^{\prime}, \pi(e)\right)\right) c^{\prime}\right) 1_{\Omega_{p}}\right]+\|\delta\|_{\infty} \mathbb{P}\left[\Omega_{p}^{c}\right] .
\end{aligned}
$$

The continuity of $\gamma$ on $B$ implies that, for any $p \geqslant 1$,

$$
\limsup _{n \rightarrow+\infty} \sup _{\left.\left(\overline{k^{\prime}, c^{\prime}}\right) \in B(\overline{(k, c}), r_{n}\right)} \mathbb{E}\left[\delta_{C}\left(\rho\left(\gamma\left(\hat{Z}^{\prime} k, \pi(e)\right)\right) c, \rho\left(\gamma\left(\hat{Z}^{\prime} k^{\prime}, \pi(e)\right)\right) c^{\prime}\right)\right] \leqslant\|\delta\|_{\infty} \mathbb{P}\left[\Omega_{p}^{c}\right]
$$


and therefore

$$
\limsup _{n \rightarrow+\infty} \sup _{\left(\overline{k^{\prime}, c^{\prime}}\right) \in B\left(\overline{(k, c), r_{n}}\right)} \mathbb{E}\left[\delta_{C}\left(\rho\left(\gamma\left(\hat{Z}^{\prime} k, \pi(e)\right)\right) c, \rho\left(\gamma\left(\hat{Z}^{\prime} k^{\prime}, \pi(e)\right)\right) c^{\prime}\right)\right]=0 .
$$

In order to show the last assertion, we can assume, by a density argument, that $f$ satisfies

$$
\sup _{(x, c),(y, d) \in X^{\rho}} \frac{|f(x, c)-f(y, d)|}{\delta((x, c),(y, d))}=[f]<+\infty .
$$

We have

$$
\left|P_{\mu}^{n} f(x, c)-P_{\mu}^{n} f(y, d)\right| \leqslant[f] \mathbb{E}\left[\delta\left(L_{n} \cdot(x, c), L_{n} \cdot(y, d)\right)\right]
$$

and the result is clear according to the assertion (ii).

In the proof above, if we replace $X^{\rho}$ by $F=G / M A N^{+}$we obtain, on the one hand,

$$
\lim _{n \rightarrow+\infty} \delta\left(L_{n} \cdot \pi\left(k_{n}\right), L_{n} \cdot \pi\left(k_{n}^{\prime}\right)\right)=0 \quad \mathbb{P} \text {-a.e., }
$$

which proves the proximality of the trajectories of the left random walk on $F$ and, on the other hand, for any $f \in C(F)$, the relative compacity of the family $\left\{P_{\mu}^{n} f: n \in \mathbb{N}\right\}$ in $\left(C(F),\|\|_{\infty}\right)$. Then from the inequalities

$$
\forall y \in \operatorname{Supp}(\nu), \quad \delta\left(L_{n} \cdot x, \operatorname{Supp}(\nu)\right) \leqslant \delta\left(L_{n} \cdot x, L_{n} \cdot y\right)
$$

and

$$
\left|\mathbb{E}\left[f\left(L_{n} \cdot x\right)-\nu(f)\right]\right| \leqslant \int_{F} \mathbb{E}\left[\left|f\left(L_{n} \cdot x\right)-f\left(L_{n} \cdot y\right)\right|\right] \nu(d y),
$$

we obtain the following well-known result:

3.6. Corollary. - For any $x \in F, \lim _{n \rightarrow+\infty} \delta\left(L_{n} \cdot x, \operatorname{Supp}(\nu)\right)=0 \mathbb{P}$-a.e.

For any $f \in C(F)$, the sequence of functions $\left(P_{\mu}^{n} f\right)_{n \geqslant 0}$ converges uniformly on $F$ to $\nu(f)$.

\section{- Proof of Proposition 1.5}

Let $Y$ be a closed $T$-invariant subset of $F$ and $\mu$ a probability measure on $G$ such that $T_{\mu}=T$. From the Markov-Kakutani theorem, there exists a $\mu$-stationary probability measure $\nu$ on $F$ supported by $Y$. From Lemma 1.2, $\nu$ is unique therefore it satisfies $\nu\left(L_{T}\right)=1$ and $\operatorname{Supp}(\nu) \subset L_{T}$. On the other hand, since $\nu$ is irreducible,

$$
\forall g \in T^{\mathrm{prox}}, \quad \lim _{n} g^{n} \cdot \nu=\delta_{g^{-}}
$$

and therefore $L_{T} \subset \operatorname{Supp}(\nu) \subset Y$. Hence $L_{T}=\operatorname{Supp}(\nu)$ and $\forall x \in L_{T}, \overline{T \cdot x}=L_{T}$. Therefore, $L_{T}$ is the unique $T$-minimal subset.

We know from Corollary 3.6 that, for any $x \in F, \lim _{n} \delta\left(L_{n} \cdot x, \operatorname{Supp}(\nu)\right)=0, \mathbb{P}$-a.e. It follows that any $\mu$-recurrent point $x$ of $F$ belongs to $\operatorname{Supp}(\nu)$.

We consider the space $F \times \Omega$ provided with the measure $\nu \otimes \mathbb{P}$ and the transformation $\tilde{\theta}$ of $F \times \Omega$ defined by $\tilde{\theta}(x, \omega)=\left(Y_{1}(\omega) \cdot x, \theta(\omega)\right)$. The probability $\nu \otimes \mathbb{P}$ is $\tilde{\theta}$-invariant and 
ergodic (see [29], Cor. (1.8)). The ergodic theorem applied to this system and the function $(x, \omega) \rightarrow 1_{B(x, r)}\left(L_{n}(\omega) \cdot x\right)$ give us:

$$
\lim _{n \rightarrow+\infty} \frac{1}{n} \sum_{k=1}^{n} 1_{B(x, r)}\left(L_{n}(\omega) \cdot x\right)=\nu(B(x, r))
$$

for $\nu \otimes \mathbb{P}$-almost every $(x, \omega) \in F \times \Omega$. If $x$ belongs to the support $\operatorname{Supp}(\nu)$ of $\nu$, we have $\nu(B(x, r))>0$, for any $r>0$. Therefore, for $\nu \otimes \mathbb{P}$-almost every $(x, \omega) \in \operatorname{Supp}(\nu) \times \Omega$, we conclude:

$$
\forall p \geqslant 1, \quad \sum_{n \in \mathbb{N}} 1_{B(x, 1 / p)}\left(L_{n}(\omega) \cdot x\right)=+\infty
$$

which proves that $\nu$-almost every $x \in \operatorname{Supp}(\nu)$ is $\mu$-recurrent. The proximality of the trajectories of the left random walk on $F$ shown above, implies that any element of $\operatorname{Supp}(\nu)$ is $\mu$-recurrent.

Since $\nu$ is irreducible, we have $\nu(\pi(P))=0$. Since $\operatorname{Supp}(\nu)=L_{T}$, we have $\pi(e) \in \operatorname{Supp}(\nu)$, hence there exists a sequence $\left(X_{n}\right)$ as in the proposition. We observe that a somewhat more direct proof can be given using the Zariski density of $T$, instead of hypothesis $(\mathrm{H})$. The set $Y$ of points $y=\pi(g) \in L_{T}$, such that there exists a neighborhood $V^{y}$ of $y$ contained in $\pi(g P)$, is open in $L_{T}$ and $T$-invariant. From the minimality of $L_{T}$ we deduce $Y=\emptyset$ or $Y=L_{T}$. The first condition gives the required assertion. If the second condition is satisfied, there exists a finite covering of $L_{T}$ by open neighborhoods $V^{y_{i}}, y_{i}=\pi\left(g_{i}\right) \in L_{T}, 1 \leqslant i \leqslant p$. Then $L_{T} \subset \bigcup_{i=1}^{p} \pi\left(g_{i} P\right)$ and this contradicts the Zariski density of $L_{T}$ in $F$.

\section{- Proof of Proposition 1.8}

(1) Let $x \in L_{T} \cap g^{-1} \cdot \pi\left(N^{-}\right)$and $s \in T$ such that $y=s \cdot x \in L_{T} \cap g^{-1} \cdot \pi\left(N^{-}\right)$. For any $t \in T$ such that $t \cdot y \in L_{T} \cap g^{-1} \cdot \pi\left(N^{-}\right)$, we have $\gamma_{g}(t s, x)=\gamma_{g}(t, y) \gamma_{g}(s, x)$. Hence $\gamma_{g}(t, y)$ belongs to $\Gamma_{g, x}(T)$ and $\Gamma_{g, y}(T) \subset \Gamma_{g, x}(T)$.

Now let $z \in L_{T} \cap g^{-1} \cdot \pi\left(N^{-}\right)$, since $T \cdot x$ is dense in $L_{T}$ and $g^{-1} \cdot \pi\left(N^{-}\right)$is open in $L_{T}$, there exists a sequence $\left(s_{n}\right)_{n \geqslant 0}$ of elements of $T$ such that $\forall n \in \mathbb{N}, s_{n} \cdot x \in L_{T} \cap g^{-1} \cdot \pi\left(N^{-}\right)$and $s_{n} \cdot x \rightarrow z$. For any $n \in \mathbb{N}, \Gamma_{g, s_{n} \cdot x}(T) \subset \Gamma_{g, x}(T)$. Let $t \in T$ such that $t . z \in L_{T} \cap g^{-1} \cdot \pi\left(N^{-}\right)$, then for $n$ large enough, $t s_{n} \cdot x \in L_{T} \cap g^{-1} \cdot \pi\left(N^{-}\right)$and $\gamma_{g}(t, z)=\lim _{n} \gamma_{g}\left(t, s_{n} \cdot x\right) \in \Gamma_{g, x}(T)$; consequently $\Gamma_{g, z}(T) \subset \Gamma_{g, x}(T)$. Hence $\Gamma_{g, x}(T)$ is independent of $x \in L_{T} \cap g^{-1} \cdot \pi\left(N^{-}\right)$and is equal to $\Gamma_{g}(T)$.

(2) We can repeat the same argument as above for $\operatorname{pr}\left(\Gamma_{g}(T)\right)$ when $\pi^{\prime}(g)$ describes $L_{T}^{\prime}$. Then we get that $\operatorname{pr}\left(\Gamma_{g}(T)\right)$ is independent of $g \in \pi^{\prime-1}\left(L_{T}^{\prime}\right)$.

We call $S$ the sub-semigroup $g T g^{-1}$. It is clear that $L_{S}=g \cdot L_{T}$. Hence $\Gamma_{g}(T)=\Gamma_{e}(S)$.

(3) Let $t \in T^{\text {prox }}$; we write $t=s^{-1}$ mas with $m a \in M A^{+}$and $s \in G$. Let $g \in G$ satisfying $g \cdot t^{-} \in \pi\left(N^{-}\right),\left(t^{-}=\pi\left(s^{-1}\right) \in L_{T}\right)$. Then $\gamma_{g}\left(t, t^{-}\right)=a m$ modulo $[M, M]$. This implies: $\operatorname{pr}\left(\gamma_{g}\left(t, t^{-}\right)\right)=\lambda\left(g t g^{-1}\right)=\lambda(t)$ and $\lambda(t) \in \operatorname{pr}\left(\Gamma_{g}(T)\right)$ for any $g \in G$ such that $g \cdot t^{-} \in \pi\left(N^{-}\right)$.

Let $t \in T^{\text {prox }}$ and $g \in G$. For any $\varepsilon>0$ we can choose $\left(g_{\varepsilon}, u_{\varepsilon}\right) \in G \times L_{T}$ such that $g_{\varepsilon}$. $t^{-} \in \pi\left(N^{-}\right), g \cdot u_{\varepsilon} \in \pi\left(N^{-}\right)$and $d\left(\operatorname{pr}\left(\gamma_{g}\left(t, u_{\varepsilon}\right)\right), \operatorname{pr}\left(\gamma_{g_{\varepsilon}}\left(t, t^{-}\right)\right)\right)=d\left(\operatorname{pr}\left(\gamma_{g}\left(t, u_{\varepsilon}\right)\right), \lambda(t)\right)<\varepsilon$. Then, for any $\varepsilon>0$, we have $d\left(\lambda(t), \operatorname{pr}\left(\Gamma_{g}(T)\right)\right)<\varepsilon$. As $\operatorname{pr}\left(\Gamma_{g}(T)\right)$ is closed we deduce that $\lambda(t) \in \operatorname{pr}\left(\Gamma_{g}(T)\right)$.

Hence, for any $g \in G$, $\langle\operatorname{Spec} T\rangle \subset \operatorname{pr}\left(\Gamma_{g}(T)\right)$.

(4) Let $t \in T^{\text {prox }}$, we write $t=g^{-1}$ mag with $m a \in M A^{+}$and $g \in G$. We set $x=t^{-}=$ $\pi\left(g^{-1}\right)$. Let $s \in T$ such that $g s \cdot x \in \pi\left(N^{-}\right)$. We know that, for $n$ large enough, $t^{n} s$ belongs to $T^{\text {prox }}$ and $x_{n}=\left(t^{n} s\right)^{-}$converges to $x$. As $g \cdot x=\pi(e)$, for $n$ large enough, we have 
$g \cdot x_{n} \in \pi\left(N^{-}\right), g s \cdot x_{n} \in \pi\left(N^{-}\right)$and

$$
\lambda\left(t^{n} s\right)=\operatorname{pr}\left(\gamma_{g}\left(t^{n} s, x_{n}\right)\right)=\operatorname{pr}\left(\gamma_{g}\left(t^{n}, s \cdot x_{n}\right)\right) \operatorname{pr}\left(\gamma_{g}\left(s, x_{n}\right)\right)=\lambda\left(t^{n}\right) \operatorname{pr}\left(\gamma_{g}\left(s, x_{n}\right)\right) .
$$

We deduce that

$$
\operatorname{pr}\left(\gamma_{g}(s, x)\right)=\lim _{n \rightarrow+\infty} \operatorname{pr}\left(\gamma_{g}\left(s, x_{n}\right)\right)=\lim _{n \rightarrow+\infty} \lambda\left(t^{n} s\right)\left(\lambda\left(t^{n}\right)\right)^{-1} ;
$$

which proves that $\operatorname{pr}\left(\Gamma_{g, x}(T)\right) \subset\langle\operatorname{Spec} T\rangle$. Hence the result.

For the proof of Theorem 1.9, we need some intermediate results.

3.7. LemmA. - For $g \in G$, the subgroup $\Gamma_{g}(T)$ is Zariski dense in $M A$ and contains the commutator subgroup $[M, M]$ of $M$. Furthermore $[M, M]$ is connected.

Proof. - We set $U=\left\{(t, x) \in T \times F: x\right.$ and $t \cdot x$ belong to $\left.g^{-1} \cdot \pi\left(N^{-}\right)\right\}$. The function $\gamma_{g}$ is rational on $U$ and the subgroup $M A$ is algebraic.

Since $\mathcal{Z}(T)=G$ and the subgroup $\Gamma_{g}(T)$ is generated by the elements $\gamma_{g}(t, x),(t, x) \in$ $\left(T \times L_{T}\right) \cap U$, we have:

$$
\mathcal{Z}\left(\Gamma_{g}(T)\right)=\mathcal{Z}\left(\Gamma_{g}(G)\right)=M A
$$

and

$$
\mathcal{Z}\left(\left[\Gamma_{g}(T), \Gamma_{g}(T)\right]\right)=\mathcal{Z}([M A, M A])=\mathcal{Z}([M, M]) .
$$

We observe that $[M, M]=[M A, M A]$ is the group of real points of the commutator group of $Z_{\mathbf{G}}(A)$, the centralizer of $A$ in $\mathbf{G}$, hence $[M, M]$ is Zariski closed. Also, since the commutator group of $Z_{\mathbf{G}}(A)$ is Zariski connected and $\mathbb{R}$-anisotropic, the compact group $[M, M]$ is connected (see [9], pp. 152-157). Finally, since the commutator group of $\Gamma_{g}(T)$ is Zariski dense in $[M, M]$, this subgroup is also dense in $[M, M]$ in the usual topology and $\Gamma_{g}(T)$ contains $[M, M]$.

Let $\mathfrak{v}$ be a subset of $\mathfrak{n}^{-}$and $U \in \mathfrak{v}$. We denote by $\mathfrak{v}^{U}$ the set of vectors $X \in \mathfrak{n}^{-}$for which there exist a sequence $\left(\beta_{n}\right)_{n \geqslant 0}$ of real numbers and a sequence of vectors $\left(X_{n}\right)_{n \geqslant 0}$ of $\mathfrak{v}$ such that

$$
X_{n} \underset{n \rightarrow+\infty}{\longrightarrow} U \text { and } \beta_{n}\left(X_{n}-U\right) \underset{n \rightarrow+\infty}{\longrightarrow} X .
$$

Then $\mathfrak{v}^{U}$ is a closed $\mathbb{R}^{*}$-homogeneous subset of $\mathfrak{n}^{-}$.

3.8. LEMMA. - Let $T$ be a Zariski dense sub-semigroup of $G$ such that $\pi(e) \in L_{T}$ and $\pi^{\prime}(e) \in L_{T}^{\prime}$. We denote $H=\Gamma_{e}(T)$ and we call $\mathfrak{h}$ the Lie algebra of $H$.

We set $\mathfrak{v}_{T}=\left\{U \in \mathfrak{n}^{-}: \exp U \in L_{T}\right\}$. Then $\operatorname{Ad}\left(M A N^{+}\right)\left(\mathfrak{v}_{T}^{0}\right) \subset \mathfrak{n}^{-} \oplus \mathfrak{h} \oplus \mathfrak{n}^{+}$and consequently

$$
\forall k \in \mathbb{N}, \quad\left(\operatorname{ad}\left(\mathfrak{m} \oplus \mathfrak{a} \oplus \mathfrak{n}^{+}\right)\right)^{k}\left(\mathfrak{v}_{T}^{0}\right) \subset \mathfrak{n}^{-} \oplus \mathfrak{h} \oplus \mathfrak{n}^{+} .
$$

Proof. - Let $X \in \mathfrak{v}_{T}^{0}$. There exist a sequence $\left(X_{n}\right)_{n \geqslant 0}$ of elements of $\mathfrak{v}_{T}$ converging to 0 and a sequence of positive real numbers $\left(\beta_{n}\right)_{n \geqslant 0}$ such that $\beta_{n} X_{n} \underset{n \rightarrow+\infty}{\longrightarrow} X$.

For any $s \in T \cap B$ and any $n$ large enough,

$$
\gamma(s, \pi(e))^{-1} \gamma\left(s, \pi\left(\exp \left(X_{n}\right)\right)\right)=\gamma\left(\eta(s), \pi\left(\exp \left(X_{n}\right)\right)\right) \in H
$$

which implies

$$
\eta(s) \exp \left(X_{n}\right)(\eta(s))^{-1}=\exp \left(\operatorname{Ad} \eta(s)\left(X_{n}\right)\right) \in N^{-} H N^{+} .
$$

$4^{e}$ SÉRIE - TOME $40-2007-\mathrm{N}^{\circ} 2$ 
We introduce on $\mathfrak{g}$ the "product", given by the Campbell-Hausdorff formula,

$$
U \circ V=U+V+\frac{1}{2}[U, V]+\frac{1}{12}[U,[U, V]]-\frac{1}{12}[V,[V, U]]+\cdots .
$$

For any $U$ and $V$ in $\mathfrak{g}$, near to 0 , we have $\exp (U \circ V)=\exp U \exp V$. So, for $n$ large enough, we can write:

$$
\exp \left(\operatorname{Ad} \eta(s)\left(X_{n}\right)\right)=\exp \left(U_{n}\right) \exp \left(V_{n}\right)=\exp \left(U_{n} \circ V_{n}\right)
$$

where $\left(U_{n}\right)_{n \geqslant 0}$ [resp. $\left(V_{n}\right)_{n \geqslant 0}$ ] is a sequence of elements of $\mathfrak{n}^{-}$[resp. $\mathfrak{h} \oplus \mathfrak{n}^{+}$] converging towards 0 . Consequently

$$
\operatorname{Ad} \eta(s)\left(X_{n}\right)=U_{n} \circ V_{n}=U_{n}+V_{n}+R_{n}
$$

where $R_{n} \in \mathfrak{g}$ has a norm $\left\|R_{n}\right\|=\mathrm{o}\left(\left\|U_{n}\right\|+\left\|V_{n}\right\|\right)$. Since $\beta_{n} X_{n}$ converges to $X$, it follows that $\beta_{n}\left(U_{n}+V_{n}\right)$ converges and $\operatorname{Ad} \eta(s)(X) \in \mathfrak{n}^{-} \oplus \mathfrak{h} \oplus \mathfrak{n}^{+}$.

Hence we have proved that:

$$
\forall s \in T \cap B, \forall X \in \mathfrak{v}_{T}^{0}, \quad \operatorname{Ad} \eta(s)(X) \in \mathfrak{n}^{-} \oplus \mathfrak{h} \oplus \mathfrak{n}^{+} .
$$

Since the map $s \in B \rightarrow \operatorname{Ad}(\eta(s))(X) \in \mathfrak{g}$ is rational and $\mathcal{Z}(T)=G$, we get:

$$
\forall X \in \mathfrak{v}_{T}^{0}, \quad \operatorname{Ad} N^{+}(X) \in \mathfrak{n}^{-} \oplus \mathfrak{h} \oplus \mathfrak{n}^{+} .
$$

Since $\operatorname{Ad}(M A)$ preserves $\mathfrak{n}^{-}$and $X \in \mathfrak{n}^{-}$, we have:

$$
\operatorname{Ad}(M A)(X) \subset \mathfrak{n}^{-} \oplus \mathfrak{h} \oplus \mathfrak{n}^{+} .
$$

Furthermore, from Lemma 3.7, we know that $H$ contains $[M, M]$, hence $\mathfrak{h}$ is an ideal of $\mathfrak{m} \oplus \mathfrak{a}$ and $\operatorname{Ad}(M A)(\mathfrak{h})=\mathfrak{h}$. Finally, for any $X \in \mathfrak{v}_{T}^{0}$ :

$$
\operatorname{Ad}\left(M A N^{+}\right)(X) \subset \operatorname{Ad}(M A)\left(\mathfrak{n}^{-} \oplus \mathfrak{h} \oplus \mathfrak{n}^{+}\right) \subset \mathfrak{n}^{-} \oplus \mathfrak{h} \oplus \mathfrak{n}^{+} .
$$

Hence the result.

From the above lemma, the subspace $E=\sum_{k \geqslant 0} \operatorname{ad}^{k}\left(\mathfrak{m} \oplus \mathfrak{a} \oplus \mathfrak{n}^{+}\right)\left(\mathfrak{v}_{T}^{0}\right)$ is contained in $\mathfrak{n}^{-} \oplus \mathfrak{h} \oplus \mathfrak{n}^{+}$and is $\operatorname{ad}\left(\mathfrak{m} \oplus \mathfrak{a} \oplus \mathfrak{n}^{+}\right)$-invariant. In the following lemmas we compare the subalgebras $\mathcal{L}$ generated by $E$ with the standard parabolic subalgebras which contain $\mathcal{L}$. From the properties of $T, L_{T}$ and $\mathfrak{v}_{T}^{0}$, this will allow to show $\mathfrak{h}=\mathfrak{m} \oplus \mathfrak{a}$.

3.9. LEMMA. - Let $\mathfrak{h}$ be a subalgebra of $\mathfrak{m} \oplus \mathfrak{a}$ and $E$ be an $\operatorname{ad}\left(\mathfrak{m} \oplus \mathfrak{a} \oplus \mathfrak{n}^{+}\right)$-invariant subspace of $\mathcal{G}$, contained in $\mathfrak{n}^{-} \oplus \mathfrak{h} \oplus \mathfrak{n}^{+}$. Then the Lie algebra $\mathcal{L}$ generated by $E$ is also $\operatorname{ad}\left(\mathfrak{m} \oplus \mathfrak{a} \oplus \mathfrak{n}^{+}\right)$-invariant and contained in $\mathfrak{n}^{-} \oplus \mathfrak{h} \oplus \mathfrak{n}^{+}$.

Proof. - Consider the subsets $\left(A_{n}\right)_{n \geqslant 1}$ of $\mathcal{L}$ defined by

$$
A_{1}=E \quad \text { and for } n \geqslant 2, A_{n}=\left\{\left[U, A_{n-1}\right]: U \in E\right\} .
$$

The Lie algebra $\mathcal{L}$ is the subspace generated by these subsets. For any $n \geqslant 1$, we denote by $\left\langle A_{n}\right\rangle$ the subspace generated by $A_{n}$. For any $n \geqslant 1$, consider the property:

$$
P(n)=\left\{\forall Y \in \mathfrak{m} \oplus \mathfrak{a} \oplus \mathfrak{n}^{+},\left[Y, A_{n}\right] \subset\left\langle A_{n}\right\rangle\right\} .
$$


Clearly $P(1)$ is true. Using the Jacobi identity

$$
\forall Y \in \mathfrak{m} \oplus \mathfrak{a} \oplus \mathfrak{n}^{+}, \forall U \in E, \forall Z \in A_{n-1}, \quad[Y,[U, Z]]=[[Y, U], Z]+[U,[Y, Z]],
$$

one proves by induction that $P(n)$ is true for any $n \geqslant 1$. Hence the subalgebra $\mathcal{L}$ is ad $(\mathfrak{m} \oplus \mathfrak{a} \oplus$ $\mathfrak{n}^{+}$)-invariant.

For any $n \geqslant 1$, consider now the property:

$$
Q(n)=\left\{A_{n} \subset \mathfrak{n}^{-} \oplus \mathfrak{h} \oplus \mathfrak{n}^{+}\right\} .
$$

From hypothesis $Q(1)$ is true. Assume that $Q(n)$ is true for some integer $n \geqslant 1$. For any $U \in E$ and $Z \in A_{n}$, let $Y_{1}$ and $Y_{2}$ be the elements of $\mathfrak{n}^{+}$such that $U+Y_{1}$ and $Z+Y_{2}$ belong to $\mathfrak{n}^{-} \oplus \mathfrak{h}$. We have $[U, Z]=\left[U+Y_{1}, Z+Y_{2}\right]-\left[Y_{1}, Z\right]+\left[Y_{2}, U\right]-\left[Y_{1}, Y_{2}\right]$ which proves, via properties $Q(n)$ and $P(n)$, that $Q(n+1)$ is true. So $Q(n)$ is true for any $n \geqslant 1$. Hence the result.

3.10. LemmA. - Let $\mathfrak{h}$ be a subalgebra of $\mathfrak{m} \oplus \mathfrak{a}$. We denote by $\mathfrak{S}$ the set of subalgebras $\mathcal{L}$ of $\mathfrak{g}$ satisfying the two following conditions:

$$
\mathcal{L} \subset \mathfrak{n}^{-} \oplus \mathfrak{h} \oplus \mathfrak{n}^{+}, \quad\left[\mathfrak{m} \oplus \mathfrak{a} \oplus \mathfrak{n}^{+}, \mathcal{L}\right] \subset \mathcal{L} .
$$

Then we have:

(i) For any $\mathcal{L} \in \mathfrak{S}, \mathcal{P}=\mathfrak{m} \oplus \mathfrak{a} \oplus \mathfrak{n}^{+}+\mathcal{L}$ is a standard parabolic subalgebra of $\mathfrak{g}$ and $\mathcal{L}+\mathfrak{h}+\mathfrak{n}^{+}$is a subalgebra equal to $\left(\mathcal{P} \cap \mathfrak{n}^{-}\right) \oplus \mathfrak{h} \oplus \mathfrak{n}^{+}$. In particular, if $\mathfrak{h} \neq \mathfrak{m} \oplus \mathfrak{a}$ then $\mathcal{P} \neq \mathfrak{g}$. Moreover, if $\mathfrak{h}$ is an ideal of $\mathfrak{m} \oplus \mathfrak{a}$ and $\mathcal{L}$ belongs to $\mathfrak{S}$, then the subalgebra $\mathcal{L}+\mathfrak{h}+\mathfrak{n}^{+}$ belongs also to $\mathfrak{S}$.

(ii) For any $\left(\mathcal{L}, \mathcal{L}^{\prime}\right) \in \mathfrak{S} \times \mathfrak{S}$, the subalgebra of $\mathfrak{g}$ generated by $\mathcal{L} \cup \mathcal{L}^{\prime}$ belongs to $\mathfrak{S}$. Therefore $\mathfrak{S}$ contains a largest subalgebra. If $\mathfrak{h}$ is an ideal of $\mathfrak{m} \oplus \mathfrak{a}$, this subalgebra is (with obvious notation) equal to $\left(\mathcal{P} \cap \mathfrak{n}^{-}\right) \oplus \mathfrak{h} \oplus \mathfrak{n}^{+}$.

Proof. - (i) From the definition of $\mathcal{P}$ and the decomposition $\mathfrak{g}=\mathfrak{n}^{-} \oplus \mathfrak{m} \oplus \mathfrak{a} \oplus \mathfrak{n}^{+}$we get the equality of the projection on $\mathfrak{n}^{-}$of $\mathcal{L}$ and $\mathcal{P}$. Since $\mathcal{L}$ is $\operatorname{ad}\left(\mathfrak{m} \oplus \mathfrak{a} \oplus \mathfrak{n}^{+}\right)$-invariant, $\mathcal{P}$ is a subalgebra and consequently a standard parabolic subalgebra of $\mathfrak{g}$. It follows that $\mathcal{P}=\left(\mathcal{P} \cap \mathfrak{n}^{-}\right) \oplus \mathfrak{m} \oplus \mathfrak{a} \oplus \mathfrak{n}^{+}$. On the other hand, we have:

$$
\mathcal{L} \subset \mathfrak{n}^{-} \oplus \mathfrak{h} \oplus \mathfrak{n}^{+}, \quad \mathfrak{h} \oplus \mathfrak{n}^{+} \subset \mathfrak{m} \oplus \mathfrak{a} \oplus \mathfrak{n}^{+} .
$$

Hence the projection of $\mathcal{L}$ on $\mathfrak{n}^{-}$modulo $\mathfrak{h} \oplus \mathfrak{n}^{+}$is equal to $\mathcal{P} \cap \mathfrak{n}^{-}$. In other words, $\mathcal{L}+\mathfrak{h}+\mathfrak{n}^{+}=\left(\mathcal{P} \cap \mathfrak{n}^{-}\right) \oplus \mathfrak{h} \oplus \mathfrak{n}^{+}$. Furthermore, since

$$
\left[\mathfrak{h} \oplus \mathfrak{n}^{+}, \mathfrak{h} \oplus \mathfrak{n}^{+}\right] \subset \mathfrak{h} \oplus \mathfrak{n}^{+} \quad \text { and } \quad\left[\mathfrak{h} \oplus \mathfrak{n}^{+}, \mathcal{L}\right] \subset \mathcal{L}
$$

the subspace $\mathcal{L}+\mathfrak{h}+\mathfrak{n}^{+}$is a subalgebra.

If $\mathcal{P}=\mathfrak{g}$ then $\mathcal{P} \cap \mathfrak{n}^{-}=\mathfrak{n}^{-}$. Since $\mathfrak{n}^{-} \oplus \mathfrak{n}^{+}$generates $\mathfrak{g}$ as an algebra, we get $\mathcal{P} \cap \mathfrak{n}^{-} \oplus \mathfrak{h} \oplus$ $\mathfrak{n}^{+}=\mathfrak{g}$ and $\mathfrak{h}=\mathfrak{m} \oplus \mathfrak{a}$.

(ii) Clearly, the subspace $E=\mathcal{L}+\mathcal{L}^{\prime}$ is $\operatorname{ad}\left(\mathfrak{m} \oplus \mathfrak{a} \oplus \mathfrak{n}^{+}\right)$-invariant and contained in $\mathfrak{n}^{-} \oplus \mathfrak{h} \oplus$ $\mathfrak{n}^{+}$. Hence the result follows from Lemma 3.9.

Remark. - If $\mathfrak{h}$ is an ideal of $\mathfrak{m} \oplus \mathfrak{a}$ and

$$
V^{-}=\left\{v \in \mathfrak{n}^{-}: \forall k \in \mathbb{N},\left(\operatorname{ad}\left(\mathfrak{n}^{+}\right)\right)^{k}(v) \subset \mathfrak{n}^{-} \oplus \mathfrak{h} \oplus \mathfrak{n}^{+}\right\}
$$

$4^{\text {e }}$ SÉRIE - TOME $40-2007-\mathrm{N}^{\circ} 2$ 
then the above arguments show that $V^{-} \oplus \mathfrak{h} \oplus \mathfrak{n}^{+}$is the largest element of $\mathfrak{S}$. In this case $\mathcal{P}=V^{-} \oplus \mathfrak{m} \oplus \mathfrak{a} \oplus \mathfrak{n}^{+}$. This is valid, in particular, if $\mathfrak{h}$ is the Lie algebra of $H=\Gamma_{e}(T)$ (see Lemma 3.8). Then $\mathfrak{v}_{T}^{0} \subset V^{-} \subset \mathcal{P}$.

3.11. Proposition. - Let $T$ be a Zariski dense sub-semigroup of $G$ such that $\pi(e) \in L_{T}$ and $\pi^{\prime}(e) \in L_{T}^{\prime}$. We denote $H=\Gamma_{e}(T)$ and we call $\mathfrak{h}$ the Lie algebra of $H$.

Then $\mathfrak{a} \oplus \mathfrak{m}=\mathfrak{h}$ and consequently $H=M_{1} A$ where $M_{1}$ is a subgroup of finite index of $M$.

Proof. - We consider the set $\mathfrak{S}$ of subalgebra of $\mathfrak{g}$ defined in Lemma 3.10. $\mathfrak{S}$ is not empty: from Lemmas 3.8 and 3.9, $\mathfrak{S}$ contains the subalgebra of $\mathfrak{g}$ generated by $\mathfrak{v}_{T}^{0}$. We denote by $\mathcal{L}$ the largest element of $\mathfrak{S}$ and by $\mathcal{P}$ the standard parabolic subalgebra of $\mathfrak{g}$ defined in Lemma 3.10, $\mathcal{P}=\left(\mathfrak{m} \oplus \mathfrak{a} \oplus \mathfrak{n}^{+}\right)+\mathcal{L}$. Since $\mathfrak{h}$ is an ideal of $\mathfrak{m} \oplus \mathfrak{a}, \mathcal{L}=\mathcal{L}+\mathfrak{h}+\mathfrak{n}^{+}=\left(\mathcal{P} \cap \mathfrak{n}^{-}\right) \oplus \mathfrak{h} \oplus \mathfrak{n}^{+}$.

Since $\mathfrak{n}^{-}+\mathfrak{n}^{+}$generate $\mathfrak{g}$ and $\mathcal{L}+\mathfrak{h}+\mathfrak{n}^{+}$is a subalgebra, it suffices to prove that $\mathcal{P}=\mathfrak{g}$.

Assume that $\mathcal{P} \neq \mathfrak{g}$. We call $P$ the standard parabolic subgroup of $G$ having $\mathcal{P}$ for Lie-algebra. Let $\mu$ be a probability measure on $G$ such that $T_{\mu}=T$ and call $\nu$ the unique $\mu$-invariant measure on $F$. We know (Proposition 1.5) that there exists a sequence $\left(X_{n}\right)_{n \geqslant 0}$ of elements of $\mathfrak{n}^{-} \backslash \mathcal{P}$ converging to 0 such that, for any $n \in \mathbb{N}, \pi\left(\exp \left(X_{n}\right)\right) \in L_{T}$.

For any $s \in T \cap B$ and any $n$ large enough, we know that (see proof of Lemma 3.8),

$$
\eta(s) \exp \left(X_{n}\right)(\eta(s))^{-1}=\exp \left(\operatorname{Ad} \eta(s) X_{n}\right) \in N^{-} H N^{+} .
$$

We denote by $L$ the subgroup of $G$ generated by $\exp \mathcal{L}$. We consider $U \in \mathcal{P} \cap \mathfrak{n}^{-} \subset \mathcal{L}$. From properties of $\mathcal{L}$, the subgroup $M A N^{+}$normalize $L, \operatorname{Ad} \eta(s) U \in \mathcal{L}$ and $\exp (\operatorname{Ad} \eta(s) U) \in L \subset$ $\left(N^{-} \cap P\right) H N^{+}$so we obtain that: for any $s \in T \cap B, U \in \mathcal{P} \cap \mathfrak{n}^{-} \subset \mathcal{L}$ and any $n$ large enough,

$$
\eta(s) \exp \left(X_{n}\right) \exp (U)(\eta(s))^{-1}=\exp \left(\operatorname{Ad} \eta(s)\left(X_{n} \circ U\right)\right) \in N^{-} H N^{+} .
$$

Let $\|\cdot\|$ be a norm on $\mathfrak{n}^{-}$. For any $n \geqslant 0$, we choose $U_{n} \in \mathcal{P} \cap \mathfrak{n}^{-}$such that

$$
\left\|X_{n}\right\|^{\prime}=\min \left\{\left\|X_{n} \circ U\right\|: U \in \mathcal{P} \cap \mathfrak{n}^{-}\right\}=\left\|X_{n} \circ U_{n}\right\| \leqslant\left\|X_{n}\right\| .
$$

For $n$ large enough, we have

$$
\exp \left(\left\|X_{n}\right\|^{\prime} \operatorname{Ad} \eta(s)\left(\frac{X_{n} \circ U_{n}}{\left\|X_{n}\right\|^{\prime}}\right)\right) \in N^{-} H N^{+}
$$

and consequently, as in the proof of Lemma 3.8, for any cluster value $X$ of the sequence $\left(\frac{X_{n} \circ U_{n}}{\left\|X_{n}\right\|^{\prime}}\right)_{n \geqslant 0}$,

$$
\forall s \in T \cap B, \quad \operatorname{Ad} \eta(s)(X) \in \mathfrak{n}^{-} \oplus \mathfrak{h} \oplus \mathfrak{n}^{+} .
$$

Observe that $X$ does not belong to $\mathcal{P}$, because $\|X\|^{\prime}=1$. Proceeding as in the proof of Lemma 3.8, we prove that

$$
E_{X}=\bigcup_{k \in \mathbb{N}}\left\{\left(\operatorname{ad}\left(\mathfrak{m} \oplus \mathfrak{a} \oplus \mathfrak{n}^{+}\right)\right)^{k}(X)\right\} \subset \mathfrak{n}^{-} \oplus \mathfrak{h} \oplus \mathfrak{n}^{+} .
$$

From Lemma 3.9, we deduce that the Lie subalgebra $\mathcal{L}_{X}$ of $\mathfrak{g}$ generated by $E_{X}$ belongs to $\mathfrak{S}$. Then (Lemma 3.10) the Lie subalgebra of $\mathfrak{g}$ generated by $\mathcal{L}_{X}+\mathcal{L}$ belongs to $\mathfrak{S}$. This contradicts the maximality of $\mathcal{L}$. So we showed by contradiction that $\mathcal{P}=\mathfrak{g}$. 


\section{- Proofs of Theorem 1.9 and Corollary 1.10}

The first assertion follows from Lemma 3.7.

Then from Proposition 1.8 we know that $\Gamma_{g}(T)$ is independent of $g \in \pi^{\prime-1}\left(L_{T}^{\prime}\right)$. Hence it suffices to prove $\Gamma_{g}(T)=M_{T} A$ for $g$ such that $y=g^{-1} a m g$ is a proximal element of $G$, $\pi^{\prime}(g)=y^{+}$and $\pi\left(g^{-1}\right)=y^{-}$. Then we know (Proposition 1.8) that $\Gamma_{g}(T)$ is generated by elements $\gamma_{g}\left(t, y^{-}\right)=\gamma\left(g t g^{-1}, \pi(e)\right)$ with $t \in T$ such that $g t g^{-1} \in B$. We denote by $S$ the sub-semigroup $g T g^{-1} ; S$ contains a proximal element in $M A^{+}, \pi(e) \in L_{S}, \pi^{\prime}(e) \in L_{S}^{\prime}$ and $\gamma(S \cap B, \pi(e))$ generates the subgroup $\Gamma_{e}(S)=\Gamma_{g}(T)$. The relation $\Gamma_{e}(S)=A M_{T}$ follows from the above proposition.

From [8] (pp. 83-84) we know that the Zariski closure of $A$ in $G$ is the product of $A$ by a finite group $\Phi$ of involutions which is isomorphic to $(\mathbb{Z} / 2 \mathbb{Z})^{r}$, with $r=\operatorname{dim} A$. Also we know that $M / M_{0}$ is a factor group of $\Phi$. Hence $M / M_{0}$ is of the form $(\mathbb{Z} / 2 \mathbb{Z})^{r^{\prime}}$ with $r^{\prime} \leqslant r$ and since $M_{0} \subset M_{T}, M_{T} / M_{0}$ is of the form $(\mathbb{Z} / 2 \mathbb{Z})^{p}, p \leqslant r$.

The last assertion of the theorem is an immediate consequence of the equality $\Gamma_{g}(T)=$ $\operatorname{pr}^{-1}(\langle\operatorname{Spec} T\rangle)$.

From Theorem 1.9, we have:

$$
\langle\operatorname{Spec} T\rangle=A M_{T} /[M, M]=A M_{T} /[M, M] .
$$

It follows that $\langle\operatorname{Arg} T\rangle=M_{T} /[M, M]$ and the index of $\langle\operatorname{Arg} T\rangle$ in $M_{T} /[M, M]$ is equal to the index of $M_{T}$ in $M$. Clearly $M_{T}$ is the inverse image of $\langle\operatorname{Arg} T\rangle$ in $M$. From Lemma 3.7, we know that $[M, M]$ is connected, hence $[M, M] \subset M_{0}$. It follows that the identity component of $\langle\operatorname{Arg} T\rangle$ is the projection of $M_{0}$ in $M /[M, M]$, hence $M_{T} / M_{0}$ is isomorphic to $\langle\operatorname{Arg} T\rangle /\langle\operatorname{Arg} T\rangle_{0}$.

\section{- Proof of Theorem 2.4 and Corollary 2.5}

The assertions (i) and (ii) of Theorem 2.4 follows from the following proposition.

From Theorem 2.3 and the Ascoli theorem, one sees easily that for any continuous function $f$ on $X^{\rho}$, the sequence of functions $\left(\left(f+P_{\mu} f+\cdots+P_{\mu}^{n-1} f\right) / n\right)$ converges uniformly on $X^{\rho}$. We deduce that, for any $x \in X^{\rho}$, the sequence of probability $\left(\left(\delta_{e}+P_{\mu}(x, \cdot)+\cdots+P_{\mu}^{n-1}(x,).\right) / n\right)$, converges towards a probability $\lambda_{x}$ on $X^{\rho}$ which is $\mu$-invariant. Writing, $\lambda_{x}=\sum_{i=1}^{s} h_{i}(x) \sigma_{i}$, we obtain the basis $\left\{h_{i}: 1 \leqslant i \leqslant s\right\}$ of the vector space of $P_{\mu}$-invariant continuous functions on $X^{\rho}$. In fact, we have: $h_{i}(x)=\mathbb{P}\left[\left\{\lim _{n} d\left(L_{n} \cdot x, \operatorname{Supp}\left(\sigma_{i}\right)\right)=0\right\}\right]$ (see [29], Section 2).

3.12. Proposition. - Let $G$ be an algebraic Zariski connected real semi-simple group. Let $\mu$ be a probability measure on $G$ such that $T_{\mu}$ is a Zariski dense sub-semigroup of $G$. Let $\sigma$ be an extremal $\mu$-invariant probability measure on $X^{\rho}$. We denote by $\lambda$ the normalized Haar-measure on the closed subgroup $C_{T_{\mu}}=\overline{\rho\left(A M_{T_{\mu}}\right)}$ of $C$.

Then we have:

$$
\sigma=\int_{F} \delta_{x} \otimes m(x) \lambda \nu(d x) ;
$$

where $m$ is a Borel map from $F$ to $C$ such that, for any $t \in T_{\mu}$ and $\nu$-almost every $x \in F$,

$$
m(t x) C_{T_{\mu}}=\rho(\gamma(t, x)) m(x) C_{T_{\mu}} .
$$

Consequently, the finite set of extremal $\mu$-invariant probability measures on $X^{\rho}$ is equal to $\left\{\sigma c: c \in C / C_{T_{\mu}}\right\}$. If $\pi^{\prime}(e) \in L_{T}^{\prime}$ then $\sigma=\hat{\nu} \otimes \lambda$.

$4^{\mathrm{e}}$ SÉRIE - TOME $40-2007-\mathrm{N}^{\circ} 2$ 
Proof. - First of all, the definition of $M_{T_{\mu}}$ and $C_{T \mu}$ implies that the formula (1) defines a $\mu$-invariant probability measure on $X^{\rho}$.

The sequence of probability measures $\left(R_{n} \cdot \sigma=Y_{1} \cdots Y_{n} \cdot \sigma\right)$ is a probability-valued martingale with respect to the filtration $\left(\mathcal{F}_{n}=\sigma\left(Y_{1}, \ldots, Y_{n}\right)\right)$; hence it converges, on a Borel subset $\Omega_{0}$ of $\Omega$ with $\mathbb{P}$-measure 1 , to a probability measure on $X^{\rho}$.

Let $k \in K$. The sequence of probability measures $\left(k R_{n} \cdot \sigma\right)$ converges on $\Omega_{0}$. As $\mathbf{u}\left(k R_{n}\right)$ converges, $\mathbb{P}$-a.e., to $\hat{Z}(k, \cdot)$, the sequence of probability measures $\mathbf{b}\left(k R_{n}\right) \mathbf{k}\left(k R_{n}\right) \cdot \sigma$ converges, $\mathbb{P}$-a.e., to a probability measure $\beta(k, \cdot)$.

We call $\Omega_{k}$ the Borel subset of $\Omega_{0}$ formed with elements $\omega$ which satisfy the assertions (i) and (ii) of Theorem 3.4, for $g=k$. Let $\omega \in \Omega_{k}$ and $\varphi(n)$ be an increasing sequence of natural integers such that $\mathbf{k}\left(k R_{\varphi(n)}(\omega)\right)$ converges to $k^{\prime} \in K$. For any $x \in \pi\left(k^{\prime-1} N^{-}\right)$and $n$ large enough, $\mathbf{k}\left(k R_{\varphi(n)}(\omega)\right) \cdot x$ belongs to $\pi\left(N^{-}\right)$and converges to $k^{\prime} \cdot x \in \pi\left(N^{-}\right)$. Then $\mathbf{b}\left(k R_{\varphi(n)}(\omega)\right) \mathbf{k}\left(k R_{\varphi(n)}(\omega)\right) \cdot x$ converges to $\pi(e)$. It follows that

$$
\beta(k, \omega)=\delta_{\pi(e)} \otimes \lambda_{k, \omega} \quad \text { and } \quad k R_{n}(\omega) \cdot \sigma \underset{n \rightarrow+\infty}{\longrightarrow} \hat{Z}(k, \cdot) \cdot \beta(k, \omega)=\delta_{k \cdot Z(\omega)} \otimes \lambda_{k, \omega} ;
$$

where $\lambda_{k, \omega}$ is a probability measure on $C$.

From the equality $k R_{n}=\mathbf{u}\left(k Y_{1}\right) \mathbf{b}\left(k Y_{1}\right) \mathbf{k}\left(k Y_{1}\right) R_{n-1} \circ \theta$, it follows that,

$$
\forall k \in K, \forall \omega \in \Omega_{k}, \quad \lambda_{k, \omega}=\rho\left(\mathbf{b}\left(k Y_{1}(\omega)\right)\right) \lambda_{\tilde{\theta}(k, \omega)},
$$

where $\tilde{\theta}$ is the map from $K \times \Omega$ to itself, defined by: $\tilde{\theta}(k, \omega)=\left(k \cdot Y_{1}(\omega), \theta(\omega)\right)$.

We observe also that for any $m \in M, \Omega_{m k}=\Omega_{k}, \mathbf{u}(m k g)=m \mathbf{u}(k g) m^{-1}, \mathbf{b}(m k g)=\mathbf{b}(k g)$, $\mathbf{k}(m k g)=m \mathbf{k}(k g)$ and consequently,

$$
\forall \omega \in \Omega_{k}, \forall k \in K, \quad \lambda_{m k, \omega}=\rho(m) \lambda_{k, \omega} .
$$

For $k \in K$, we call $\sigma_{k}$ the probability on $C$ defined by, for any bounded Borel function $f$ on $C$,

$$
\sigma_{k}(f)=\mathbb{E}\left[\lambda_{k, \cdot}(f)\right]=k \cdot \sigma\left(1_{F} \otimes f\right)=\int_{Q_{k}} f(\rho(\gamma(k, x)) c) \sigma(d x, d c),
$$

where $Q_{k}=\left(\pi\left(N^{-}\right) \cap k^{-1} \cdot \pi\left(N^{-}\right)\right) \times C$ (observe that the projection of the probability measure $\sigma$ on $F$ is the probability measure $\nu$ and therefore, $\left.\forall g \in G, \sigma\left(g \cdot \pi\left(N^{-}\right) \times C\right)=1\right)$.

Let $\kappa$ be an extremal $\mu$-invariant probability measure on $K$, identified to $N^{-} A \backslash G ; \kappa * \mu=\kappa$. It is well-known that the dynamical system $(K \times \Omega, \tilde{\theta}, \kappa \times \mathbb{P})$ is ergodic.

- Abelian case. We suppose that the group $C$ is abelian. For any character $\chi$ on $C$ we define the function $f_{\chi}$ on $C$ by: $\forall k \in K, f_{\chi}(k)=\sigma_{k}(\chi)$. We observe that, from equation (4),

$$
\forall(m, k) \in M \times K, \quad f_{\chi}(m k)=\chi(\rho(m)) f_{\chi}(k) .
$$

On the open set $\left\{(k, x) \in K \times F: x \quad\right.$ and $\left.\quad k \cdot x \in \pi\left(N^{-}\right)\right\}$the function $\gamma(k, x)$ is continuous; therefore the function $f_{\chi}$ is continuous. From (3) and (5) we deduce that $f_{\chi}$ satisfies the functional equation:

$$
\forall k \in K, \quad f_{\chi}(k)=\int_{G} \chi(\rho(\mathbf{b}(k g))) f_{\chi}(k \cdot g) d \mu(g) .
$$


From (7) we obtain, for any $k \in K,\left|f_{\chi}(k)\right| \leqslant \int_{G}\left|f_{\chi}(k \cdot g)\right| \mu(d g)$ and therefore for $\kappa$-almost every $k,|f(k)|=\int_{G}|f(k \cdot g)| \mu(d g)$. As $\kappa$ is extremal this implies that $\left|f_{\chi}\right|$ is constant $\kappa$-a.e. and consequently, by continuity, $\left|f_{\chi}\right|$ is constant on $\operatorname{Supp}(\kappa)$.

Suppose that $f_{\chi}$ is not equal to the null function on $K$; we can assume that $|f|=1$ on $\operatorname{Supp}(\kappa)$. We obtain that, for any $k \in \operatorname{Supp}(\kappa)$,

$$
\int_{G} \chi(\rho(\mathbf{b}(k g))) \frac{f_{\chi}(k \cdot g)}{f_{\chi}(k)} \mu(d g)=1
$$

and consequently, for any $(k, g) \in \operatorname{Supp}(\kappa) \times T_{\mu}$,

$$
\chi(\rho(\mathbf{b}(k g))) \frac{f_{\chi}(k \cdot g)}{f_{\chi}(k)}=1 .
$$

Let $g \in T_{\mu}^{\text {prox }}$ and write $g=y_{m a y}^{-1}$ with $y \in G$ and $m a \in M A^{+}$. We write $y^{-1}=$ $u b k^{\prime} \in N^{-} A K$. We have $k^{\prime} g=b^{-1} u^{-1} m a u b k^{\prime}$; hence $\mathbf{b}\left(k^{\prime} g\right)=a$ and $\mathbf{k}\left(k^{\prime} t\right)=m k^{\prime}$. As $\pi^{\prime}\left(k^{\prime}\right)=\pi^{\prime}\left(y^{-1}\right) \in \operatorname{Supp}\left(\nu^{\prime}\right)$ and the projection of $\kappa$ on $F^{\prime}=M \backslash K$ is $\nu^{\prime}$, there exists $m_{0} \in M$ such that $k=m_{0} k^{\prime} \in \operatorname{Supp}(\kappa)$.

From the above equality, we deduce $\chi(\rho(a m))=1$.

We have proved that $\chi \circ \rho\left(\operatorname{pr}^{-1}\left\langle\operatorname{Spec} T_{\mu}\right\rangle\right)=\{1\}$. From Theorem 1.9 it follows that $\chi \circ \rho\left(A M_{T_{\mu}}\right)=\{1\}$.

In one hand, we deduce that, for any character $\chi$ on $C$

$$
\forall k \in \operatorname{Supp}(\kappa), \forall g \in \operatorname{Supp}(\mu), \quad \sigma_{k}(\chi)=\sigma_{k \cdot g}(\chi) .
$$

Hence, for any $(k, g) \in \operatorname{Supp}(\kappa) \times T_{\mu}, \sigma_{k}=\sigma_{k \cdot g}$. On the other hand we deduce that, for any character $\chi$ on $C$,

$$
\forall k \in \operatorname{Supp}(\kappa), \forall g \in \operatorname{Supp}(\mu), \quad \sigma_{k}(\chi)=\left(\rho(\mathbf{b}(k g)) \sigma_{k \cdot g}\right)(\chi) .
$$

Hence, $\sigma_{k}$ is invariant by the elements of $\rho(A)$.

From (3), for $k \in \operatorname{Supp}(\kappa)$ and $\omega \in \Omega_{k}$, we have

$$
\lambda_{k, \omega}=\rho\left(\mathbf{b}\left(k Y_{1} \cdots Y_{n}\right)\right) \lambda_{k \cdot Y_{1}(\omega) \cdots Y_{n}(\omega), \theta^{n}(\omega)} .
$$

Therefore

$$
\mathbb{E}\left[\lambda_{k, \mid} \mid \mathcal{F}_{n}\right]=\rho\left(\mathbf{b}\left(k Y_{1} \cdots Y_{n}\right)\right) \sigma_{k \cdot Y_{1} \cdots Y_{n}}=\sigma_{k}, \quad \mathbb{P} \text {-a.e. }
$$

and consequently $\lambda_{k, \cdot}=\sigma_{k}, \mathbb{P}$-a.e.

Then, from (4) and Theorem 1.9 we deduce that $\sigma_{k}$ is invariant by the elements of $\rho\left(\Gamma_{k}\left(T_{\mu}\right)\right)=\rho\left(A M_{T_{\mu}}\right)$. We can write $\sigma_{k}=\lambda * \alpha$ where $\alpha$ is a probability measure on $C / C_{T_{\mu}}$. On the other hand, from above, the measure $\hat{\nu} \otimes(\lambda * \alpha)$ is $\mu$-invariant for any probability measure $\alpha$ on $C / C_{T_{\mu}}$. It follows that if $\sigma$ is extremal then $\alpha$ is a Dirac measure, hence $\sigma$ has the required form.

Finally from (2) we obtain, for $k \in \operatorname{Supp}(\kappa)$,

$$
\sigma=\int_{F} \delta_{x} \otimes(\rho(\gamma(k, x)))^{-1} c \lambda d \nu(x)
$$

$4^{e}$ SÉRIE - TOME $40-2007-\mathrm{N}^{\circ} 2$ 
for an element $c \in C$. Setting $m(x)=(\rho(\gamma(k, x)))^{-1} c$ we have for any $t \in T_{\mu}$,

$$
\begin{aligned}
\rho(\gamma(t, x)) m(x) C_{T_{\mu}} & =\rho\left(\gamma(t, x) \gamma\left(k^{-1}, k \cdot x\right)\right) c C_{T_{\mu}} \\
& =\rho\left((\gamma(k, t \cdot x))^{-1} \gamma\left(k t k^{-1}, k \cdot x\right)\right) c C_{T_{\mu}} \\
& =\rho\left((\gamma(k, t \cdot x))^{-1}\right) c C_{T_{\mu}}=m(t \cdot x) C_{T_{\mu}} .
\end{aligned}
$$

- General case. We prove that

$$
\forall m \in[M, M], \forall k \in K, \quad \rho(m) \cdot \sigma_{k}=\sigma_{k} .
$$

So that we can identify $\sigma_{k}$ with a probability on the abelian group $C / \rho([M, M])$. Then we conclude using the first case.

From (3) it follows that, for any $k \in K$ and $\omega \in \Omega_{k}, \overline{\rho(A)} \lambda_{k, \omega}=\overline{\rho(A)} \lambda_{\tilde{\theta}(k, \omega)}$. From the ergodicity of the dynamical system $(K \times \Omega, \tilde{\theta}, \kappa \times \mathbb{P})$, We deduce that there exists a probability $\lambda$ on $C$ such that, for $\kappa \otimes \mathbb{P}$-almost every $(k, \omega) \in K \times \Omega, \overline{\rho(A)} \lambda_{k, \omega}=\overline{\rho(A)} \lambda$. Hence, for $\kappa \otimes \mathbb{P}$-almost every $(k, \omega) \in K \times \Omega$, there exists $a(k, \omega) \in \overline{\rho(A)}$ with

$$
\lambda_{k, \omega}=a(k, \omega) \lambda
$$

Since $k R_{n} \cdot \sigma=\left(k Y_{1} k^{-1}\right) k R_{n-1} \circ \theta$, this convergence says us that, for $k \in K$ and $\omega \in \Omega_{k}$ such that $k \cdot Z(\omega) \in \pi\left(N^{-}\right)$and $k \cdot Z(\theta(\omega)) \in \pi\left(N^{-}\right)$,

$$
\begin{aligned}
\delta_{k \cdot Z(\omega)} \otimes \lambda_{k, \omega} & =\left(k Y_{1}(\omega) k^{-1}\right) \cdot\left(\delta_{k \cdot Z(\theta(\omega))} \otimes \lambda_{k, \theta(\omega)}\right) \\
& =\delta_{k \cdot Z(\omega)} \otimes \rho \circ \gamma\left(k Y_{1}(\omega) k^{-1}, k \cdot Z(\theta(\omega))\right) \lambda_{k, \theta(\omega)} .
\end{aligned}
$$

We call $\Pi$ the closed subgroup of $C$ defined by: $\Pi=\{c \in C: c \lambda=\lambda\}$. From (5) and (6), it follows that, for $\kappa \otimes \mathbb{P}$-almost every, $(k, \omega)$,

$$
a^{-1}(k, \omega) \rho \circ \gamma\left(k Y_{1}(\omega) k^{-1}, k \cdot Z(\theta(\omega))\right) a(k, \theta(\omega)) \in \Pi .
$$

It follows that for $(\kappa \otimes \mathbb{P}) \otimes(\kappa \otimes \mathbb{P})$-almost every $\left((k, \omega),\left(k^{\prime}, \omega^{\prime}\right)\right) \in(K \times \Omega)^{2}$, $\left[\rho \circ \gamma\left(k Y_{1}(\omega) k^{-1}, k \cdot Z(\theta(\omega))\right), \rho \circ \gamma\left(k^{\prime} Y_{1}\left(\omega^{\prime}\right) k^{\prime-1}, k^{\prime} \cdot Z\left(\theta\left(\omega^{\prime}\right)\right)\right)\right]$ belongs to $\Pi$ and therefore from Theorem $1.9\left[\rho\left(A M_{T_{\mu}}\right), \rho\left(A M_{T_{\mu}}\right)\right]=\left[\rho\left(M_{T_{\mu}}\right), \rho\left(M_{T_{\mu}}\right)\right]$ belongs to $\Pi$. Since $[M, M]$ is semi-simple and connected, we have $[M, M]=\left[M_{0}, M_{0}\right]$. We deduce that $\rho([M, M]] \subset \Pi$.

3.13. COROLLARY. - Let $G$ be an algebraic Zariski connected real semi-simple group. Let $\mu$ be a probability measure on $G$ such that $T_{\mu}$ is a Zariski dense sub-semigroup of $G$. Let $\kappa$ be an extremal $\mu$-invariant probability measure on $K=G / A N^{+}$. Then

$$
\kappa=\int_{F} \delta_{x} \otimes m(x) \lambda \nu(d x),
$$

where $m$ is a Borel map from $F$ to $M$ such that, for any $t \in T_{\mu}$ and $\nu$-almost every $x \in F$,

$$
m(t x) M_{T_{\mu}}=\operatorname{proj}_{M} \circ \gamma(t, x) m(x) M_{T_{\mu}} .
$$

The set of extremal $\mu$-invariant probability measures on $K=G / A N^{+}$is $\left\{\kappa m: m \in M / M_{T_{\mu}}\right\}$.

For any $k \in \operatorname{Supp}(\kappa)$, the extremal $k^{-1} \mu k$-invariant probability measures are $\left\{\left(k^{-1} \cdot \nu \otimes \lambda\right) c\right.$ : $\left.c \in C / C_{T_{\mu}}\right\}$. In particular, if $\pi^{\prime}(e) \in L_{T}^{\prime}, \hat{\nu} * \lambda$ is extremal. 
Proof. - The first assertion is obtained when $C=M$ and $\rho$ is the projection of $A M N^{+}$on $M$. We observe that $e \in \operatorname{Supp}\left(k^{-1} \kappa\right)$; the second assertion is clear from formula (3), with $k=e$.

\section{- Cyclic classes and proof of Theorem 2.6}

3.14. Definition (see [29]). - Let $\sigma$ be an extremal $\mu$-invariant probability on $X^{\rho}$. We say that two elements $x$ and $y$ of $\operatorname{Supp}(\sigma)$ are equivalent if $h(x)=h(y)$ for any continuous eigenfunction $h$ with eigenvalue of modulus one of the operator $P_{\mu}$. Any equivalence class is closed and called "a cyclic subclass" of $\operatorname{Supp}(\sigma)$. We denote by $\mathcal{C}_{\sigma}$ the set of cyclic subclasses of $\operatorname{Supp}(\sigma)$. The following is an adaption of the general result of [29].

3.15. Proposition. - We assume that $\mu$ satisfies hypothesis $(\mathrm{H})$ and we denote by $\mathcal{E}$ the set of extremal $\mu$-invariant probability measures on $X^{\rho}$. Let $\sigma$ be a probability measure in $\mathcal{E}$.

(i) Let $h$ be a continuous function on $\operatorname{Supp}(\sigma)$ verifying $P_{\mu} h=\lambda h$ with $|\lambda|=1$. Then we have: $\forall(g, x) \in \operatorname{Supp}(\mu) \times \operatorname{Supp}(\sigma), h(g \cdot x)=\lambda h(x)$. It follows that, for any cyclic subclass $\varsigma$ of $\operatorname{Supp}(\sigma), \operatorname{Supp}(\mu) \cdot \varsigma$ is included in a cyclic subclass of $\operatorname{Supp}(\sigma)$. In other words, $\operatorname{Supp}(\mu)$ induced a transformation $\tau$ on the space $\mathcal{C}_{\sigma}$.

(ii) We can endow the space $\mathcal{C}_{\sigma}$ with a distance $\delta$ for which $\tau$ is an isometry. We consider the isometry group of $\mathcal{C}_{\sigma}$ and we call $L$ the closed abelian subgroup, which is also the closed abelian sub-semigroup, generated by $\tau$. The group $L$ acts transitively on $\mathcal{C}_{\sigma}$.

(iii) The projection on $F$ of any $\varsigma \in \mathcal{C}_{\sigma}$ is equal to $\operatorname{Supp}(\nu)$.

(iv) For any $\varsigma \in \mathcal{C}_{\sigma}$ and $c \in C_{T_{\mu}}$, $\varsigma \cdot c \in \mathcal{C}_{\sigma}$. This action of $C_{T_{\mu}}$ is transitive and there exists $c_{0} \in C_{T_{\mu}}$ so that, for any $\varsigma \in \mathcal{C}_{\sigma}, \tau \varsigma=\varsigma \cdot c_{0}$. For any function $h$ as in (i), there exists a character $\chi$ of $C_{T_{\mu}}$ such that $\forall m \in C_{T_{\mu}}, h(\cdot m)=\chi(m) h$. If we call $H_{\sigma}$ the normal closed subgroup of $C_{T_{\mu}}$ which stabilizes any cyclic subclass of $\mathcal{C}_{\sigma}$, the quotient group $C_{T_{\mu}} / H_{\sigma}$ is generated by the image of $c_{0}$ and can be identified to the group $L$.

Proof. - The assertions (i) and (ii) are proved in [29] (see Sections 4.5 and 4.6). The first one follows from the equality

$$
\int_{X^{\rho}} \int_{G}|h(g \cdot x)-\lambda h(x)|^{2} \mu(d g) \sigma(d u)=0 .
$$

For the second assertion, we remark that the union of the spaces of continuous eigenfunction with eigenvalue of modulus 1 is separable for the uniform topology. We take a dense sequence $\left(h_{n}\right)_{n \geqslant 0}$ and define

$$
\forall \varsigma, \varsigma^{\prime} \in \mathcal{C}_{\sigma}, \quad \delta\left(\varsigma, \varsigma^{\prime}\right)=\sum_{k \geqslant 0} 2^{-k} \frac{\left|h_{k}(\varsigma)-h_{k}\left(\varsigma^{\prime}\right)\right|}{\left\|h_{k}\right\|} .
$$

For any cyclic subclass $\varsigma$ of $\operatorname{Supp}(\sigma)$, the closure of the union of cyclic subclasses $\bigcup_{n \geqslant 0} \tau^{n} \varsigma$ is invariant by $T_{\mu}$ and contains the support of a probability $\mu$-invariant measure. As $\sigma$ is the unique probability $\mu$-invariant measure on $\operatorname{Supp}(\sigma)$, it follows that this closure is equal to $\operatorname{Supp}(\sigma)$.

(iii) From Corollary 3.6, it follows that, for any sequence $\left(x_{n}\right)_{n \geqslant 0}$ of elements of $F$, the sequence of probability measures $\left(\mu^{n} \star \delta_{x_{n}}\right)_{n \geqslant 0}$ converges weakly towards the unique $\mu$ invariant probability $\nu$ on $F$.

Then, it is well-known that, for any compact subset $C$ of $F$, we have

$$
\nu(C) \geqslant \limsup _{n \rightarrow+\infty} \mu^{n} \star \delta_{x_{n}}(C) .
$$

$4^{\text {e }}$ SÉRIE - TOME $40-2007-\mathrm{N}^{\circ} 2$ 
Let $\varsigma$ be a cyclic subclass of $\operatorname{Supp}(\sigma)$. We denote by $\varpi(\varsigma)$ its projection in $F$. We have $\nu(\varpi(\varsigma)) \geqslant \limsup _{n \rightarrow \infty} \mu^{n} \star \delta_{x_{n}}(\varpi(\varsigma))$. But if we choose a sequence $\left(x_{n}\right)_{n \geqslant 0}$ such that $x_{n} \in \varpi\left(\tau^{-n}(\varsigma)\right)$, we have $\mu^{n} \star \delta_{x_{n}}(\varpi(\varsigma))=1$ and consequently $\nu(\varpi(\varsigma))=1$.

(iv) We know that, for any $x \in \operatorname{Supp}(\sigma), \overline{T_{\mu} \cdot x}=\operatorname{Supp}(\sigma)$. From (i) it follows that any $P_{\mu}$-invariant continuous function on $\operatorname{Supp}(\sigma)$ is constant. Let $h$ be a continuous function on $X^{\rho}$ such that $P_{\mu} h=\lambda h$ with $|\lambda|=1$. The function $|h|$ satisfies $P_{\mu}|h| \geqslant|h|$; consequently $P_{\mu}|h|=|h| \sigma$-a.e. and, by continuity, $|h|$ is $P_{\mu}$-invariant on $\operatorname{Supp}(\sigma)$. Hence $|h|$ is constant on $\operatorname{Supp}(\sigma)$ and we can suppose that $\forall x \in \operatorname{Supp}(\sigma),|h(x)|=1$. Now we observe that the $C$-right translations preserve the space of continuous eigenfunction with eigenvalue $\lambda$ of the operator $P_{\mu}$. For any $m \in C_{T_{\mu}}$, the quotient function $\frac{h(\cdot m)}{h}$ is $P_{\mu}$ invariant and thus constant on $\operatorname{Supp}(\sigma)$; therefore, there exists a character $\chi$ on $C_{T_{\mu}}$ such that $h(\cdot m)=\chi(m) h$. We deduce that $H_{\sigma}=\operatorname{Ker} \chi$ is a closed normal subgroup of $C_{T_{\mu}}$ stabilizing any cyclic subclasses. From (iii), it follows that $C_{T_{\mu}}$ acts transitively on $\mathcal{C}_{\sigma}$. If $c_{0}$ is one element of $C_{T_{\mu}}$ such that $\tau \cdot \varsigma=\varsigma \cdot c_{0}$, then the quotient group $C_{T_{\mu}} / H_{\sigma}$ is generated by the image of $c_{0}$.

3.16. THEOREM. - Let $G$ be an algebraic Zariski connected real semi-simple group and $\mu$ be a probability measure on $G$. We assume that the Zariski closure of the closed sub-semigroup $T_{\mu}$ generated by the support of $\mu$ is equal to $G$. Then the support $\operatorname{Supp}(\sigma)$ of any extremal $\mu$-invariant probability $\sigma$ on $X^{\rho}$ either has no cyclic subclasses or splits in two cyclic subclasses. In the first case, the peripheral spectrum of $P_{\mu}$ is $\{1\}$ and in the last case, the peripheral spectrum of $P_{\mu}$ is $\{-1,1\}$.

Proof. - From Corollary 3.8, replacing the probability measure $\mu$ by a conjugate, we can assume that $\operatorname{Supp}(\sigma)=\operatorname{Supp}(\nu) \times C_{T_{\mu}}$ and $e$ belongs to the support of an extremal $\mu$-invariant probability measure $\kappa^{\prime}$ of $K=N^{-} A \backslash G$. Hence $e$ is a recurrent point for the right random walk on $K$.

For any $g \in G$ and $u \in N^{-}$such that $g u \in B$, we have

$$
g \cdot(\pi(u), e)=(g \cdot \pi(u), \rho(\gamma(g, \pi(u))))
$$

From Proposition 3.10, for any $\varsigma \in \mathcal{C}_{\sigma}$ and $t \in T_{\mu}$, t. $\varsigma$ is included in a cyclic subclass of $\operatorname{Supp}(\sigma)$ and for any continuous function such that $P_{\mu} h=\lambda h$ with $|\lambda|=1$, we have:

$$
\forall t \in T_{\mu}, \forall x \in \operatorname{Supp}(\sigma), \quad h(t . x)=\theta(t) h(x)
$$

with $\theta(t)=\lambda^{n}$ if $t$ belongs to $\operatorname{Supp}\left(\mu^{* n}\right)$. It follows that $\theta$ is a character on $T_{\mu}$ (i.e. a continuous function on $T_{\mu}$ such that $\forall s, t \in T_{\mu}, \theta(s t)=\theta(s) \theta(t)$ ).

For any $t \in T_{\mu}$ and $u \in S_{\hat{\nu}}$ such that $t u \in B$, we have, using the assertion (iv) of Proposition 3.15,

$$
\begin{aligned}
\theta(t) & =\frac{h(t \cdot(\pi(u), e))}{h(\pi(u), e)}=\frac{h(t \cdot \pi(u), \rho \circ \gamma(t, \pi(u)))}{h(\pi(u), e)} \\
& =\chi \circ \rho \circ \gamma(t, \pi(u)) \frac{h(t \cdot \pi(u), e)}{h(\pi(u), e)} .
\end{aligned}
$$

Considering the continuous function $\psi$ on $\pi\left(N^{-}\right)$defined by:

$$
\forall x \in \pi\left(N^{-}\right), \quad \psi(x)=h(x, e),
$$


we obtain, for any $t \in T_{\mu}$ and $x \in \operatorname{Supp}(\nu) \cap \pi\left(N^{-}\right)$such that $t . x \in \operatorname{Supp}(\nu) \cap \pi\left(N^{-}\right)$,

$$
\theta(t)=\frac{\psi(t \cdot x)}{\psi(x)} \chi \circ \rho(\gamma(t, x))
$$

We consider a sequence $\left(t_{n}\right)_{n \geqslant 0}$ of elements of $T_{\mu}$ such that: $\mathbf{u}\left(t_{n}\right)$ converges toward $z \in N^{-}$; $\forall \alpha \in \Delta_{+}, \varphi_{\alpha}\left(\mathbf{b}\left(t_{n}\right)\right)$ converges towards 0 and $\mathbf{k}\left(t_{n}\right)$ converges towards $e$, since $\pi^{\prime}(e) \in L_{T}^{\prime}$. Any typical trajectory of the right random walk has a subsequence verifying such properties.

For any $x \in \pi\left(N^{-}\right)$, the sequence $t_{n} \cdot x$ converges to $z$ and belongs to $B$ for $n$ sufficiently large. The sequence $\gamma\left(t_{n}, x\right)$ converges to $\gamma(e, x)=e$. Hence taking, if necessary, a subsequence, we can suppose that $\theta\left(t_{n}\right)$ converges. From the above equality, it follows that the function $\psi$ is constant on $\pi\left(N^{-}\right) \cap \operatorname{Supp}(\nu)$ and therefore

$$
\forall t \in T_{\mu}, \forall x \in \operatorname{Supp}(\nu) \cap N^{-}, \quad t x \in B, \quad \theta(t)=\chi \circ \rho(\gamma(t, x)) .
$$

As $\pi(e) \in \operatorname{Supp}(\nu)$, we have also

$$
\forall t \in T_{\mu} \cap B, \quad \theta(t)=\chi \circ \rho(\gamma(t, e))
$$

and therefore

$$
\forall t \in T_{\mu} \cap B, \forall x \in \operatorname{Supp}(\nu) \cap N^{-}, \quad t x \in B, \quad \chi \circ \rho\left(\gamma^{-1}(t, e) \gamma(t, x)\right)=1 .
$$

From Proposition 3.11, then we have $\chi \circ \rho\left(A M_{1}\right)=1$ where $M_{1}$ is a closed subgroup of $M$ containing the unit component of $M$. It follows that $H_{\sigma}$ contains $\rho(A)$ and $C_{T_{\mu}} / H_{\sigma}$ is a finite group. We denote by $\gamma_{M}(t, x)$ the projection on $M$ of $\gamma(t, x)$. Then, we have

$$
\forall t \in T_{\mu}, \forall x \in \operatorname{Supp}(\nu) \cap N^{-}, \quad t x \in B, \quad \theta(t)=\chi \circ \rho\left(\gamma_{M}(t, x)\right) .
$$

Now we know that $g \in B \rightarrow\left(\gamma_{M}(g, \pi(e))\right)^{2}$ is a rational map from $G$ to $M$. The restriction of $\chi \circ \rho$ to $M$ is a character $\chi_{M}$ on $M$. It follows that for any $x \in \pi\left(N^{-}\right) \cap \operatorname{Supp}(\nu)$, the map $g \in G \longrightarrow \chi_{M}^{2}\left(\gamma_{M}(g, x)\right)$ is a rational map on $G$. As $T_{\mu}$ is Zariski dense in $G$, the relation

$$
\chi_{M}^{2}\left(\gamma_{M}(s t, x)\right)=\chi_{M}^{2}\left(\gamma_{M}(s, x)\right) \chi_{M}^{2}\left(\gamma_{M}(t, x)\right)
$$

valid for any $(s, t) \in T_{\mu}^{2}$ such that $s x, t x, s t x \in B$, remains valid for any $(y, g) \in G$ such that $y x, g x, y g x \in B$. Since $G$ has only trivial characters, it follows that, for any $t \in T_{\mu}, \theta^{2}(t)=1$. Since $\theta(t)=\lambda^{n}$ for $t \in \operatorname{Supp}\left(\mu^{* n}\right)$, we obtain $\lambda=1$ or $\lambda=-1$.

Remark. - More generally, the proof shows that, for any characters $\chi$ on $A M$ and $\theta$ on $T_{\mu}$, the cohomological equation:

$$
\forall(t, x) \in T_{\mu} \times \operatorname{Supp}(\nu) \cap \pi\left(N^{-}\right) \quad \text { with } t . x \in \operatorname{Supp}(\nu) \cap \pi\left(N^{-}\right), \theta(t)=\frac{\psi(t \cdot x)}{\psi(x)} \chi(\gamma(t, x))
$$

has only continuous solutions $\psi$ on $\operatorname{Supp}(\nu)$ if $\theta^{2} \equiv 1$. In particular, if $\chi(M)=1$ we have solutions only for $\theta \equiv 1$ and then any solution $\psi$ is constant.

\section{- Proof of Theorem 2.6}

The first assertion of Theorem 2.6 follows from Proposition 3.15 and Theorem 3.16.

$4^{\text {e }}$ SÉRIE - TOME $40-2007-\mathrm{N}^{\circ} 2$ 
In general theory of Markov chains, it is well known that the behavior of $P_{\mu}^{n} f$ is strongly connected to "the space of $P_{\mu}$-asymptotic functions"; i.e. sequence of functions $\left(h_{n}\right)_{n \in \mathbb{N}}$ on $X^{\rho}$, such that $P_{\mu} h_{n+1}=h_{n}$.

Let $f$ be a continuous function on $X^{\rho}$. As the operator $P_{\mu}$ is equicontinuous, there exists a sequence of positive integers such that, for all $k \in \mathbb{N}$, the sequence of functions $P^{\varphi(n)-k} f$ converges uniformly on $X^{\rho}$. This convergence gives us a $P_{\mu}$-asymptotic function $\left(h_{k}\right)$ for which the family of functions $\left\{h_{k}: k \in \mathbb{N}\right\}$ is equicontinuous. For any eigenfunction $f$ of modulus 1 of $P_{\mu}, P_{\mu} f=\lambda f$, the sequence of functions $h_{n}=\lambda^{-n} f$ is a $P_{\mu}$-asymptotic function with the required property.

For an equicontinuous Markov operator $P$ on a compact space, it is proved in [29] that the vector space of $P$-asymptotic continuous functions $\left(h_{n}\right)_{n \in \mathbb{N}}$ such that the family of functions $\left\{h_{n}: n \in \mathbb{N}\right\}$ is equicontinuous coincides (via natural identification) with the closure of the vector space generated by the continuous eigenfunctions of modulus 1 of $P_{\mu}$.

If the peripheral spectrum of $P_{\mu}$ is $\{1\}$, for any $k \in \mathbb{N}, h_{k}=h$, with $P_{\mu} h=h$. From Proposition 3.15, this function is constant, and necessarily equal to $\sigma_{i}(f)$, on each $\operatorname{Supp}\left(\sigma_{i}\right)$, $1 \leqslant i \leqslant s$. Then $h=\sum_{i=1}^{s} \sigma_{i}(f) h_{\sigma_{i}}$ does not depend on the sequence $(\varphi(n))$. Hence the assertion (ii) of Theorem 2.6.

When the peripheral spectrum of $P_{\mu}$ is $\{-1,1\}$, assertion (iii) of Theorem 2.6 follows from Proposition 3.15 and the fact that the peripheral spectrum of $P_{\mu^{* 2}}$ is $\{1\}$.

\section{The algebraic hull of the cocycle defined by $\mu$}

Let $\Gamma$ be a locally compact group acting on a standard probability space $(X, \mathfrak{B}(X), m)$. Let $G$ be an $\mathbb{R}$-algebraic group and $\rho$ a $G$-valued cocycle on the dynamical system $(X, \mathfrak{B}(X), m, \Gamma)$; i.e. $\rho: \Gamma \times X \longrightarrow G$ with

$$
\forall \gamma_{1}, \gamma_{2} \in \Gamma, \forall x \in X, \quad \rho\left(x, \gamma_{1} \gamma_{2}\right)=\rho\left(x, \gamma_{1}\right) \rho\left(x \cdot \gamma_{1}, \gamma_{2}\right) .
$$

We recall that (see [35] p. 167) the algebraic hull of $\rho$ in $G$ is the smallest algebraic subgroup $H$ of $G$ (up to conjugacy) such that there exists a Borel map $\varphi: \Omega \rightarrow G$ for which the cocycle $\rho_{\varphi}(x, \gamma)=\varphi^{-1}(x) \rho(x, \gamma) \varphi(x \cdot \gamma)$ takes its values in $H$. Here we consider the case where $\Gamma=\mathbb{Z}$, $X$ is the product space $\Omega=G^{\mathbb{Z}}$ endowed with a product probability. As a consequence of our result, we obtain the algebraic hull of the cocycle associated to the product of coordinates of $\Omega$. More precisely, we obtain the stronger following result.

4.1. THEOREM. - Let $G$ be an algebraic real semi-simple Zariski connected group and $\mu$ be a probability measure on $G$. We assume that the Zariski closure of the closed sub-semigroup $T_{\mu}$ generated by the support of $\mu$ is equal to $G$. We consider the product probability space $\Omega=G^{\mathbb{Z}}$ endowed with the product measure $\mathbb{P}=\mu \otimes \mathbb{Z}$. We denote by $Y_{k}(\omega), k \in \mathbb{Z}$, the coordinates of $\omega \in \Omega$ and by $\theta$ the shift transformation on $\Omega$. Clearly $\theta$ preserves the probability measure $\mathbb{P}$ on $\Omega$ and $\mathbb{P}$ is $\theta$-ergodic. Furthermore, the $G$-valued random variables $Y_{k}, k \geqslant 1$, are independent and identically distributed with common law $\mu$.

We set:

$$
S_{0}=e ; \quad \forall n \in \mathbb{N}^{*}, \quad S_{n}=Y_{0} \cdots Y_{n-1} \quad \text { and } \quad S_{-n}=Y_{-1}^{-1} \cdots Y_{-n}^{-1} .
$$

Then there exists a Borel map $\Phi: \Omega \longrightarrow G$ such that, $\mathbb{P}$-a.e.: $\Phi P^{+}=\widehat{Z} P^{+}($see (3.4)) and

$$
\forall n \in \mathbb{Z}, \quad \Delta_{n}=\Phi^{-1} S_{n} \Phi \circ \theta^{n} \in A M_{T_{\mu}}, \quad \mathbb{P} \text {-a.e. }
$$


Moreover, the subgroup $M_{T_{\mu}} A$ is minimal for this property. Since $M A$ is Zariski-connected, $M A$ is the algebraic hull of the cocycle $\left(S_{n}\right)_{n \in \mathbb{Z}}$.

Proof. - Considering the $F$-valued random variable $Z$ of Theorem 3.4, which depends only on r.v. $\left\{Y_{k}, k \in \mathbb{N}\right\}$, we know that (see $[17,18]$ )

$$
\forall n \in \mathbb{N}, \quad \widehat{Z}^{-1} S_{n} \widehat{Z} \circ \theta^{n} \in M A N^{+}, \quad \mathbb{P} \text {-a.e. }
$$

Let $k$ be a recurrent point on $N^{-} A \backslash G$. The subgroup $M_{T_{\mu}}$ is generated by the elements

$$
\gamma\left(k t k^{-1}, k \cdot x\right)=\gamma(k, t \cdot x) \gamma(t, x) \gamma^{-1}(k, x),
$$

with $t \in T_{\mu}, x \in L_{T_{\mu}} \cap \pi\left(k^{-1} N^{-}\right)$such that $t \widehat{k \cdot x} \in B$. It follows that, the random variables $\gamma(k, Z) \gamma\left(S_{n} \widehat{Z} \circ \theta^{n}\right) \gamma^{-1}\left(k, Z \circ \theta^{n}\right), n \in \mathbb{N}$, belong to $M_{T_{\mu}}$, $\mathbb{P}$-a.e., and

$$
\forall n \in \mathbb{N}, \quad \gamma(k, Z) \widehat{Z}^{-1} S_{n} \widehat{Z} \circ \theta^{n} \gamma^{-1}\left(k, Z \circ \theta^{n}\right) \in A M_{T_{\mu}} N^{+}, \quad \mathbb{P} \text {-a.e. }
$$

Noting that $\forall n \in \mathbb{N}^{*}, S_{-n}=\left(S_{n} \circ \theta^{-n}\right)^{-n}$, these equalities are valid for any $n \in \mathbb{Z}$.

Let $\check{\mu}$ be the image of $\mu$ by the map $g \rightarrow g^{-1}$. In the same way, replacing $\mu$ by $\check{\mu}$ and taking a $\check{\mu}$-recurrent point $\breve{k}$ on $N^{-} A \backslash G$, we obtain, with obvious notations:

$$
\forall n \in \mathbb{N}^{*}, \quad \gamma(\check{k}, \check{Z}) \widehat{\check{Z}}^{-1} S_{-n} \widehat{\check{Z}} \circ \theta^{-n} \gamma^{-1}\left(\check{k}, \check{Z} \circ \theta^{-n}\right) \in M_{T_{\mu}^{-1}} A N^{+}, \quad \mathbb{P} \text {-a.e. }
$$

where $\check{Z}$ is a random variable depending only on the coordinates $\left\{Y_{-k}, k \in \mathbb{N}^{*}\right\}$. As above these equalities are valid for any $n \in \mathbb{Z}$.

We obtain, via the last assertion of Theorem 1.9:

$$
\forall n \in \mathbb{Z}, \quad w \gamma(\check{k}, \check{Z}) \widehat{\check{Z}}^{-1} S_{n} \widehat{\check{Z}} \circ \theta^{n} \gamma^{-1}\left(\check{k}, \check{Z} \circ \theta^{n}\right) w^{-1} \in N^{-} A M_{T_{\mu}}, \quad \mathbb{P} \text {-a.e. }
$$

Now the $N$-valued r.v. $\widehat{Z}$ and $\widehat{Z}$ are independent and, for any $g \in G$ and for $\mathbb{P}$-almost every $\omega, g \widehat{Z(\omega)} \in B$ and $g \widehat{Z}(\omega) \in B$. It follows that, for $\mathbb{P}$-almost every $\omega, w \widehat{\check{Z}}^{-1}(\omega) \widehat{Z}(\omega) \in B$. We introduce the $B$-valued random variable

$$
U=w \gamma(\check{k}, \check{Z}) \widehat{\check{Z}}^{-1} \widehat{Z} \gamma^{-1}(k, Z) .
$$

We have (see (1) and (2))

$$
\begin{aligned}
& \gamma(k, Z) \widehat{Z}^{-1} Y_{0} \widehat{Z} \circ \theta \gamma^{-1}(k, Z \circ \theta) \in A M_{T_{\mu}} N^{+} \text {and } \\
& w \gamma(\check{k}, \check{Z}) \widehat{Z}^{-1} Y_{0} \widehat{Z} \circ \theta \gamma^{-1}(\check{k}, \check{Z} \circ \theta) w^{-1} \in N^{-} A M_{T_{\mu}} .
\end{aligned}
$$

It follows that $\gamma(U, \pi(e)) M_{T_{\mu}}=\gamma(U \circ \theta, \pi(e)) M_{T_{\mu}}$ and the coset $\gamma(U, \pi(e)) A M_{T_{\mu}}$ is $\mathbb{P}$-a.e constant; we denote by $m$ the element of $M$ such that $\gamma(U, \pi(e)) A M_{T_{\mu}}=m A M_{T_{\mu}}, \mathbb{P}$-a.e. Then we can write, $\mathbb{P}$-a.e., $U(\cdot)=u_{-}(\cdot) m \gamma(\cdot) u_{+}(\cdot)$, with $u^{-} \in N^{-}, \gamma \in A M_{T_{\mu}}$ and $u_{+} \in N^{+}$. We set: $\Phi=u_{-}^{-1} w \gamma(\check{k}, \check{Z}) \widehat{\check{Z}}^{-1}=m \gamma u_{+} \gamma(k, Z) \widehat{Z}^{-1}$.

From (1) and (2) we obtain, $\mathbb{P}$-a.e.

$$
\Phi S_{n} \Phi^{-1} \circ \theta^{n} \in\left(A M_{T_{\mu}} N^{+} \cap N^{-} A M_{T_{\mu}}\right)=A M_{T_{\mu}} .
$$

To prove the last assertion, we need the three following lemmas.

$4^{\mathrm{e}}$ SÉRIE - TOME $40-2007-\mathrm{N}^{\circ} 2$ 
4.2. LemMA. - Let $H$ be a co-compact normal subgroup of $M_{T_{\mu}} A$. We call $\lambda$ the normalized Haar measure on the compact group $M_{T_{\mu}} A N^{+} / H N^{+}$. We denote by $\tilde{\theta}$ the transformation of the product space $\Omega \times G / H N^{+}$defined by $\tilde{\theta}(\omega, \xi)=\left(\theta(\omega), Y_{0}^{-1}(\omega) \xi\right)$. Then the measure $m(d \omega, d \xi)=\left(\delta_{\widehat{Z}(\omega)} * \lambda\right)(d \xi) \mathbb{P}(d \omega)$ is an ergodic $\tilde{\theta}$-invariant measure on $\Omega \times G / H N^{+}$.

Proof. - We call $\Pi$ the projection of $\Omega=G^{\mathbb{Z}}$ onto $\Omega_{-}=G^{-\mathbb{N}^{*}} ; \Pi(\omega)=\left(Y_{-k}(\omega)\right)_{k \geqslant 1}$. We set $\widetilde{\Pi}(\omega, \xi)=(\Pi(\omega), \xi)$ and we denote by $\eta$ the transformation of $\Omega_{-} \times G / H N^{+}$defined by $\eta\left(\omega_{-}, \xi\right)=\left(\theta^{-1}\left(\omega_{-}\right), Y_{-1} \xi\right)$. We have $\widetilde{\Pi} \circ \tilde{\theta}^{-1}=\eta \circ \widetilde{\Pi}$ and the probability $\widetilde{\Pi}(m)=\mathbb{P}_{-} \otimes(\nu * \lambda)$ is the $\eta$-invariant, Markov probability on $\Omega_{-} \times G / H N^{+}$defined by $\nu * \lambda$, where $\nu$ is the unique $\mu$-stationary probability on $F$.

From Theorem 2.4, $\sigma=\nu * \lambda$ is an extremal $\mu$-stationary probability on $G / H N^{+}=F \times$ $M A / H$. Then (see [29] Corollaire 1.8) we know that the probability $\widetilde{\Pi}(m)$ is ergodic. Using the ergodic theorem and the approximation of any general Borel function $f$ on $\Omega$ by a Borel function on $\Omega$ depending only on coordinates of index at most $k, k \in \mathbb{N}$, we obtain the convergence of the Birkhoff average of $f$ towards the integral of $f$. Hence the result.

4.3. LemMA. - Let $H$ be a closed subgroup of $M_{T_{\mu}}$ A for which there exists a Borel map $\Psi: \Omega \longrightarrow G$ such that $\forall n \in \mathbb{Z}, \Psi^{-1} S_{n} \Psi \circ \theta^{n} \in H, \mathbb{P}$-a.e. Then there exists $w \in W=M^{\prime} / M$ such that $\widehat{Z} P^{+}=\Phi P^{+}=\Psi w P^{+}$.

Proof. - We set $\xi=\Phi^{-1} \circ \Psi$. We have $\xi H=\Delta_{n} \xi \circ \theta^{n} H$, $\mathbb{P}$-a.e.

For any $w \in W$, since $N^{-} w P^{+}$is $M A$-invariant, we have $\left\{\xi \in N^{-} w P^{+}\right\}=\{\xi \circ \theta \in$ $\left.N^{-} w P^{+}\right\}$; hence $\mathbb{P}\left[\left\{\xi \in N^{-} w P^{+}\right\}\right]=0$ or 1 . As $G=\bigcup_{w \in W} N^{-} m P^{+}$, there exists $m \in W$ such that $\mathbb{P}\left[\left\{\xi \in N^{-} w P^{+}\right\}\right]=1$. Let $V$ be a compact neighborhood of identity element in $N^{-}$ such that $\mathbb{P}\left[\left\{\xi \in V w P^{+}\right\}\right]>0$. We set

$$
\Omega_{0}=\left\{\omega \in \Omega: \forall \alpha \in \Delta_{+}, \lim _{n \rightarrow+\infty} \varphi_{\alpha}\left(\Delta_{n}(\omega)\right)=+\infty\right\} .
$$

From Theorem 3.4, it follows that $\mathbb{P}\left[\Omega_{0}\right]=1$. From the ergodic theorem, for $\mathbb{P}$-a.e. $\omega \in \Omega$, there exists an increasing sequence $(\varphi(n))_{n \geqslant 0}$ of natural integers such that, for any $n \in \mathbb{N}$, $\xi \circ \theta^{\varphi(n)}(\omega) \in V w P^{+}$. It follows that, for $\mathbb{P}$-a.e. $\omega \in \Omega_{0}, \Delta_{\varphi(n)}(\omega) \xi \circ \theta^{\varphi(n)} P^{+}=w P^{+}$and $\xi P^{+}=w P^{+}$.

In the same way there exists $w^{\prime} \in W$ such that, for $\mathbb{P}$-a.e. $\omega \in \Omega_{0}, P^{-} \xi^{-1}=P^{-} w^{\prime}$. One deduces that $w=w^{\prime}$ and, for $\mathbb{P}$-a.e. $\omega \in \Omega_{0}, \xi \in w M A$ and $\Psi w^{-1} P^{+}=\Phi P^{+}$. Hence the result.

Remark. - Under the moment condition $\int_{G} \log \max \left\{\|g\|,\left\|g^{-1}\right\|\right\} \mu(d g)<+\infty$, the Lyapunov exponents of $\Delta_{n}$ are the same as the cocycle $S_{n}$ and $\Phi$ is Lyapunov (see [18]). Moreover the r.v. $\log \|\Phi\|$ is "often" $\mathbb{P}$-integrable (see [30] (4.9)).

4.4. Corollary. - Assume Hypothesis of Lemma 4.3. Let $\left\{c_{i}: 1 \leqslant i \leqslant s\right\}$ be a complete set of coset representatives of the quotient group $M / M_{T \mu}$. Then there exists $i \in\{1, \ldots, s\}$ such that, $\mathbb{P}$-a.e., $\Psi w M_{T_{\mu}} A N^{+}=\widehat{Z} M_{T_{\mu}} A N^{+} c_{i}$.

Proof. - We assume that $w=e$. We set

$$
\Omega_{0}=\left\{\widehat{Z}(\omega) P^{+}=\Psi(\omega) P^{+}\right\} \cap\left\{\Psi^{-1} Y_{0} \Psi \circ \theta \in H\right\} .
$$


$\mathbb{P}\left[\Omega_{0}\right]=1$. For $\omega \in \Omega_{0}$, we have

$$
\Psi(\omega) \in \widehat{Z}(\omega) M A N^{+}=\bigcup_{1 \leqslant i \leqslant s} \widehat{Z}(\omega) M_{T_{\mu}} A N^{+} c_{i} .
$$

So there exists $i(\omega)$ such that $\Psi(\omega) \in \widehat{Z}(\omega) M_{T_{\mu}} A N^{+} c_{i(\omega)}$. Consequently

$$
\Psi(\omega) M_{T_{\mu}} A N^{+}=\widehat{Z}(\omega) M_{T_{\mu}} A N^{+} c_{i(\omega)}
$$

and acting by $Y_{0}^{-1}(\omega)$ we get that the same equality is true for $\theta(\omega)$, hence $i(\theta(\omega))=i(\omega)$. The result follows from ergodicity of $\mathbb{P}$.

4.5. Proof of last assertion of Theorem 4.1. - Let $H$ be a proper closed subgroup of $M_{T_{\mu}} A$ for which there exists a Borel map $\Psi: \Omega \longrightarrow G$ such that $\forall n \in \mathbb{Z}, \Psi^{-1} S_{n} \Psi \circ \theta^{n} \in H$, $\mathbb{P}$-a.e.

(1) If $H[M, M] \neq A M_{T_{\mu}}$, we replace $H$ by a larger proper co-compact and normal subgroup of $M_{T_{\mu}} A$, which contains $[M, M]$. From Lemma 4.3 there exists $w \in W$ such that $\widehat{Z} P^{+}=$ $\Psi w P^{+}$. Replacing $\Psi$ by $\Psi w$ and $H$ by $w^{-1} H w$ we can assume that $\widehat{Z} P^{+}=\Psi P^{+}$. From Corollary 4.4 there exists $c_{i} \in M$ such that $\Psi M_{T_{\mu}} A N^{+}=\widehat{Z} M_{T_{\mu}} A N^{+} c_{i}$, $\mathbb{P}$-a.e.

The measure $\rho(d \omega, d \xi)=\delta_{\Psi(\omega) H N^{+}}(d \xi) \mathbb{P}(d \omega)$ is $\tilde{\theta}$-invariant on $\Omega \times G / H N^{+}$(and obviously ergodic). Moreover any right-translate of $\rho$ by elements of $M_{T_{\mu}} A / H$ is also $\tilde{\theta}$-invariant.

If $\lambda$ is the normalized Haar measure on $M_{T_{\mu}} A / H$, we have

$$
m(d \omega, d \xi)=\delta_{\Psi(\omega)} * \lambda * \delta_{c_{i}^{-1}}(d \xi) \mathbb{P}(d \omega),
$$

which contradicts the ergodicity of $m$ (Lemma 4.2).

(2) If $H[M, M]=A M_{T_{\mu}}$ we first show that we can assume $H \supset A$.

Suppose that $\overline{A H}=M_{T_{\mu}} A$ then, we have

$$
[\overline{A H}, \overline{A H}]=[H, H]=[M, M]
$$

It follows that $[M, M] \subset H$ and $H=A M_{T_{\mu}}$, which is excluded. So we can replace $H$ by $\overline{A H}$.

Now we assume $H \supset A$ and we consider the ergodic measure $m$ on $\Omega \times G / A N^{+}$given by Lemma 4.2:

$$
m(d \omega, d \xi)=\delta_{\widehat{Z}(\omega)} * \lambda_{M_{T_{\mu}}}(d \xi) \mathbb{P}(d \omega)
$$

From the above we know that, on $K$ :

$$
\delta_{\widehat{Z}(\omega)} * \lambda_{M_{T_{\mu}}} * \delta_{c_{i}}=\delta_{\Psi(\omega)} * \lambda_{M_{T_{\mu}}}=\int_{{ }_{H} \backslash^{M_{T_{\mu}} A}} \delta_{\Psi(\omega)} * \lambda_{A \backslash H} * \delta_{x} d x
$$

where $d x$ is the $M_{T_{\mu}} A$-invariant probability measure on ${ }_{H} \backslash^{M_{T_{\mu}} A}$. It follows that

$$
m * \delta_{c_{i}}=\int_{H \backslash^{M_{T_{\mu}} A}} \delta_{\Psi(\omega)} * \lambda_{A \backslash \backslash^{H}} * \delta_{x} d x \mathbb{P}(d \omega)
$$

which contradicts the ergodicity of $m$.

$4^{\mathrm{e}}$ SÉRIE - TOME $40-2007-\mathrm{N}^{\circ} 2$ 


\section{Examples and remarks}

\subsection{A class of compact homogeneous spaces}

In situations of geometric character, isometric extensions often appear in the following set up. Let $X$ be a compact $G$-space, $C$ a compact group which acts freely on $X$. We assume that the two actions commute and we consider the $G$-action (resp. $C$-action) as a left-action (resp. right-action). We assume also that the space $X / C$ of $C$-orbits is a homogeneous space $G / H$ of $G$. We denote by $\tilde{C}$ the inverse image in $X$ of the origin $0 \in G / H$, by $\tilde{0} \in \tilde{C}$ a point of $\tilde{C}$. Then we can identify $\tilde{C}$ with $C$ using the map $c \longrightarrow \tilde{0} \cdot c$. It follows that $\tilde{C}$ is $H$-invariant and for any $h \in H$ we can write $h \cdot \tilde{0}=\tilde{0} \cdot \rho(h)$ where $\rho$ is a homomorphism of $H$ into $C$. Then the map from $G \times C$ to $X$ defined by $(g, c) \longrightarrow g \cdot(\tilde{0} \cdot c)$ allows to identify $G / H \times C$ with $X$. Hence we will write below $X=G / H \times{ }_{\rho} C$. If we take $H=P^{+}$, we have the situation considered in this paper. In this case, we denote again by $\rho$ the restriction of $\rho$ to $A N^{+}$, we write $\overline{\rho(A)}=C_{1}$ and we consider the space $X=G / A N^{+} \times{ }_{\rho} C_{1}=K \times{ }_{\rho} C_{1}$. Then $C$ can be written as $C=(\rho(M) / \rho(M) \cap \overline{\rho(A)}) \times C_{1}$ and $F \times{ }_{\rho} C_{1}$ appears as the space of $\rho(M) \cap \overline{\rho(A)}$-right orbits in $X$. Also the action of $G$ on $K \times{ }_{\rho} C_{1}$ can be written as a skew product:

$$
g \cdot(k, c)=\left(g \cdot k, \rho\left(b^{\prime}(g k)\right) c\right)
$$

Hence, any isometric extension of $F$ by $C$ can be reduced to this special action. In particular, let $D$ be a closed subgroup of $A$ such that $A / D$ is compact, hence $A / D$ is a $q$-dimensional torus $\mathbb{T}^{q}$ with $q \leqslant \operatorname{dim} A$. Then $D N^{+}$is a closed subgroup and the homogeneous space $X=G / D N^{+}$is compact. Also $M A / D$ acts on the right on $X$ and the space of orbits is $G / M A N^{+}=F$. In this case, we have $C=M \times \mathbb{T}^{q}$ and $X=K \times{ }_{\rho} A / D$, where $\rho$ is the canonical homomorphism of $M A$ onto $M A / D$. If $D=A$, we are in the special case $X=G / A N^{+}=K$. Then Theorem 2.4 and its corollary describe the structure of $\mu$-stationary measures and $T$-minimal sets on $K \times \mathbb{T}^{q}$ and $K$.

\subsection{Spheres, shells over $\mathbb{R}, \mathbb{C}, \mathbb{H}$ and products of random matrices}

It is clear that the results of this paper give also the structure of $T$-orbits and of $\mu$-stationary measures for factor spaces of $G / P^{+} \times{ }_{\rho} C$. A family of such situations is given by the compact homogeneous spaces $G / L$ where $L$ is a closed subgroup of $G$ which contains $N^{+}$, since from [34] we know such a space $G / L$ is a factor space of $G / D N^{+}$with $D$ a cocompact subgroup of $M A$. In particular, assume that $V$ is a $n$-dimensional vector space over $\mathbb{F}=\mathbb{R}, \mathbb{C}$ or $\mathbb{H}$, and $G \subset \mathrm{GL}(V)$ is non compact. Assume also that a $K$-invariant scalar product on $V$ is given , denote by $S(V)$ the unit sphere of $V$ and assume $K$ is transitive on $S(V)$. Then $S(V)$ can be considered as a factor space of $G / A N^{+}$, where the action of $g \in G$ on $v \in S(V)$ is given by $g \cdot v=\frac{g v}{\|g v\|}$. We denote by $\mathbf{P}(V)$ the projective space of $V$, by $\mathbf{U}_{\mathbb{F}}$ the compact group of elements of norm 1 in $\mathbb{F}$ and we observe that $S(V)$ is a fiber bundle over $\mathbf{P}(V)$ with typical fiber $\mathbf{U}_{\mathbb{F}}$. We use a natural Borel section (see Lemma 2.2), in order to write $S(V)=\mathbf{P}(V) \times \mathbf{U}_{\mathbb{F}}$. We denote by $\lambda_{\mathbb{F}}$ the normalized Haar measure on $\mathbf{U}_{\mathbb{F}}$, by $m$ the uniform measure on $\mathbb{T}$, by $\nu_{0}$ the unique $\mu$-stationary measure on $\mathbf{P}(V)$. Also, given a number $k>1$, the factor space $S_{k}(V)$ of $V \backslash\{0\}$ by the dilation group $k^{\mathbb{Z}}$ is a compact homogeneous space of $G$ as above. We can write

$$
S_{k}(V)=S(V) \times \mathbb{T}, \quad g \cdot(v, z)=\left(g \cdot v, z\|g v\|^{\frac{2 i \pi}{\ln k}}\right) .
$$

So that $S_{k}(V)$ appears as a "shell" in $V \backslash\{0\}$ with the 2 spherical boundaries identified by the dilation of ratio $k$. We will consider below the unit sphere $S(V)$ as a limiting case of $S_{k}(V)$ 
(with $k=1$ ). Also, using the Borel section above, we write $S_{k}(V)=\mathbf{P}(V) \times \mathbf{U}_{\mathbb{F}} \times \mathbb{T}$ and we decompose the measures accordingly.

If we consider the product of random matrices $\mathrm{七}_{j}(\omega)=Y_{j}(\omega) \cdots Y_{1}(\omega) \in \mathrm{SL}(n, \mathbb{F})$ where $\left(Y_{k}\right)_{k \geqslant 1}$ are i.i.d. random matrices distributed according to $\mu$, with $\int_{G} \ln \|g\| \mu(d g)<+\infty$, the behavior of the length of the column vector $L_{j}(\omega) v(v \in V \backslash\{0\})$ is given by the largest Lyapunov exponent $\lambda(\mu)>0$ (see [10]). But the multiplicity of $\lambda(\mu)$ is equal to $\operatorname{dim}_{\mathbb{R}} \mathbb{F}$ (i.e. 1, 2 or 4). Since the direction of $€_{j}(\omega) v$ is represented by a point of the unit sphere $S(V)$, the theorem gives the behaviour of the "angular" part of $L_{j}(\omega) v$ : the component $\lambda_{\mathbb{F}}$ of the limiting measure $\sigma$ gives its equidistribution on $\mathbf{U}_{\mathbb{F}}=\{ \pm 1\}$ or $\mathbb{T}, \mathbf{S}^{3}$ depending on the multiplicity of $\lambda(\mu)$. See [2] for the problem of "winding" of $L_{k}(\omega) v$. In particular, taking $G=\operatorname{SL}(n, \mathbb{R}), \operatorname{SL}(n, \mathbb{C})$ or $\mathrm{SL}(n, \mathbb{H})$ we get, from Theorem 2.4, its corollary and the spectral decomposition theorem of [29], the following result, which extends results of [11].

COROLlaRY. - Assume that $V=\mathbb{R}^{n}, \mathbb{C}^{n}$ or $\mathbb{H}^{n}, G=\mathrm{SL}(n, \mathbb{R}), \mathrm{SL}(n, \mathbb{C})$ or $\mathrm{SL}(n, \mathbb{H}), T$ is a Zariski dense sub-semigroup of $G, \mu$ is a probability measure on $G$ such that $T_{\mu}=T$ and $k>1$. Then, if $T \subset G$ and in the special case $T \subset \mathrm{SL}(n, \mathbb{R}), T$ does not preserve a convex cone of $\mathbb{R}^{n}$, the action on $S_{k}(V)$ (resp. $S(V)$ ) has a unique minimal set $\Phi_{k}$ (resp. $\left.\Phi\right)$. Furthermore $\mu$ has a unique stationary measure $\sigma_{k}=\nu_{0} \otimes \lambda_{\mathbb{F}} \otimes m$ (resp. $\sigma=\nu_{0} \otimes \lambda_{\mathbb{F}}$ ), the support of $\sigma_{k}$ (resp. $\sigma$ ) is $\Phi_{k}($ resp. $\Phi)$ and for any $x \in S_{k}(V)$ (resp. $S(V)$ ), we have the following weak convergence: $\lim _{n} \mu^{n} * \delta_{x}=\sigma_{k}\left(\right.$ resp. $\left.\lim _{m} \mu^{m} * \delta_{x}=\sigma\right)$. For any $x \in S(V)$ and $\mathbb{P}$-almost every $\omega \in \Omega$, the sequence of probabilities $\frac{1}{m} \sum_{j=0}^{m-1} \delta_{L_{j}(\omega) \cdot x}$ converges to $\nu_{0} \otimes \lambda_{\mathbb{F}}$.

\subsection{An isometric extension of the Farey transformation}

The situation considered in this paper is closely related to the situation of isometric extension of hyperbolic transformations, via the tool of transfer operators (see [12,22]).

In order to illustrate Theorems 2.4 and 2.6, we consider a simple situation related to Farey algorithm. We recall that the mediant of two adjacent irreducible fractions, $\frac{p}{q}$ and $\frac{p^{\prime}}{q^{\prime}}$, is given by $\frac{p+p^{\prime}}{q+q^{\prime}}$ which is also irreducible since $\left|p q^{\prime}-p^{\prime} q\right|=1$. Starting from $\frac{0}{1}$ and $\frac{1}{1}$ and writing the successive mediants give the ordered list of irreducible fractions in $[0,1]$. At stage $n$, one obtains $2^{n}+1$ mediants $\frac{p_{k}}{q_{k}}, 0 \leqslant k \leqslant 2^{n}$, and it is known that the sequence $v_{n}=\frac{1}{2^{n}+1} \sum_{k=0}^{2^{n}} \delta \frac{p_{k}}{q_{k}}$ converges towards the so-called Minkowski measure $\nu$. The measure $\nu$ is singular, purely continuous and $\operatorname{Supp}(\nu)=[0,1]$ (see [33]). We introduce the two projective maps $a$ and $b$ of $[0,1]$ into itself defined by

$$
a(t)=\frac{t}{1+t} \quad \text { and } \quad b(t)=\frac{1}{2-t},
$$

corresponding to the matrices $\left[\begin{array}{ll}1 & 0 \\ 1 & 1\end{array}\right]$ and $\left[\begin{array}{cc}0 & 1 \\ -1 & 2\end{array}\right]$ of $\operatorname{SL}(2, \mathbb{R})$.

We consider the probability measure $\mu$ on $\mathrm{SL}(2, \mathbb{R})$ defined by $\mu=\frac{1}{2}\left(\delta_{a}+\delta_{b}\right)$ and we denote by $P_{\mu}$ the Markov operator on $[0,1]$ defined by $P_{\mu}(t, \cdot)=\frac{1}{2}\left(\delta_{a(t)}+\delta_{b(t)}\right)$. It is easy to see that $P_{\mu}^{n}(1 / 2, \cdot)$ converges weakly to $\nu$, hence $\nu$ is the $\mu$-stationary measure. We observe that $a$ and $b$ are the inverses branches of the map $u$ defined by

$$
u(t)= \begin{cases}\frac{t}{1-t} & \text { if } 0 \leqslant t<\frac{1}{2}, \\ 2-\frac{1}{t} & \text { if } \frac{1}{2} \leqslant t \leqslant 1\end{cases}
$$

and $P_{\mu}$ is the adjoint of $u$ with respect to $\nu$; hence $u(\nu)=\nu$.

$4^{\text {e }}$ SÉRIE - TOME $40-2007-\mathrm{N}^{\circ} 2$ 
We observe that $u$ is topologically conjugate to the map $x \longrightarrow\{2 x\}$ from $\mathbb{T}$ into itself and $\nu$ corresponds to the uniform probability on $\mathbb{T}$. However $u$ is not expanding on $[0,1]$ since $u^{\prime}(0)=1$. Also the above conjugacy is not absolutely continuous.

Furthermore, the cone $\mathcal{C}=\left\{(x, y) \in \mathbb{R}^{2}: 0 \leqslant x \leqslant y\right\}$ is invariant under $a$ and $b$. Its intersection with the line $y=1$ can be identified with the interval $[0,1]$. We denote by $\overline{\mathcal{C}}$ the factor space of $V$ by the dilation group $2^{\mathbb{Z}}=\left\{2^{n}: n \in \mathbb{Z}\right\}$ and we observe that $P_{\mu}$ acts naturally on $\overline{\mathcal{C}}$; this action appears as an isometric extension of $P_{\mu}$ and we denote by $\widetilde{P_{\mu}}$ the corresponding Markov operator. The space $\overline{\mathcal{C}}$ can be visualized as the trapezoid $\mathcal{D} \subset[0,1] \times[1,2]$ defined by

$$
\mathcal{D}=\left\{(x, y) \in \mathbb{R}^{2}: 1 \leqslant y \leqslant 2,0 \leqslant x \leqslant y\right\}
$$

with identification of the horizontal sides. If we denote by $m$ the normalized Haar measure on the torus associated with [1,2], then Theorem 2.6 gives the weak convergence

$$
\lim _{n \rightarrow+\infty} \widetilde{P}_{\mu}^{n}(v, \cdot)=\nu \otimes m=\tilde{\nu} .
$$

We write $\overline{\mathcal{C}}$ in the coordinates

$$
t=\frac{x}{y}, \quad s=\log _{2} y, \quad(t, s) \in[0,1] \times[0,1],
$$

we denote by $\{x\}$ the fractional part of $x \in \mathbb{R}$ and we consider the function $f$ defined by

$$
f(t)= \begin{cases}\log _{2}(1-t) & \text { if } 0 \leqslant t \leqslant 1 / 2 \\ \log _{2} t & \text { if } 1 / 2 \leqslant t \leqslant 1\end{cases}
$$

Then the map $\tilde{u}$ given by $\tilde{u}(t, s)=(u t,\{s+f(t)\})$ is an isometric extension of $u$ by the circle group $\mathbb{T}$. It is easy to see that $\widetilde{P}_{\mu}$ is the adjoint of $\tilde{u}$ with respect to $\tilde{\nu}$ and, for any probability measure $\alpha$ on $\overline{\mathcal{C}}$ absolutely continuous with respect to $\tilde{\nu}$, we have $\lim _{n} \tilde{u}^{n}(\alpha)=\tilde{\nu}$. It follows that $\tilde{u}$ is mixing with respect to $\tilde{\nu}$. The above discussion can be summarized in the following way:

Proposition. - The extension $\tilde{u}$ on $[0,1] \times \mathbb{T}$ of the Farey transformation u given by:

$$
\tilde{u}(t, s)=(u t,\{s+f(t)\})
$$

with

$$
f(t)= \begin{cases}\log _{2}(1-t) & \text { if } 0 \leqslant t \leqslant 1 / 2, \\ \log _{2} t & \text { if } 1 / 2 \leqslant t \leqslant 1\end{cases}
$$

is mixing with respect to $\tilde{\nu}=\nu \times m$ where $\nu$ is the Minkowski measure on $[0,1]$ and $m$ the Haar measure on $\mathbb{T}$.

The operator $P_{\mu}$ on $[0,1]$ has a spectral gap (see $[15,20]$ ) in the space of $\varepsilon$-Hölder functions, for $\varepsilon$ small enough, but it is unclear if the same is true for $\widetilde{P}_{\mu}$. Presumably, from [12], exponential mixing of $\tilde{u}$ and validity of a CLT are true for $\varepsilon$-Hölder functions, but the validity of a local limit theorem is unclear for us. Note that the nonlinearity of the function $t \rightarrow \log _{2}(1-t)$ implies that the present situation differs essentially from the classical situation of a random walk on the circle group. We thank S. Gouezel for relevant information. 


\section{4. $T$-invariant cones in linear representations of $G$}

It is possible to show that for any subgroup $\Phi$ of $M / M_{0}$, there exists a Zariski dense subsemigroup $T$ of $G$ such that $\operatorname{Sgn} T=M_{T} / M_{0}=\Phi$. Here we only show that the two extreme situations $M_{T}=M$ and $M_{T}=M_{0}$ occur and we relate $T$-invariant cones in $\mathbb{R}^{n}$ to minimal subsets of $\mathbf{S}^{n-1}$, in case $G=\mathrm{SL}(n, \mathbb{R})$.

Proposition. - Assume $T$ is a lattice in $G$. Then $M_{T}=M$.

Proof. - It is well known (see [14]) that there exists a probability measure $\mu$ supported on $T$, such that its stationary measure $\nu$ on $F=G / P^{+}$is the $K$-invariant probability measure. Then the Haar measure $\tilde{\nu}$ on $K=G / A N^{+}$is $\mu$-stationary. In view of Theorem 2.4, the proposition will follow from the $T$-ergodicity of $\tilde{\nu}$. This amounts to show that $A N^{+}$is ergodic for the $G$-invariant measure on $T \backslash G$. But this follows from Howe-Moore theorem since $A N^{+}$is unbounded (see [35]).

As a second situation, we consider $G=\mathrm{SL}(n, \mathbb{R})$ and the semigroup $T$ of totally positive matrices (see [21]). We consider the canonical basis $e_{I}$ of $\bigwedge^{p} \mathbb{R}^{n}(1 \leqslant p \leqslant n)$. We recall that a matrix $g \in \operatorname{End}\left(\mathbb{R}^{n}\right)$ is said to be totally positive if, for any $p, \bigwedge^{p} g$ is represented by a positive matrix with respect to $e_{I}$. Also the eigenvalues of such a matrix are positive and the totally positive matrices of $\mathrm{SL}(n, \mathbb{R})$ form a closed semigroup $T$ with non empty interior. It follows that $T$ is Zariski dense in $\operatorname{SL}(n, \mathbb{R})$ and $\langle\operatorname{Spec} T\rangle=] 0,+\infty\left[{ }^{n-1}\right.$. From Theorem 1.9 we conclude $M_{T}=M_{0}$, hence $\operatorname{Sgn} T=\{e\}$.

Proposition. - Assume $T \subset \mathrm{SL}(n, \mathbb{R})$ is the semigroup of totally positive matrices. Then $M_{T}=M_{0}$.

In order to illustrate Theorem 3.16, we consider $G=\operatorname{SL}(2 n, \mathbb{R})$ and the semigroup $T$ of totally positive matrices. Let $\mu$ be a probability measure on $G$ supported by $-T$ such that the Zariski closure of $T_{\mu}$ is equal to $G$. Then $\operatorname{Sgn} T=\{e\}, \operatorname{Sgn} T_{\mu}=\mathbb{Z} / 2 \mathbb{Z}$ and each $T_{\mu}$-invariant minimal subset of $K=G / A N^{+}$splits in two cyclic classes.

More generally non triviality of $M / M_{0}$ is closely related to the existence of non-trivial subsemigroups of $G$ leaving invariant a convex cone in some linear representation of $G$. This property plays also an important role in the classification of divisible convex cones (see [5]). In that paper another proof of the fact that $M_{T}$ has finite index in $M$ is given.

The relation between the number of $T$-minimal subsets on $S(V)$ and $T$-invariant convex cones can be described in an elementary way in case $G=\mathrm{SL}(n, \mathbb{R})$. Clearly an element is $\mathbf{P}^{n-1}$-proximal if it is semi-simple and has a simple real dominant eigenvalue. We say that the semigroup $T \subset G$ satisfies condition $i-p$ (see [19]) if:

$-T$ does not preserve a finite union of projective subspaces;

- there exists in $T$ an element $t$ which is $\mathbf{P}^{n-1}$-proximal.

In terms of Zariski closure it is equivalent to say that the connected component of the Zariski closure of $T$ is irreducible, semi-simple and contains a proximal element (see [26]). Then we have the following result, shown in a different way in [7].

Proposition (See Propositions 3.11 and 3.15 of [7].). - Assume $T \subset \mathrm{SL}(n, \mathbb{R})$ satisfies condition $i-p$. Then we have the dichotomy:

(a) The T-action on $\mathbf{S}^{n-1}$ has a unique minimal set.

(b) The T-action on $\mathbf{S}^{n-1}$ has two minimal sets.

In case (a) the minimal set is symmetric and in case (b) the two minimal sets are symmetric of each others.

Each of the two following conditions is equivalent to (b):

$4^{\text {e }}$ SÉRIE - TOME $40-2007-\mathrm{N}^{\circ} 2$ 
$\left(\mathrm{b}^{\prime}\right)$ T leaves invariant a convex cone with nonempty interior which do not contain a line;

$\left(\mathrm{b}^{\prime \prime}\right)$ the dominant eigenvalues of the $\mathbf{P}^{n-1}$-proximal elements of $T$ are positive.

Proof. - We set $Y=\bigcap_{x \in \mathbf{S}^{n-1}} \overline{T \cdot x}$ and we observe that $Y$ is $T$-invariant symmetric and contained in any $T$-invariant closed subset of $\mathbf{S}^{n-1}$. We recall that $T$ has a unique $T$-minimal set $L_{T}^{1}$ on $\mathbf{P}^{n-1}$ (see [16]). If $Y \neq \emptyset$, then $Y$ is minimal by construction. Since $Y$ is contained in any $T$-minimal set $Z$, we have $Y=Z$.

If $Y \neq \emptyset$, we denote by $x_{0}$ a point of $\mathbf{S}^{n-1}$ which projects on a point of $L_{T}^{1} \subset \mathbf{P}^{n-1}$. We define

$$
Y_{1}=\left\{x \in \mathbf{S}^{n-1}: x_{0} \in \overline{T \cdot x}\right\} .
$$

Then $\mathbf{S}^{n-1}=Y_{1} \cup-Y_{1}$. Let $Y_{2}=\bigcap_{x \in Y_{1}} \overline{T \cdot x}$. Then $x_{0} \in Y_{2}$, hence $Y_{2} \neq \emptyset$.

Furthermore $Y_{2}$ is the unique minimal subset of $Y_{1}$. Also $-Y_{2}$ is the unique minimal subset of $-Y_{1}$.

If $T$ preserves a convex cone as in condition $\left(\mathrm{b}^{\prime}\right)$, then it preserves also its symmetric. This gives the two invariant subsets of $\mathbf{S}^{n-1}$, hence we are in case (b). Conversely, assume (b) and let $\widehat{Y_{0}}$ be the convex cone generated by the minimal set $Y_{0}$; we show that $\widehat{Y_{0}} \cap-\widehat{Y_{0}}=\{0\}$. Otherwise, we can find $y_{1}, \ldots, y_{p} \in Y_{0}, z_{1}, \ldots, z_{q} \in-Y_{0}$ and $\alpha_{i}, \beta_{j}>0,1 \leqslant i \leqslant p, 1 \leqslant j \leqslant q$, such that:

$$
y=\sum_{i=1}^{p} \alpha_{i} y_{i}=\sum_{j=1}^{q} \beta_{j} z_{j}=z .
$$

Then, there exists $t \in T$ with a simple dominant eigenvalue and a dominant eigenvector $v \in Y_{0}$ such that $y_{i}, z_{j}$ do not belong to the $t$-invariant complementary hyperplane of $v$ (see [15]). Then $t^{n} \cdot y$ converges to $v \in Y_{0}$ while $t^{n} \cdot z$ converges to $-v \in Y_{0}$. Since $v \neq-v$, this is a contradiction. Hence $\widehat{Y_{0}}$ is contained in an open half space.

From the Perron-Frobenius theorem, it is clear that the condition $\left(b^{\prime}\right)$ implies condition $\left(b^{\prime \prime}\right)$. In order to prove the converse we need to use some more notations.

We consider the following subgroups of $\operatorname{SL}(n, \mathbb{R})$ :

$$
\begin{aligned}
N_{1}^{-} & =\left\{\left[\begin{array}{cc}
1 & 0 \\
{ }^{t} u & I_{n-1}
\end{array}\right]: u \in \mathbb{R}^{n-1}\right\}, \quad(A M)_{1}=\left\{\operatorname{diag}\left(\lambda^{-(n-1)}, \lambda I_{n-1}\right): \lambda \in \mathbb{R}^{*}\right\}, \\
S_{1} & =\{\operatorname{diag}(1, A): A \in \operatorname{SL}(n-1, \mathbb{R})\} \quad \text { and } \quad N_{1}^{+}=\left\{\left[\begin{array}{cc}
1 & u \\
0 & I_{n-1}
\end{array}\right]: u \in \mathbb{R}^{n-1}\right\} .
\end{aligned}
$$

Then any matrix $g \in \operatorname{SL}(n, \mathbb{R})$ such that $\left\langle e_{1}, g e_{1}\right\rangle \neq 0$ can be written as $g=\eta_{1}^{-} \gamma^{1}(g) s_{1} \eta_{1}^{+}$with $\eta_{1}^{-} \in N_{1}^{-}, \eta_{1}^{+} \in N_{1}^{+}, s_{1} \in S_{1}, \gamma^{1}(g) \in(A M)_{1}$.

We denote by $\langle\operatorname{Spec} T\rangle_{1}$ the closed subgroup of $\mathbb{R}^{*}$ generated by the dominant eigenvalues of the $\mathbf{P}^{n-1}$-proximal elements of $T$. We know that, since $T$ satisfies condition $i-p,\langle\operatorname{Spec} T\rangle_{1}$ is equal to $\mathbb{R}^{*}$ or $\mathbb{R}_{+}^{*}$ (see [19]). We can assume that the direction of $e_{1}$ belongs to $L_{T}^{1}$. Then, as in the proof of Proposition 1.8, we get, for $t \in T, \gamma^{1}(t) \in\langle\operatorname{Spec} T\rangle_{1}$. If $\langle\operatorname{Spec} T\rangle_{1}=\mathbb{R}_{+}^{*}$, then $\gamma^{1}(t)>0$. It follows that $T e_{1} \subset \mathbb{R}^{n}$ is contained in the half space $x_{1} \geqslant 0$. Hence $T$ preserves a convex cone with non zero interior. In others words condition $\left(b^{\prime \prime}\right)$ implies condition $\left(b^{\prime}\right)$.

\subsection{Spectrum of $T$}

It follows from Theorem 1.9 that the projection of $\langle\operatorname{Spec} T\rangle \subset M A /[M, M]$ in $A$ is equal to $A$, a fact also proved in [5]. This fact suffices to prove Theorem 1.9 if $\operatorname{Arg} T$ is finite, i.e. $M_{0}=[M, M]$, in particular if $G=\mathrm{SL}(n, \mathbb{R}), \operatorname{Sp}(n, \mathbb{R}), \mathrm{SO}(p, q), p \neq q+2$. More precisely, the 
$\mathbb{R}$-almost simple classical groups such that $M_{0}=[M, M]$ are locally isomorphic to one of the list [25]:

$$
\mathrm{SL}(n, \mathbb{R}), \operatorname{SO}(p, q)(p \neq q+2), \operatorname{Sp}(n, \mathbb{R}), \mathrm{SL}(n, \mathbb{H}), \mathrm{U}_{2 p}^{*}(\mathbb{H}) \text { and } \operatorname{Sp}(p, q) .
$$

Those for which $M_{0} /[M, M]$ is a non-trivial torus $\mathbb{T}^{s}$ are given by the list:

$$
\mathrm{SL}(n, \mathbb{C}), \mathrm{SU}(p, q), \mathrm{Sp}(n, \mathbb{C}), \mathrm{SO}(n, \mathbb{C}), \mathrm{SO}(p+2, p) \text { and } \mathrm{U}_{2 p+1}^{*}(\mathbb{H})
$$

This follows by inspection of the Satake diagrams in Table 9 of [25], since $s$ is equal to the number of arrows. Furthermore, it follows from [8] that, for simply connected $G$, we have $M / M_{0}=(\mathbb{Z} / 2 \mathbb{Z})^{r^{\prime}}$ where the exponent $r^{\prime}$ is equal to the number of "real roots" of the complexification of $G$. Hence, in general, the group $\langle\operatorname{Spec} T\rangle$ takes into account the pairing of "angular" and "radial" parts of the elements in $T^{\text {prox }}$. For a detailed study of $\langle\operatorname{Spec} T\rangle$ in case of $\operatorname{SL}(2, \mathbb{C})$, see [13]. For the study and use of the group $\langle\operatorname{Spec} T\rangle$ in the context of the mixing properties of frame flows in negative curvature, see [22]. In that paper, for a natural class of $T$ 's, a geometrical proof of the facts that $M_{T}=M=S O(n)$ and $\langle\operatorname{Spec} T\rangle$ projects surjectively on $A=\mathbb{R}$ is given, for the case of $G=\mathrm{SO}(n, 1)$.

If $T$ is Zariski dense, one can expect $T$ to contain enough elements which are proximal and generic with respect to Diophantine properties. Such facts are proved in complement below due to G. Prasad.

In case $G$ has a complex structure, for example $G=\mathrm{SL}(n, \mathbb{C})$, one can obtain more information on the local structure of $L_{T}$. The situation of $\mathrm{SL}(n, \mathbb{C})$ is analyzed in the following complement by S. Cantat.

\section{Complement}

\section{By Serge Cantat}

The goal of this complement is to show that the limit set of a Zariski dense subgroup of the $\mathbb{R}$-algebraic group $\operatorname{SL}(n, \mathbb{C})$ is not locally contained in strict analytic subsets of $\mathbf{P}^{n-1}(\mathbb{C})$. The proof illustrates the use of the main results of [26] or [6] Appendix. It is an easy combination of Chow's theorem concerning analytic subsets of projective varieties (see [24]) and of the existence of $\mathbb{R}$-regular elements in Zariski-dense subgroups [26,28].

\subsection{Notations}

Let $T$ be a sub-semigroup of $\operatorname{SL}(n, \mathbb{C})$. The flag space of $\operatorname{SL}(n, \mathbb{C})$ is the usual flag space $\operatorname{Flag}(n, \mathbb{C})$ of complete flags

$$
\{0\}=V_{0} \subset V_{1} \subset \cdots \subset V_{n-1} \subset V_{n}=\mathbb{C}^{n}
$$

in $\mathbb{C}^{n}$. The Plücker embedding shows that $\operatorname{Flag}(n, \mathbb{C})$ is a smooth projective variety of dimension $n(n-1) / 2$ in a huge projective space $\mathbf{P}\left(\mathbb{C}^{N}\right)$ : The group $\operatorname{SL}(n, \mathbb{C})$ acts linearly on $\mathbb{C}^{N}$ and the induced action on the ambient projective space $\mathbf{P}\left(\mathbb{C}^{N}\right)$ preserves the subvariety $\operatorname{Flag}(n, \mathbb{C})$. When $T$ is Zariski-dense in $\operatorname{SL}(n, \mathbb{C})$, we shall denote by $L_{T} \subset \operatorname{Flag}(n, \mathbb{C})$ the limit set of $T$. Then $L_{T}$ is a closed subset of $\operatorname{Flag}(n, \mathbb{C})$.

$4^{\text {e }}$ SÉRIE - TOME $40-2007-\mathrm{N}^{\circ} 2$ 


\subsection{Complex analytic dimension}

Let $\Lambda$ be a closed subset of a smooth complex analytic space $M$. The complex analytic dimension of $\Lambda$ at a point $p$ is defined by

$$
\operatorname{adim}_{\mathbb{C}}(\Lambda ; p)=\min \left\{\operatorname{dim}_{\mathbb{C}}(W)\right\}
$$

where $W$ describes the set of germs of complex analytic subsets of $M$ that contain $\Lambda$ in a

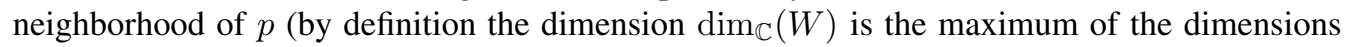
of the irreducible components of $W$ ).

The map $p \mapsto \operatorname{adim}_{\mathbb{C}}(\Lambda ; p)$ is a semi-continuous function from $\Lambda$ to

$$
\left\{0,1,2, \ldots, \operatorname{dim}_{\mathbb{C}}(M)\right\} .
$$

The complex analytic dimension of $\Lambda$ is defined as

$$
\operatorname{adim}_{\mathbb{C}}(\Lambda)=\max _{p \in \Lambda}\left\{\operatorname{adim}_{\mathbb{C}}(\Lambda ; p)\right\}
$$

Remark. - Let $T$ be a sub-semigroup of $\operatorname{SL}(n, \mathbb{C})$. Since $\operatorname{adim}_{\mathbb{C}}\left(L_{T}, p\right)$ is a $T$-invariant and semi-continuous function of $p \in L_{T}$ and $L_{T}$ is $T$-minimal, this function is constant and equal to $\operatorname{adim}_{\mathbb{C}}\left(L_{T}\right)$.

Remark. - Let $\Lambda$ be a closed subset of $M$ of complex analytic dimension $d$. Let $p$ be a point of $\Lambda$. By definition, there exist a neighborhood $\mathcal{U}_{p}$ of $p$ and a complex analytic subset $W$ of $\mathcal{U}_{p}$ of dimension $d$ that contains $\Lambda \cap \mathcal{U}_{p}$. Let $W_{1}$ be an irreducible component of $W$ and let $W_{1}^{\prime}$ be the subset of points $q$ in $W_{1}$ which are not contained in a second irreducible component of $W: W_{1}^{\prime}$ is an open analytic subset of $W_{1}$ and $W$ coincides locally with $W_{1}$ around any point $q$ of $W_{1}^{\prime}$. As a consequence, if $q$ is a point of $W_{1}^{\prime}$ there is a neighborhood $\mathcal{U}_{q}$ of $q$ such that $W_{1}^{\prime}$ contains $\Lambda \cap \mathcal{U}_{q}$. Then

$$
\operatorname{adim}_{\mathbb{C}}(\Lambda ; q) \leqslant \operatorname{dim}_{\mathbb{C}}\left(W_{1}^{\prime}\right)=\operatorname{dim}_{\mathbb{C}}\left(W_{1}\right) \leqslant \operatorname{dim}_{\mathbb{C}}(W) .
$$

When $\Lambda$ is a limit set $L_{T}$, there exists an element $\gamma$ of $T$ such that $\gamma(p)$ is contained in $\mathcal{U}_{q}$. As a consequence, we can replace $W$ by $\gamma^{-1}\left(W_{1}\right)$ and assume that $W$ is irreducible.

\subsection{Statement of the result}

The goal of this complement is to prove the following theorem,

THEOREM. - Let $T$ be a Zariski-dense sub-semigroup of $\mathrm{SL}(n, \mathbb{C})$. Then

$$
\operatorname{adim}_{\mathbb{C}}\left(L_{T}\right)=\operatorname{dim}_{\mathbb{C}}(\operatorname{Flag}(n, \mathbb{C})) .
$$

\subsection{Preliminaries on algebraic geometry}

We now recall two important results which are proved in details in Chapter 4 of Mumford's book [24].

Let $M$ be a smooth compact complex manifold. A closed subset $X \subset M$ is $*$-analytic if $X$ can be decomposed as

$$
X=X^{(r)} \cup X^{(r-1)} \cup \cdots \cup X^{(0)}
$$


where for any $i, X^{(i)}$ is an $i$-dimensional complex sub-manifold of $M$ and

$$
\overline{X^{(i)}} \subset X^{(i)} \cup \cdots \cup X^{(0)} .
$$

If $X^{(r)}$ is not empty, then $r$ is the dimension of $X$.

THEOREM ([24], p. 60). - A closed subset $X$ of a complex manifold $M$ is $*$-analytic if and only if $X$ is a complex analytic subset of $M$.

THEOREM (Chow's theorem, see [24], p. 61). - Any analytic subset of a projective algebraic variety is a projective algebraic variety.

In what follows, these theorems will be used with $\operatorname{Flag}(n, \mathbb{C})$ or $\mathbf{P}^{k}(\mathbb{C})$ in the role of $M$.

\subsection{Preliminaries on linear transformations}

If $\gamma$ is an invertible linear transformation of $\mathbb{C}^{N}$ such that its eigenvalues $\alpha_{1}, \ldots, \alpha_{N}$ satisfy

$$
\left|\alpha_{1}\right|>\left|\alpha_{2}\right|>\cdots>\left|\alpha_{N}\right|
$$

we will say that $\gamma$ is $\mathbb{R}$-regular (this condition is equivalent to proximality as defined in Section 1.3).

Let $\gamma$ be a $\mathbb{R}$-regular element in $\operatorname{GL}(N, \mathbb{C})$. For each eigenvalue $\alpha$ of $\gamma, L(\alpha)$ will denote the corresponding eigenline. The linear transformation $\gamma$ acts on the projective space $\mathbf{P}\left(\mathbb{C}^{N}\right)$ and each line $L(\alpha)$ gives rise to a fixed point of $\gamma$. We will use the following notations:

- The attracting fixed point of $\gamma$ corresponds to the line $L\left(\alpha_{1}\right)$; it will be denoted by $p(\gamma)$.

- The hyperplane of $\mathbf{P}\left(\mathbb{C}^{N}\right)$, that contains the fixed points which correspond to the lines $L\left(\alpha_{i}\right), i>1$, will be denoted by $H(\gamma)$.

- The basin of attraction of $p(\gamma)$ is the complement of $H(\gamma)$ in $\mathbf{P}\left(\mathbb{C}^{N}\right)$.

Let $\mathcal{U} \subset \mathbf{P}\left(\mathbb{C}^{N}\right)$ be a neighborhood of $p(\gamma)$ such that $\gamma^{-1}(\mathcal{U})$ contains strictly $\overline{\mathcal{U}}$. Let $W$ be an analytic subset of $\mathcal{U}$ which is invariant under the action of $\gamma$, by which we mean

$$
\gamma(W) \subset W, \quad \gamma^{-1}(W) \cap \mathcal{U}=W .
$$

Let $B$ be a small open ball centered at $p$, and contained in $\mathcal{U}$. If the radius of $B$ is small enough, and if we replace $\gamma$ by a sufficiently high iterate $\gamma^{r}$, we may assume that

$$
\bar{B} \subset \gamma^{-1}(B) \subset \gamma^{-2}(\bar{B}) \subset \mathcal{U} .
$$

Let $V$ be the intersection of $W$ with the shell $\gamma^{-1}(B) \backslash B$. If we denote by $X$ the union $\bigcup_{m \geqslant 0} \gamma^{-m}(W)$, the $\gamma$-invariance of $W$ implies that $X$ is the disjoint union

$$
X=(W \cap B) \cup V \cup \gamma^{-1}(V) \cup \cdots \cup \gamma^{-m}(V) \cup \cdots .
$$

Inside each open shell $\gamma^{-m}\left(\gamma^{-2}(B) \backslash \bar{B}\right), X$ coincides with the analytic subset $\gamma^{-m}(W \cap$ $\left.\left(\gamma^{-2}(B) \backslash \bar{B}\right)\right)$. Since the increasing union $\bigcup_{m \geqslant 0} \gamma^{-m}(B)$ fills the basin of $p(\gamma)$, this shows that $X$ is an analytic subset of $\mathbf{P}\left(\mathbb{C}^{N}\right) \backslash H(\gamma)$.

\subsection{Dimension 2}

Let us now prove the theorem in the easiest non-trivial case. In this paragraph, we suppose that $n=3$ and we study the action of a sub-semigroup $T \subset \mathrm{SL}(3, \mathbb{C})$ on $\mathbf{P}^{2}(\mathbb{C})$ (instead of the 3 -dimensional flag space $\operatorname{Flag}(3, \mathbb{C})$ ). We keep the notation $L_{T}$ for the limit set of $T$ in $\mathbf{P}^{2}(\mathbb{C})$.

$4^{\text {e }}$ SÉRIE - TOME $40-2007-\mathrm{N}^{\circ} 2$ 
If the complex analytic dimension of $L_{T}$ is 0 , then $L_{T}$ is a discrete and closed subset of $\mathbf{P}^{2}(\mathbb{C})$. As a consequence, $L_{T}$ is a finite $T$-invariant set. This contradicts the Zariski-density of $T$.

Let us now suppose that the complex analytic dimension of $L_{T}$ is 1 . By [26] there exists a $\mathbb{R}$-regular element $\gamma$ in $T$. Its attracting fixed point $p=p(\gamma)$ is contained in $L_{T}$. Let $W$ be a germ of analytic sub-manifold of dimension 1 containing $L_{T}$ in a neighborhood $\mathcal{U}$ of $p$. By the remark at the end of Section 6.2, we can assume that $W$ is irreducible. If $W$ is not $\gamma$-invariant, then $L_{T}$ is locally contained in the locally finite set $\gamma(W) \cap W$ and this contradicts $\operatorname{adim}_{\mathbb{C}}\left(L_{T}\right)=1$. Then, as a germ of analytic subset around $p, W$ is $\gamma$-invariant.

The set

$$
X=\bigcup_{m>0} \gamma^{-m}(W)
$$

is an increasing union of analytic subsets of dimension 1 in $\mathbf{P}^{2}(\mathbb{C})$.

If $W$ is locally contained in one of the two lines joining $p$ to the other fixed points of $\gamma$, then $\bar{X}$ coincides with this line. If not, we can reduce the neighborhood $\mathcal{U}$ and assume that the germ $W$ intersects these two lines only at $p$. Then the closure of $X$ is the union of $X$ and the repulsive fixed point $q$ of $\gamma$. The previous paragraph shows that $X$ is analytic in the complement of the line $H(\gamma)$. This shows that $\bar{X}$ is a $*$-analytic subset of $\mathbf{P}^{2}(\mathbb{C})$ with $X^{(1)}=X$ and $X^{(0)}=q$. Chow's theorem now says that $X$ is in fact an algebraic subset of dimension 1 in $\mathbf{P}^{2}(\mathbb{C})$.

As a consequence, $p$ is contained in an algebraic curve $\bar{X}$ such that $\bar{X} \cap L_{T}$ is a neighborhood of $p$ in $L_{T}$. Let $y$ be a point of $L_{T}$ which is not contained in $H(\gamma)$. Then $y$ is in the basin of attraction of $p$ and $\gamma^{m}(y)$ is in $\mathcal{U}$, if $m$ is large enough. This implies that $y$ is a point of $X$. Consequently, $L_{T}$ is contained in the one dimensional algebraic set $X \cup H(\gamma)$, the Zariski closure of $L_{T}$ is a one dimensional $T$-invariant subset of $\mathbf{P}^{2}(\mathbb{C})$ and this contradicts the Zariski-density of $T$.

Remark. - In the general case below, we reproduce the same argument, but with the help of several $\mathbb{R}$-regular elements $\gamma_{i}$ of $T$.

\subsection{General case}

The flag space $\operatorname{Flag}(n, \mathbb{C})$ is a complex projective subvariety of some $\mathbf{P}\left(\mathbb{C}^{N}\right)$. The group $\operatorname{SL}(n, \mathbb{C})$ acts linearly on this projective space and the action preserves the image of $\operatorname{Flag}(n, \mathbb{C})$. We assume that the complex analytic dimension of the limit set $L_{T}$ inside $\operatorname{Flag}(n, \mathbb{C})$ is smaller than the dimension of $\operatorname{Flag}(n, \mathbb{C})$.

Let $\gamma$ be a $\mathbb{R}$-regular element of $T$. Let $W$ be a germ of irreducible analytic subset of $\operatorname{Flag}(n, \mathbb{C})$ of dimension $\operatorname{adim}_{\mathbb{C}}\left(L_{T}\right)$ containing $L_{T}$ in a neighborhood $\mathcal{U}$ of the attracting fixed point $p(\gamma)$. This set $W$ is $\gamma$-invariant, because otherwise $\gamma(W) \cap W$ is a germ of complex analytic space that contains locally $L_{T}$ around $p$, the dimension of which is strictly less that $\operatorname{adim}_{\mathbb{C}}\left(L_{T}\right)$. As shown in Section 6.5 above, the increasing union

$$
X=\bigcup_{m \geqslant 0} \gamma^{-m}(W)
$$

is an analytic subset of $\mathbf{P}\left(\mathbb{C}^{N}\right) \backslash H(\gamma)$.

Now, since $T$ is Zariski-dense, the set of $\mathbb{R}$-regular elements of $\operatorname{SL}(n, \mathbb{C})$ form a Zariski-dense subset of $\operatorname{SL}(n, \mathbb{C})$ (see [26]), and we can choose $(k+1) \mathbb{R}$-regular elements $\gamma_{0}=\gamma, \gamma_{1}, \ldots, \gamma_{k}$ of $T$ such that:

- The intersection of the hyperplanes $H\left(\gamma_{i}\right), 0 \leqslant i \leqslant k$, is empty. 
- The attracting fixed points $p\left(\gamma_{i}\right)$ are not contained in $H(\gamma)$.

For each index $i$, the point $p\left(\gamma_{i}\right)$ is contained in $L_{T}$; if $m$ is large enough, $\gamma^{m}\left(p\left(\gamma_{i}\right)\right)$ is in $\mathcal{U}$, and therefore in $W$. This shows that, near $p\left(\gamma_{i}\right), X$ is an irreducible analytic set that locally contains $L_{T}$. Since its dimension is $\operatorname{adim}_{\mathbb{C}}\left(L_{T}\right), X$ is locally $\gamma_{i}$-invariant around $p\left(\gamma_{i}\right)$. Let $X_{i}$ be the increasing union

$$
X_{i}=\bigcup_{m \geqslant 0} \gamma_{i}^{-m}\left(W_{i}\right)
$$

where $W_{i}$ denotes the intersection of $X$ with a small neighborhood of $p\left(\gamma_{i}\right)$. The sets $X_{i}$ are analytic in $\mathbf{P}\left(\mathbb{C}^{N}\right) \backslash H\left(\gamma_{i}\right)$ and they coincide with $X$ near $p\left(\gamma_{i}\right)$. By analyticity this local equality propagates, and we get successively

- $X_{i}$ coincides with $X$ on $\mathbf{P}\left(\mathbb{C}^{N}\right) \backslash\left(H(\gamma) \cup H\left(\gamma_{i}\right)\right)(0 \leqslant i \leqslant k)$;

- $X_{i}$ and $X_{j}$ coincide inside $\mathbf{P}\left(\mathbb{C}^{N}\right) \backslash\left(H\left(\gamma_{i}\right) \cup H\left(\gamma_{j}\right)\right)(0 \leqslant i, j \leqslant k)$.

Since $\bigcap_{i=0}^{k} H\left(\gamma_{i}\right)=\emptyset$, we can define a global analytic subset $\bar{X}$ by

$$
\bar{X}=X_{i} \quad \text { in } \mathbf{P}\left(\mathbb{C}^{N}\right) \backslash H\left(\gamma_{i}\right) .
$$

The set $\bar{X}$ is an analytic subset of the projective space and, by Chow's theorem, $\bar{X}$ is an algebraic subvariety of $\operatorname{Flag}(n, \mathbb{C})$ of dimension $\operatorname{adim}_{\mathbb{C}}\left(L_{T}\right)$. Since $L_{T}$ is contained in $W$ around $p(\gamma)$ and is $\gamma$-invariant, this shows that the Zariski-closure of $L_{T}$ is a strict subvariety of $\operatorname{Flag}(n, \mathbb{C})$. Since this subvariety is $T$-invariant and the action of $\operatorname{SL}(n, \mathbb{C})$ on the flag variety is transitive, we obtain a contradiction with the Zariski-density of $T$.

\section{Complement}

\section{By Gopal Prasad}

In this complement we show that, in a Zariski dense sub-semigroup $\Gamma \subset G$, it is possible to find enough $F$-proximal elements with Diophantine properties. This kind of information is used in the study of orbit closures of a sub-semigroup of $\mathrm{M}(n, \mathbb{Z})$ acting on $\mathbb{T}^{n}$ (see [20,23]). The proofs combine some results of [27] and [28] with information from transcendence theory (see [3]).

Let $\mathbf{G}$ be a semi-simple $\mathbb{R}$-algebraic group which is Zariski connected and does not split over $\mathbb{R}, G=\mathbf{G}(\mathbb{R})$ the group of its $\mathbb{R}$-rational points (see [9]). Let $\mathbb{F} \subset \mathbb{R}$ be a finitely generated subfield of $\mathbb{R}$ such that $\mathbf{G}$ is defined over $\mathbb{F}, \Gamma$ a Zariski dense sub-semigroup of $G$. For the results stated below we can always assume that $\Gamma$ is finitely generated and $\mathbb{F} \subset \mathbb{R}$ is chosen so that $\Gamma \subset \mathbf{G}(\mathbb{F})$. We recall some definitions of [28]. Let $\mathbf{G}^{(1)}, \ldots, \mathbf{G}^{(s)}$ be the connected $\mathbb{F}$-simple normal subgroups of $\mathbf{G}, \mathbf{G}_{1}^{a}, \ldots, \mathbf{G}_{r}^{a}$ its connected absolutely simple normal subgroups. The centralizer of $x \in \mathbf{G}$ will be denoted by $Z_{\mathbf{G}}(x)$ and its identity component (in the Zariski topology) will be denoted by $Z_{\mathbf{G}}^{0}(x)$. If $x \in \mathbf{G}$ is semi-simple it is said to be regular if $Z_{\mathbf{G}}^{0}(x)$ is a maximal torus of $\mathbf{G}$. The regular element $x \in \mathbf{G}(\mathbb{F})$ is said to be $\mathbb{F}$-quasi-irreducible anisotropic if the maximal $\mathbb{F}$-torus $Z_{\mathbf{G}}^{0}(x)$ is $\mathbb{F}$-anisotropic and any $\mathbb{F}$-sub-torus of $Z_{\mathbf{G}}^{0}(x)$ is the almost direct product of sub-tori of the form $\mathbf{G}^{(i)} \cap Z_{\mathbf{G}}^{0}(x)$. We say that $x \in \mathbf{G}$ is without components of finite order if, in some decomposition $x=x_{1} \cdots x_{r}, x_{i} \in \mathbf{G}_{i}^{a}$, all the $x_{i}$ 's have infinite order. The semi-simple element $x \in \mathbf{G}$ is said to be $\mathbb{R}$-regular if the number of eigenvalues of $\operatorname{Ad} g$ of modulus one, is minimum. It is easy to see that $x$ is $\mathbb{R}$-regular if and only if it is $F$-proximal in the sense of Section 1.3. We say that the set $\left\{z_{1}, \ldots, z_{m}\right\} \subset \mathbb{C}^{*}$ is multiplicatively independent if the condition $z_{1}^{n_{1}} \cdots z_{m}^{n_{m}}=1\left(n_{i} \in \mathbb{Z}, 1 \leqslant i \leqslant m\right)$ implies $n_{1}=\cdots=n_{m}=0$. Also we call transcendence degree of $\left\{z_{1}, \ldots, z_{m}\right\}$ the transcendence degree over $\mathbb{Q}$ of the field generated by $z_{1}, \ldots, z_{m}$.

$4^{\text {e }}$ SÉRIE - TOME $40-2007-\mathrm{N}^{\circ} 2$ 
For any regular $g$ in $\Gamma$ we fix a non-real and unbounded character $\chi_{g}$ of the maximal torus $Z_{\mathbf{G}}^{0}(g)$. This implies that $\alpha_{g}=\chi_{g} \bar{\chi}_{g}$ and $\beta_{g}=\chi_{g} \bar{\chi}_{g}^{-1}$ are non-trivial characters of the $\mathbb{R}$-torus $Z_{\mathbf{G}}^{0}(g)$. It is shown in [28] (Theorem 2) that the set $\mathfrak{R} \subset \Gamma$ of regular, $\mathbb{R}$-regular, $\mathbb{F}$-quasi irreducible anisotropic elements without components of finite order is Zariski dense in $G$. Furthermore, it follows from Corollary 1 and Proposition 1 of [27] that there exists, for every $n \in \mathbb{N}$, a subset $\mathfrak{R}_{0}=\left\{g_{1}, \ldots, g_{n}\right\} \subset \mathfrak{R}$ such that the $2 n$ numbers $\alpha_{j}=\alpha_{g_{j}}\left(g_{j}\right)$ and $\beta_{j}=\beta_{g_{j}}\left(g_{j}\right), 1 \leqslant j \leqslant n$, are multiplicatively independent.

Let us recall Schanuel's conjecture (see [3] p. 120): If $z_{1}, \ldots, z_{n}$ are $\mathbb{Q}$-linearly independent complex numbers, then the transcendence degree of $\left\{z_{1}, \ldots, z_{n}, e^{z_{1}}, \ldots, e^{z_{n}}\right\}$ is at least $n$. Then we have the following theorem.

THEOREM. - With the above notations, assume the validity of Schanuel's conjecture. Then there exist $n \in \mathbb{N}$ and $g_{1}, \ldots, g_{n} \in \mathfrak{R}$ such that the subgroup generated by the numbers $\chi_{g_{j}}\left(g_{j}\right)$, $1 \leqslant j \leqslant n$, is dense in $\mathbb{C}^{*}$.

Proof. - We denote by $\varphi$ the group homomorphism of $\mathbb{C}^{*}$ onto $\mathbb{R}_{+}^{*} \times \mathbb{T}$, given by $\varphi(z)=$ $(z \bar{z}, z / \bar{z})$. We fix $\mathfrak{R}_{0}=\left\{g_{1}, \ldots, g_{n}\right\} \subset \mathfrak{R}$ as above and we denote by $L$ the subgroup of $\mathbb{C}^{*}$ generated by the numbers $\chi_{g_{j}}\left(g_{j}\right), 1 \leqslant j \leqslant n$. We observe that $\operatorname{ker} \varphi=\{-1,1\}$ and $\varphi(L)=H$ is the subgroup of $\mathbb{R}_{+}^{*} \times \mathbb{T}$ generated by the pairs $\left(\alpha_{j}, \beta_{j}\right), 1 \leqslant j \leqslant n$. We show below that $\bar{H}=\mathbb{R}_{+}^{*} \times \mathbb{T}$. Since $\mathbb{C}^{*}$ is connected and $\operatorname{ker} \varphi=\{-1,1\}$, this will imply that $L$ is dense in $\mathbb{C}^{*}$.

As $\alpha_{1}, \ldots, \alpha_{n}$ are multiplicatively independent positive numbers, it follows that the projection of $H$ in $\mathbb{R}_{+}^{*}$ is dense. Then, if we assume $\bar{H} \neq \mathbb{R}_{+}^{*} \times \mathbb{T}$, the identity component $\bar{H}^{0}$ of $\bar{H}$ is of finite index $s$ and it coincides with the subgroup $\left\{\left(e^{t}, e^{i t u}\right): t \in \mathbb{R}\right\}$ for some $u \in \mathbb{R}$. From the above, we know that the numbers $\alpha_{j}^{s}=e^{t_{j}}$ and $\beta_{j}^{s}=e^{i t_{j} u}, 1 \leqslant j \leqslant n$, are multiplicatively independent. From Schanuel's conjecture we get that the transcendence degree of $\left\{t_{j}, i t_{j} u, e^{t_{j}}, e^{i t_{j} u}: 1 \leqslant j \leqslant n\right\}$ is at least $2 n$. Let $d$ be the transcendence degree of $\mathbb{F}$ over $\mathbb{Q}$. Since $\alpha_{j}^{s}=e^{t_{j}}$ and $\beta_{j}^{s}=e^{i t_{j} u}$ are character values at $g_{j} \in \mathbf{G}(\mathbb{F})$, they belong to the algebraic closure $\overline{\mathbb{F}}$ of $\mathbb{F}$. Hence the transcendence degree of $\left\{t_{j}, i t_{j} u, e^{t_{j}}, e^{i t_{j} u}: 1 \leqslant j \leqslant n\right\}$ is at most $d+n+1$. Now, if $n>d+1$, we have a contradiction with Schanuel's conjecture.

Now, we recall a consequence of the six exponentials theorem of Lang and Ramachandra. If $\alpha, \gamma$ are given algebraic numbers, the equation in algebraic numbers $\beta, \delta: \log \alpha \log \beta=$ $\log \gamma \log \delta$ cannot have two solutions $\left(\beta_{1}, \delta_{1}\right),\left(\beta_{2}, \delta_{2}\right)$ such that all six logarithms are $\mathbb{Q}$-linearly independent. Using this statement we have the following theorem.

THEOREM. - With the above notations, let $\mathbb{F}$ be a real number field, assume that $\mathbf{G}$ is $\mathbb{F}$-defined and $\Gamma \subset \mathbf{G}(\mathbb{F})$. Then there exist $g_{1}, g_{2}, g_{3}$ in $\Gamma$ such that the numbers $\left\{\chi_{g_{j}}\left(g_{j}\right): 1 \leqslant\right.$ $j \leqslant 3\}$ generate a dense subgroup of $\mathbb{C}^{*}$.

Proof. - We follow the proof of the above theorem with $n=3$ and $\mathfrak{R}_{0}=\left\{g_{1}, g_{2}, g_{3}\right\}$. As above if $\bar{H} \neq \mathbb{C}^{*}$, the algebraic numbers $\log \alpha_{j}, \log \beta_{j}, 1 \leqslant j \leqslant 3$, satisfy: $\frac{\log \beta_{j}}{\log \alpha_{j}}=i u$. From the six exponentials theorem, we get that the numbers $\left\{\alpha_{j}, \beta_{j}: 1 \leqslant j \leqslant 3\right\}$ are not multiplicatively independent. This contradicts the choice of $\mathfrak{R}_{0}$.

Remark. - If we assume that $G=\mathbf{G}(\mathbb{R})$ has real rank at least 2 and $\Gamma$ is a sub-semigroup of an irreducible lattice, then the conditions of the theorem above are satisfied. This follows from the arithmeticity theorem of Margulis. More generally if $G$ is not locally isomorphic to $\operatorname{SL}(2, \mathbb{R})$ or $\operatorname{SL}(2, \mathbb{C})$ and $\Gamma$ is contained in an irreducible lattice, then the conditions of the above theorem are satisfied. 


\section{Acknowledgements}

We thank G. Prasad for useful comments and information on some aspects of this paper. We are grateful to the referee for a careful reading of the manuscript.

\section{REFERENCES}

[1] Abels H., Margulis G.A., Soifer G.A., Semigroups containing proximal linear maps, Israel J. Math. 91 (1995) 1-30.

[2] ARnold L., Random Dynamical Systems, Springer Monographs in Mathematics, Springer-Verlag, Berlin, 1998.

[3] BAKER A., Transcendental Number Theory, Cambridge Univ. Press, Cambridge, UK, 1975, x+147 pp.

[4] Benoist Y., Propriétés asymptotiques des groupes linéaires II, in: Analysis on Homogeneous Spaces and Representation Theory of Lie Groups, Okayama-Kyoto, 1997, in: Adv. Stud. Pure Math., vol. 26, Math. Soc. Japan, Tokyo, 2000, pp. 33-48.

[5] Benoist Y., Convexes divisibles III, Annales Scient. É.N.S. 38 (5) (2005) 793-832.

[6] Benoist Y., LABourie F., Sur les difféomorphismes d'Anosov affines à feuilletages stable et instable différentiables, Invent. Math. 111 (1993) 285-308.

[7] Benoist Y., Automorphismes des cones convexes, Invent. Math. 141 (2000) 149-193.

[8] Borel A., Introduction aux groupes arithmétiques, Hermann, Paris, 1969.

[9] Borel A., Linear Algebraic Groups, second ed., G.T.M., vol. 126, Springer-Verlag, New York, 1996.

[10] Bougerol P., LACroiX J., Products of Random Matrices and Applications to Schrödinger Operators with Random Potential, in: Progress in Probability Theory and Math. Statistics, vol. 8, Birkhäuser, Boston, 1985.

[11] Conze J.P., GuivarC'H Y., Densité d'orbites d'actions de groupes linéaires et propriétés d'équidistribution de marches aléatoires, in: Burger M., Iozzi A. (Eds.), Rigidity in Dynamics and Geometry, Cambridge, 2000, Springer-Verlag, Berlin, 2002, 39-76.

[12] DOLGOPYAT D., On mixing properties of compact group extensions of hyperbolic systems, Israel J. Math. 130 (2002) 157-2005.

[13] FERTE D., Flot horosphérique des repères sur les variétés hyperboliques de dimension 3 et spectre des groupes kleiniens, Bull. Brazilian Math. Soc. 33 (1) (2002) 99-123.

[14] Furstenberg H., Boundary theory and stochastic processes on homogeneous spaces, in: Moore C.C. (Ed.), Harmonic Analysis on Homogeneous Spaces, in: Proc. Symp. Pure Math., vol. 26, Amer. Math. Soc., Providence, RI, 1972, pp. 193-229.

[15] Goldsheid I., Guivarc'H Y., Zariski Closure and the dimension of the Gaussian law of the product of random matrices I, P.T.R.F. 105 (1996) 109-142.

[16] GuivarC'H Y., Produits de matrices aléatoires et applications aux propriétés géométriques des sousgroupes du groupe linéaire, Ergodic Theory Dynam. Systems 10 (1990) 129-131.

[17] GuivarC'H Y., RAugi A., Frontière de Furstenberg, propriétés de contraction et théorèmes de convergence, Z. Wahrsch. Verw. Gebiete 69 (2) (1985) 187-242.

[18] Guivarc'H Y., Raugi A., Contraction properties of an invertible matrix semigroup, Lyapunov coefficients of a product of independent random matrices, Israel J. Math. 65 (2) (1989) 165-196.

[19] GuivarC'H Y., Renewal theorems, product of random matrices, and toral endomorphisms, in: Potential Theory in Matsue, in: Adv. Stud. Pure Math. Math. Soc. Japan, vol. 44, 2006, pp. 5366.

[20] Guivarc'H Y., STARKov A., Orbits of linear group actions, random walks on homogeneous spaces and toral automorphisms, Ergodic Theory Dynam. Systems 24 (2004) 767-802.

[21] KARLIN S., Total Positivity, vol. I, Stanford Univ. Press, Stanford, CA, 1968.

[22] Ledrappier F., Pollicott M., Ergodic properties of linear actions of $2 \times 2$ matrices, Duke Math. $J .116$ (2) (2003) 353-388.

[23] Margulis G.A., Problems and conjectures in rigidity theory, in: Mathematics Frontiers and Perspectives, Amer. Math. Soc., Providence, RI, 2000, pp. 161-174.

$4^{\text {e }}$ SÉRIE - TOME $40-2007-\mathrm{N}^{\circ} 2$ 
[24] Mumford D., Algebraic Geometry, Complex Projective Varieties, Grundlehren Der Mathematischen Wissenschaften, vol. 221, Springer-Verlag, Berlin/New York, 1976.

[25] Onischik A.L., Vinberg E.B., Lie Groups and Algebraic Groups, Springer-Verlag, Berlin/New York, 1990.

[26] PRASAD G., $\mathbb{R}$-regular elements in Zariski-dense subgroups, Quart. J. Math. Oxford, 245 (180) (1994) 541-545.

[27] PRASAD G., RAPINCHUK A., Zariski-dense subgroups and transcendental number theory, Math. Res. Lett. 12 (2-3) (2005) 239-249.

[28] PRASAD G., RAPINCHUK A., Existence of irreducible $\mathbb{R}$-regular elements in Zariski-dense subgroups, Math. Res. Lett. 10 (2003) 21-32.

[29] Raugi A., Théorie spectrale d'un opérateur de transition sur un espace métrique compact, Ann. Inst. H. Poincaré 28 (2) (1992) 281-309. (Fascicule de probabilités, 21 pp., Publ. Inst. Rech. Math. Rennes, 1994, Univ. Rennes I, Rennes, 1994).

[30] RAUGi A., Théorème ergodique multiplicatif, Produits de matrices aléatoires indépendantes, Fascicule de probabilités, 43 pp., Publ. Inst. Rech. Math. Rennes, 1996/1997.

[31] RaUgi A., Fonctions harmoniques et théorèmes limites pour les marches aléatoires sur les groupes, Bull. Soc. Math. France 54 (1977) 5-118.

[32] Rosenblatt M., Equicontinuous Markov operators, Teor. Verojatnost. i Primenen. 9 (1964) 205222.

[33] SALEM R., On some singular monotonic function which are strictly increasing, TAMS 53 (1943) 427439.

[34] WiTte D., Co-compact subgroups of semi-simple Lie groups, in: Benkart G.M., Osborn J.M. (Eds.), Lie Algebras and Related Topics, in: Contemp. Math., vol. 110, 1990, pp. 309-313.

[35] ZIMMER R., Ergodic Theory and Semi-Simple Lie Groups, Monographs in Math., vol. 81, BirkhäuserVerlag, Basel, 1984.

Yves GuIVARC'H

Université de Rennes I, IRMAR,

Campus de Beaulieu,

35042 Rennes Cedex, France

E-mail: yves.guivarch@math.univ-rennes1.fr

Albert RAUGI

Université de Rennes I, IRMAR,

Campus de Beaulieu,

35042 Rennes Cedex, France

E-mail: Albert.Raugi@univ-rennes1.fr 